\title{
Nuclear Export of Drosophila PERIOD contributes to temperature compensation of the circadian clock
}

Astrid Giesecke ${ }^{1}$, Peter S Johnstone ${ }^{2}$, Angelique Lamaze ${ }^{1}$, Johannes Landskron³ , Ezgi Atay ${ }^{1}$, KoFan Chen ${ }^{4}$, Deniz Top ${ }^{2}$, and Ralf Stanewsky ${ }^{1, *}$

${ }^{1}$ Institute of Neuro- and Behavioral Biology, Westfälische Wilhelms University, 48149 Münster, Germany

2Department of Pediatrics (Division of Medical Genetics) and Department of Pharmacolology, Dalhousie University, Halifax, Nova Scotia, Canada B3H 4R2

${ }^{3}$ Centre for Molecular Medicine Norway, University of Oslo, 0318 Oslo, Norway

${ }^{4}$ Department of Genetics and Genome Biology, University of Leicester, Leicester LE1 7RH, United Kingdom

${ }^{*}$ Corresponding author and Lead Contact

Ralf Stanewsky

e-mail: stanewsky@uni-muenster.de 


\section{Abstract:}

Circadian clocks are self-sustained molecular oscillators controlling daily changes of behavioral activity and physiology. For functional reliability and precision the frequency of these molecular oscillations must be stable at different environmental temperatures, known as 'temperature compensation'. Despite being an intrinsic property of all circadian clocks, this phenomenon is not well understood at the molecular level. Here we use behavioral and molecular approaches to characterize a novel mutation in the period (per) clock gene of Drosophila melanogaster, which alters a predicted nuclear export sequence (NES) of the PER protein. We show that this new per ${ }^{1530 A}$ allele leads to progressively longer behavioral periods and clock oscillations with increasing temperature in both clock neurons and peripheral clock cells. While the mutant PER ${ }^{1530 A}$ protein shows normal circadian fluctuations and posttranslational modifications at cool temperatures, increasing temperatures lead to both, severe amplitude dampening and hypophosphorylation of PER ${ }^{1530 \mathrm{~A}}$. We further show that PER $^{1530 A}$ displays reduced repressor activity at warmer temperatures, presumably because it cannot inactivate the transcription factor CLOCK (CLK). With increasing temperatures nuclear accumulation of $P E R^{1530 A}$ within clock neurons is increased, suggesting that PER is normally exported out of the nucleus at warm temperatures. Consequently, downregulating the nuclear export factor CRM1 also leads to temperature-dependent changes of behavioral rhythms. In summary, our results suggest that the PER NES and the nuclear export of clock proteins play an important role in temperature compensation of the Drosophila circadian clock. 


\section{Introduction:}

Circadian clocks allow organisms to anticipate the daily changes of their environment, such as daily fluctuations of light intensity and temperature. They enable animals to restrict their behavioral and physiological activities to species-specific advantageous times of day and night, increasing their overall fitness. Circadian clocks are composed of negative molecular feedback loops, within which clock gene products oscillate in abundance and subcellular localization with a 24-h period (1). In Drosophila melanogaster, expression of the clock genes per and timeless (tim) is mediated by the transcription factors CLK and CYCLE (CYC). PER and TIM proteins accumulate in the cytoplasm before they translocate to the nucleus, bind to the CLK/CYC dimer and repress their own transcription. Eventually, PER and TIM proteins are degraded, allowing CLK and CYC to start a new round of the cycle (1). Posttranslational modifications regulate this process to maintain the period length of the molecular oscillations at 24 h. For example, cytoplasmic PER and TIM phosphorylation by the kinases DBT, CK2 and SGG determines timed nuclear translocation (2). In addition, hyperphosphorylated nuclear PER coincides with hyperphosphorylated, inactive CLK, and the DOUBLETIME (DBT) kinase mediates phosphorylation of both proteins within the same complex leading to transcriptional repression $(3,4)$. In the so called PER 'phosphotimer' the NLK kinase NEMO initially phosphorylates PER S596, which stimulates phosphorylation of S589, S585 and T583 by the CK1 $\varepsilon$ kinase DOUBLETIME (DBT). All these residues belong to the per short phosphocluster, and their phospho-occupancy somehow delays DBT-dependent PER phosphorylation at other residues, most importantly PER S47 (5). Since phosphorylation at PER S47 enables binding of the F-box protein SLIMB and subsequent degradation of PER (6), these consecutive phosphorylation events regulate temporal PER stability and thereby period length of the circadian clock (5). Based on the involvement kinases and phosphatases and other post- 
translational modifications in clock regulation it could be expected that increasing temperature leads to faster enzyme kinetics and therefore shorter periods of molecular oscillations and vice versa. However, circadian clocks are temperature compensated, meaning periods of circadian clock oscillations do not change across a wide range of temperatures (e.g., between $\sim 15^{\circ} \mathrm{C}$ and $29^{\circ} \mathrm{C}$ in fruit flies) (7). Although temperature compensation is a hallmark of all circadian clocks $(8,9)$, its molecular etiology is still not well understood. Circadian clocks can be synchronized by temperature cycles, which suggests that they are temperatureresponsive with compensatory mechanisms (10). This compensation is traditionally explained with the assumption that temperature-mediated changes of different rates across the circadian clock cancel each other out (11). This model is less likely, given that any mutation in circadian genes would yield a temperature-sensitive circadian clock.

Temperature compensation could also be achieved by considering the circadian clock as two coupled oscillators with complementary temperature dependence of period length (10), or as one oscillator composed of biochemical reactions with opposite temperature coefficients (12). This would require different clocks within one organism to have distinct regulatory mechanisms (13). The circadian clock of Drosophila consists of multiple coupled oscillators that vary in their intrinsic period length (e.g. (14)), but it is not known how they behave at different temperatures. Subsets of these oscillators, such as the so-called small lateral neurons (s-LNv), sustain free-running behavioral rhythms in constants darkness (DD) (15), while others such as the Dorsal Neurons 1 - 3 (DN1, DN2, and DN3) are unable, despite sustaining molecular rhythms in DD $(16,17)$. It is therefore conceivable that the different clock neuronal subsets also differ in their ability to maintain temperature-compensated molecular oscillations.

Another possible mechanism for temperature compensation, is that each step in the clock is individually temperature compensated (18). For example, timed nuclear accumulation and 
timed nuclear degradation of PER/TIM would each be temperature compensated. Some alleles of per and tim that are defective in nuclear accululation of the PER/TIM complex also affect temperature compensation. per flies (19) exhibit an increase of behavioral period by four hours from $18^{\circ} \mathrm{C}(27 \mathrm{~h})$ to $29^{\circ} \mathrm{C}(31 \mathrm{~h})$, which correlates well with the $\sim$-hour delay of $\operatorname{PER}^{\mathrm{L}}$ nuclear entry $(20,21)$. This delay can be explained by the weaker interaction of $P E R^{L}$ with TIM with increasing temperatures, because TIM stabilizes PER and PER therefore accumulates even slower at higher temperatures (22).

Similarly, the timblind allele increases behavioral period by four hours in a temperaturedependent manner from $18^{\circ} \mathrm{C}(24 \mathrm{~h})$ to $29^{\circ} \mathrm{C}(28 \mathrm{~h})(23)$. This, and several other new tim alleles map to nuclear export signals (NESs) throughout TIM and also show similar temperature compensation defects (23). Indeed, TIM and PER are subject to active Cargo Protein and Chromosome Maintenance 1 (CRM1)-mediated nuclear export in Drosophila larvae (24). With TIM export, this mechanism relies on CKII-dependent phosphorylation of residue S1404 near a putative NES, which reduces the interaction between TIM and CRM1, and retains normal nuclear levels of TIM and regulation of CLK activity (3). In mammals, mPER1, MPER2, and mPER3 proteins also contain canonical leucine-rich NES motifs, which in cell culture shuttle PER proteins from the nucleus to the cytoplasm in order to target them for degradation $(25,26)$, but their roles in temperature compensation have not been examined.

While it seems clear from these studies that nuclear export of clock proteins plays a role in circadian clock function in both mammals and fruit flies, its role in temperature compensation has not been examined. Here, we generated a point mutation (per $1530 A)$ in the single leucinerich putative PER NES, which is highly conserved to the mPER NES (Figure $1 A)(25,27,28)$ to systematically study the role of the PER NES in the context of temperature compensation. per ${ }^{1530 A}$ mutant flies show substantial temperature-dependent increase of their behavioral 
period, which is correlates well with longer molecular oscillations in several subsets of clock neurons and in peripheral clock cells. PER ${ }^{1530 A}$ shows temperature-dependent alterations of posttranscriptional modifications, accompanied by reduced ability to function as a transcriptional repressor of CLK. Finally, enhanced nuclear PER ${ }^{1530 A}$ accumulation with increasing temperatures, as well as temperature-dependent period lengthening of flies with reduced CRM1 expression strongly implicate nuclear export of PER as an important mechanism for temperature compensation in Drosophila. The knockdown of CRM1 weakens the observed temperature-compensation defect of per ${ }^{1530 A}$, suggesting that nuclear export of other clock proteins may also be affected, and implicating nuclear export as a key mechanism in establishing temperature compensation.

\section{Results:}

\section{The per ${ }^{1530 A}$ mutant causes circadian period lengthening with increasing temperatures}

To analyze the function of the nuclear export signal (NES) in the Drosophila Period (PER) protein, we introduced a single amino acid change into the NES by replacing the conserved isoleucine at position 530 with alanine (PER $\left.{ }^{1530 A}\right)$. To do this, the endogenous per gene was replaced with the mutated per ${ }^{1530 A}$ allele by CRISPR/Cas9 mediated homologous recombination (Figure $1 \mathrm{~A}$ and Methods). To aid detection of wild type and mutated PER ${ }^{1530 \mathrm{~A}}$ we also generated flies where both proteins are endogenously tagged with the V5 epitope (per ${ }^{V 5}$ and peris30A-V5 respectively, Figure $1 \mathrm{~A}$ and Methods). To investigate if the per ${ }^{1530 \mathrm{~A}}$ allele influences circadian clock function and behavioral rhythms, we analyzed locomotor activity in constant darkness at $25^{\circ} \mathrm{C}$ (DD25). While $y w$ control flies showed the typical robust activity rhythms with period values $(\tau)$ of $23.5 \mathrm{hr}$, $\tau$ values of the perlis30A flies were $>2 \mathrm{hr}$ longer $(25.9$ 
hr) (Figure 1B, C, Table 1). Similarly, per ${ }^{v 5}$ flies have $\tau$ values of $23.2 \mathrm{hr}$ compared to $26.7 \mathrm{hr}$ in per $^{1530 A-V 5}$ flies (Figure 1B, C, Table 1). To test if the PER NES is also important for temperature compensation, we tested per ${ }^{1530 A}$ flies at different temperatures (DD18 and DD29). Indeed, per $1530 \mathrm{~A}$ flies shortened their period by $\sim 3 \mathrm{hr}$ to $23.0 \mathrm{hr}$ at $18^{\circ} \mathrm{C}$ and lengthened it to $27.9 \mathrm{hr}$ at $29^{\circ} \mathrm{C}(\mathrm{Q} 10=0.84)$, while $y w$ controls showed almost no change (Q10=0.99) (Figure $1 \mathrm{~B}, \mathrm{C}$, Table 1). Similar results were obtained with the V5-tagged flies: while per $^{V 5}$ flies exhibit stable $\tau$ values around $23 \mathrm{hr}$ from $18^{\circ} \mathrm{C}$ to $29^{\circ} \mathrm{C}(\mathrm{Q} 10=1.0)$, the per $1530 \mathrm{~A}-\mathrm{V} 5$ flies increase $\tau$ from $23.5 \mathrm{hr}$ at $18^{\circ} \mathrm{C}$ to $29.4 \mathrm{hr}$ at $29^{\circ} \mathrm{C}(\mathrm{Q} 10=0.82)$ (Figure $1 \mathrm{~B}, \mathrm{C}$, Table 1$)$. The results unequivocally show that specific mutation of the PER NES leads to a temperature-dependent lengthening of $\tau$, pointing to temperature over-compensation of the circadian clock.

The per ${ }^{1530 A}$ mutation leads to temperature dependent molecular period lengthening within central clock neurons and peripheral clock cells

Behavioral rhythms in constant darkness are driven by molecular oscillations of clock gene expression within circadian pacemaker neurons in the fly brain. It is therefore expected that temperature-dependent period-lengthening also occurs in clock neurons of per ${ }^{1530 A}$ mutant flies. To test this, and to see if temperature compensation is affected equally in all clock neurons, we applied a novel, cell-type restricted real-time luciferase assay (Locally Activatable Bioluminescence or $L A B L)$. Central to $L A B L$ is a period-luciferase transgene, in which /uciferase expression is compromised by a mCherry reporter gene followed by stop codons. This cassette is inserted between the period promoter and the luciferase coding sequences, and flanked by FRT recombination sites (Figure S1A). period-driven luciferase expression can therefore be induced and restricted to subsets of per expressing cells using Gal4 driven expression of UAS- 
FLP (Figure S1A). Details of this technique are described in an accompanying paper (Johnstone et al 2021, this issue). Using LABL we first compared period-luciferase expression in different sets of clock neurons using the Pdf-gal4 (PDF positive s-LNv and I-LNv), DvPdf-gal4 (PDF positive s-LNv and I-LNv, $5^{\text {th }} \mathrm{s}-\mathrm{LNv}, 4 \mathrm{LNd}$ ) and R18H11-gal4 (subset of the DN1p) at three different temperatures in wild type flies. Bioluminescence of 15 male flies per genotype housed in a customized arena was measured using the LumiCycle 32 Luminometer (Actymterics) for 9 days in constant darkness at either $18^{\circ} \mathrm{C}, 25^{\circ} \mathrm{C}$, or $29^{\circ} \mathrm{C}$ (see Materiala and Methods and Johnstone et al 2021, this issue, for details). In wild type flies, all driver lines generated robust bioluminescence oscillations with stable period lengths close to $24 \mathrm{hr}$ at all temperatures (Figure 2A, C, S1B), indicating that all subsets of clock neurons are temperature compensated (cf. (29)). In contrast, the same analysis in the background of the peri530A mutation revealed a significant linear period-lengthening from $18^{\circ} \mathrm{C}$ to $29^{\circ} \mathrm{C}$ for $P d f$ and R18H11-gal4 driver lines and between $18^{\circ} \mathrm{C}$ and $25^{\circ} \mathrm{C}$ for DvPdf-gal4 (Figure 2A, C, S1B) (for unknown reasons the genotype involving per ${ }^{1530 A}$ and $D v P d f$-gal4 died at $29^{\circ} \mathrm{C}$ ). At the lower temperature, bioluminescence oscillations showed a period close to $24 \mathrm{hr}$, while at $25^{\circ} \mathrm{C}$ it was lengthened from $26-28 \mathrm{hr}$, and further to $30-32 \mathrm{hr}$ at $29^{\circ} \mathrm{C}$, depending on the Gal4 driver used to activate LABL (Figure 2D, S1B). Therefore, the period length of the molecular oscillations and behavioral rhythms are equally affected by the per ${ }^{1530 A}$ mutation, showing normal values at $18^{\circ} \mathrm{C}$ and temperature-dependent period lengthening. As a control, we also analyzed period-luciferase expression in $\mathrm{per}^{L}$ flies, which have a period of $27.1 \mathrm{hr}$ at $18^{\circ} \mathrm{C}$, increasing to $29.6 \mathrm{hr}$ at $25^{\circ} \mathrm{C}$ and $31.4 \mathrm{hr}$ at $29^{\circ} \mathrm{C}(20)$. Again, using $P d f$-gal4 to drive $\mathrm{LABL}$, the molecular oscillations closely matched the behavioral ones, with periods of $27-28 \mathrm{hr}$ at $18^{\circ} \mathrm{C}$ lengthening to $29-30 \mathrm{hr}$ at $25^{\circ} \mathrm{C}$, and $32 \mathrm{hr}$ at $29^{\circ} \mathrm{C}$ (Figure S1C). We conclude that LABL is suitable for 
reliable period estimation within clock neurons at different temperatures (see also accompanying paper by Johnstone et al).

To determine whether temperature-dependent defects of the per ${ }^{1530 A}$ allele also extend to peripheral clocks, we next used LABL to measure period-luciferase expression in all clock cells. For this, we applied the tim-UAS-Gal4 driver expressed in all clock cells (30) Johnstone et al 2021, this issue). Although the tim-UAS-Gal4 driver will also activate period-luciferase expression in clock neurons, the vast majority of bioluminescence expression emanates from peripheral clock cells in abdominal tissues and in the head (Johnstone et al 2021, this issue), as previously shown for period-luciferase transgenes expressed in all clock cells (31). As expected, the bioluminescence levels emanating from tim-UAS-Gal4 LABL flies were drastically (40-50 fold) increased compared to the three clock neuronal drivers, reflecting expression in peripheral clock cells (Figure 2B). Similar to the results obtained for the clock neurons, oscillations in peripheral clock cells in wild type flies were temperaturecompensated, with stable periods close to $24 \mathrm{hr}$ at all three temperatures (Figure 2B, C, S1B). Moreover, when analyzed in a er $^{1530 A}$ and $p e r^{L}$ mutant background, tim-UAS-Gal4 driven period-luciferase oscillations showed the same temperature-dependent period-lengthening as observed with the neuronal drivers (Figure 2B, C, S1B, C). Therefore, the per ${ }^{1530 A}$ mutation has a similar effect on both central and peripheral clocks, indicating a common mechanism of temperature compensation.

The per ${ }^{1530 A}$ mutation affects PER and TIM stability and post-translational modification at higher temperatures during LD 
The temperature-dependent period-lengthening of per ${ }^{1530 A}$ flies could be due to decreased $\mathrm{PER}^{1530 \mathrm{~A}}$ stability at higher temperatures. To test this, we performed Western blots of total head protein extracts of control $\left(p^{1 / 5}\right)$ and $p e r^{1530 A-V 5}$ flies raised at different temperatures and collected every six hours during one day in LD (ZT2, ZT8, ZT14, ZT20). To see how wild type PER levels behave at different temperatures throughout the day we first analyzed $p e r^{V 5}$ flies at $18^{\circ} \mathrm{C}, 25^{\circ} \mathrm{C}$ and $29^{\circ} \mathrm{C}$ (Figure 3 ). As expected, we observed robust PER oscillations at all three temperatures, with trough levels at ZT8 and peak expression from ZT20 to ZT2 (Figure 3A) (32). At $18^{\circ} \mathrm{C}$ PER levels appear somewhat reduced from ZT2 to ZT14 compared to the higher temperatures, but the differences were not significant (Figure $3 \mathrm{~A}$ ). We also did not detect obvious differences in the migration speed of PER, which is indicative of its phosphorylation status (33), except that at ZT2 during $29^{\circ} \mathrm{C}$ PER migrates faster compared to the cooler temperatures and therefore appears hypophosphorylated. These results show that wild type PER levels as well as rhythmic changes in abundance and phosphorylation are well compensated against changes of ambient temperature.

Next we compared PER and PER ${ }^{1530 \mathrm{~A}}$ expression at different temperatures. At $18^{\circ} \mathrm{C}$ no obvious differences in protein amounts or mobility could be observed during the four time points we analyzed (Figure $3 \mathrm{~B}$, top panel). In contrast, at $25^{\circ} \mathrm{C}$ an amplitude reduction of $\mathrm{PER}^{1530 \mathrm{~A}}$ cycling due to increased trough levels at ZT8 was apparent (Figure 3B, middle panel). In addition, there was a striking effect on mobility at ZT2 and ZT8. At these time point wild type PER appears mainly in its slow migrating form, which is not observable for PER ${ }^{1530 A}$. Instead the faster migrating form is still present at ZT8, indicating higher stability of PER ${ }^{1530 A}$ due to incomplete post-translational modifications and degradation. This could ultimately result in the lower-amplitude protein cycling we observed (Figure 3B, middle panel). Strikingly, at $29^{\circ} \mathrm{C}$ $\mathrm{PER}^{1530 \mathrm{~A}}$ oscillations were completely abolished and presumably hypophosphorylated protein 
was present at peak levels at all time points analyzed (Figure 3B, lower panel). To summarize, in parallel to the behavioral and luciferase observations, PER ${ }^{1530 \mathrm{~A}}$ protein behaves normal at $18^{\circ} \mathrm{C}$, but both, protein cycling and the phosphorylation pattern become increasingly aberrant with rising temperature. Our results suggest that a temperature-dependent phosphorylation defect underlies the increased stability and reduced oscillation of the PER ${ }^{1530 A}$ protein at higher temperatures.

Next, we examined if the per ${ }^{1530 A}$ mutation had similar effects on the clock protein TIM. First, we again checked if TIM expression varied in wild type in a temperature-dependent manner. We did not detect any differences in levels or migration properties, except for the expected detection of the shorter, cold-induced TIM-SC protein at $18^{\circ} \mathrm{C}$ (Figure $3 \mathrm{~A}$, lower panel) $(34,35)$. As expected from the results with PER, wild type TIM levels and phosphorylation patterns are therefore well compensated against temperature changes. Comparison of TIM between wild type and per ${ }^{1530 A}$ mutants revealed only mild differences at the warmer temperatures, while at $18^{\circ} \mathrm{C}$ they were essentially identical at all time points (Figure S2). At $25^{\circ} \mathrm{C}$ TIM levels in per ${ }^{1530 A}$ were slightly increased at ZT2 and ZT8, and this effect was further pronounced at $29^{\circ} \mathrm{C}$ (Extended Data Figure 3). Nevertheless, even though the amplitude is reduced, TIM levels still oscillated. We were not able to detect any differences in TIM migration between wild type and per $1530 \mathrm{~A}$, but phosphorylation-dependent migration differences are generally not as pronounced for TIM compared to PER (compare Figure 2A, B with S2).

The per ${ }^{1530 A}$ mutation affects PER and TIM stability and post-translational modification at higher temperatures during DD 
Because the temperature-dependent effects on behavioral period length are necessarily determined in constant darkness, we wanted to analyze the effects of the per ${ }^{1530 \mathrm{~A}}$ allele on PER and TIM expression also in DD. PER and TIM isolated from total fly heads mainly reflect expression in the photoreceptor cells, which contain peripheral clocks that dampen out rapidly in DD (16). We therefore analyzed PER and TIM levels during the first day in DD after LD entrainment from both wild type and per ${ }^{1530 A}$ flies at different temperatures. At $18^{\circ} \mathrm{C}$, both PER and TIM cycled with similar amplitude in mutants and controls, although both proteins appeared more stable in per ${ }^{1530 \mathrm{~A}}$ (Figure S3). At $25^{\circ} \mathrm{C}$ and $29^{\circ} \mathrm{C}$ weak PER and TIM oscillations were observed in wild-type, whereas in the per ${ }^{1530 A}$ mutants both proteins were at high constitutive levels (Figure S3). Similar as in LD, PER ${ }^{1530 A}$ protein showed normal daily migration changes at $18^{\circ} \mathrm{C}$, while at $25^{\circ} \mathrm{C}$ and $29^{\circ} \mathrm{C}$ the slow migrating, hyperphosphorylated forms that normally occur in the subjective morning (CT2 and CT8) were absent and instead high levels of faster migrating hypophosphorylated PER ${ }^{1530 A}$ were visible (Figure S3).

In order to track the effects of per ${ }^{1530 A}$ on PER oscillations with high temporal resolution, we turned to the BG-luc reporter, a period-luciferase transgene encoding for $\sim 2 / 3$ rds of the PER protein. This per transgene does not rescue behavioral and molecular arrhythmicity induced by the per ${ }^{01}$ allele, but it accurately reports PER protein cycling in a wild-type background (31), and is therefore suitable to determine the effects of per alleles on PER protein expression. Bioluminescence rhythms emanating from individual adult male $B G$-luc flies were measured for four days in LD followed by three days in DD at the three different temperatures. Strikingly, at $18^{\circ} \mathrm{C}$ BG-luc oscillations in control and per ${ }^{1530 A}$ were of similar amplitude in both LD and DD conditions, with somewhat increased overall expression levels in the mutant (Figure 4A). At $25^{\circ} \mathrm{C} \mathrm{BG-luc}$ oscillations were strongly reduced in the per ${ }^{1530 \mathrm{~A}}$ mutant background compared to the controls, and this effect was further enhanced at $29^{\circ} \mathrm{C}$ (Figure $4 \mathrm{~A}$ ). Oscillations in a wild 
type background were similarly robust at $18^{\circ} \mathrm{C}$ and $25^{\circ} \mathrm{C}$, but amplitude was reduced at $29^{\circ} \mathrm{C}$. Next we analyzed the expression of a tim-luciferase (ptim-TIM-LUC) reporter gene that accurately reflects temporal TIM expression in peripheral clock cells $(29,36)$. In a wild type background, ptim-TIM-LUC oscillations were indistinguishable at $18^{\circ} \mathrm{C}$ and $25^{\circ} \mathrm{C}$, while oscillations were reduced in amplitude at $29^{\circ} \mathrm{C}$ (Figure S4A), similar to what we observed for BG-luc (Figure 4A). This was particularly the case in DD at $29^{\circ} \mathrm{C}$, presumably because oscillations of this peripheral clock reporter dampen rapidly in DD even at lower temperatures (Figure S4A) (36). In the per $1530 \mathrm{~A}$ mutant background, ptim-TIM-LUC oscillations were unaffected at $18^{\circ} \mathrm{C}$, except for a reduced amplitude in DD (Figure S4A). At $25^{\circ} \mathrm{C}$ already in LD a strong reduction of TIM amplitude caused by increased trough levels was observed (Figure S4A). In fact, the remaining oscillations are comparable in amplitude to those observed in clock less per ${ }^{01}$; ptim-TIM-LUC flies at $25^{\circ} \mathrm{C}$, indicating that they are mainly driven by lightdependent TIM degradation during the day (Figure S4B) (37). At $29^{\circ} \mathrm{C}$, the mutants' effects on ptim-TIM-LUC expression are further enhanced, leading to largely arrhythmic expression during LD and DD (Figure S4A). In summary, the luciferase reporter experiments confirm that the per ${ }^{1530 A}$ mutant causes a temperature-dependent impairment of clock protein expression, ranging from normal function at $18^{\circ} \mathrm{C}$ to severe impairments at $29^{\circ} \mathrm{C}$.

Mutations in the per ${ }^{\text {Short }}$ phosphocluster do not cause temperature-dependent period lengthening.

Our Western blot results indicate a temperature-dependent phosphorylation defect caused by per ${ }^{1530 A}$. Because the isoleucine at position 530 is not a target for phosphorylation by a known PER kinase, we investigated if per ${ }^{1530 A}$ may affect PER phosphorylation by some of the 
known PER kinases. To this end, we analyzed mutations affecting the PER 'phosphotimer' known to set clock speed in Drosophila (5). As expected, mutation of the S596 NEMO site $\left(\right.$ per $\left.{ }^{5596 \mathrm{~A}}\right)$ resulted in a drastic period shortening at $25^{\circ} \mathrm{C}(16.5 \mathrm{hr}$, Table 2$)$, but periods were similarly short at both $18^{\circ} \mathrm{C}(16.9 \mathrm{hr})$ and $29^{\circ} \mathrm{C}(15.8 \mathrm{hr})$. Similarly, mutation of a single DBT target site in the per ${ }^{\text {Short }}$ cluster $\left(p e r^{5585 A}\right)$ resulted at short periods at all three temperatures, with an overall $1-\mathrm{hr}$ period shortening at $29^{\circ} \mathrm{C}$ compared to $18^{\circ} \mathrm{C}$ (Table 2). Mutating all 4 phosphorylation sites within the pershort cluster (per $\left.{ }^{T S 583-596}\right)$ also shortened period at all temperatures with a $1 \mathrm{hr}$ increase in clock speed at $29^{\circ} \mathrm{C}$ compared to $18^{\circ} \mathrm{C}$ (Table 2). Finally, mutation of S47A led to the expected period lengthening at $25^{\circ} \mathrm{C}(29.8 \mathrm{hr}$, Chiu et al 2008), but also at $18^{\circ} \mathrm{C}(29.3 \mathrm{hr})$ and $29^{\circ} \mathrm{C}(30.3 \mathrm{hr})$ (Table 2$)$. While these results do not rule out a contribution of the PER phosphotimer in temperature compensation (i.e., mutations within the per $r^{\text {Short }}$ cluster show a tendency to increase clock speed with rising temperature), it seems clear that disruption of this timer does not underlie the drastic overcompensation phenotype of per ${ }^{1530 A}$.

\section{period and timeless transcription is enhanced in per ${ }^{1530 A}$ mutants}

Hyperphosphorylated PER is required for the repression of CLK/CYC mediated per and tim transcription $(4,38,39)$. To test if the lack of fully hyperphosporylated PER in per ${ }^{1530 A}$ flies at $25^{\circ} \mathrm{C}$ and $29^{\circ} \mathrm{C}$ is associated with reduced repressor function we analyzed per and tim transcription using appropriate per-luc and tim-luc reporters $(40,41)$. Bioluminescence emanating from whole flies was recorded as described above in LD and DD conditions at three different temperatures. At $18^{\circ} \mathrm{C}$, both control and per ${ }^{1530 A}$ flies showed robust oscillations of per-luc expression in LD conditions, which rapidly dampen out in DD (Figure 4B). However, 
expression levels in the per ${ }^{1530 A}$ mutants were about 2.5 times higher compared to the controls, indicating reduced repressor or enhanced transcriptional activation function of the mutant protein at this temperature (Figure 4B, C). On average, levels of per transcription in the per ${ }^{1530 A}$ mutant were somewhat reduced compared to those in a per ${ }^{01}$ background (Figure $4 \mathrm{~B}, \mathrm{C})$. At $25^{\circ} \mathrm{C}$ the amplitude of per-luc oscillations in the per ${ }^{1530 \mathrm{~A}}$ mutants was reduced compared to the controls (Figure 4B). Moreover, expression levels were now higher compared to those in the complete absence of PER, in agreement with an enhanced impairment of PER ${ }^{1530 \mathrm{~A}}$ function at $25^{\circ} \mathrm{C}$ compared to $18^{\circ} \mathrm{C}$. The effect on per-luc expression was further enhanced at $29^{\circ} \mathrm{C}$, indicated by significantly higher expression levels compared to those in $\operatorname{per}^{01}$ (Figure 4B, C). Furthermore and like in $p e r^{01}$, expression at $29^{\circ} \mathrm{C}$ was increased during the light phase of the LD cycle in contrast to the clock-dependent per-luc peak occurring during the dark phase (Figure 4B) (40). The most likely explanation for this light-dependent peak in $\operatorname{per}^{01}$ and $p e r^{1530 A}$ flies is the release of per transcriptional repression by TIM, caused by lightdependent degradation of TIM, which also occurs in flies without functional PER (see above) (37). We obtained similar effects of the per ${ }^{1530 A}$ mutation using the tim-luc transcriptional reporter, although at $29^{\circ} \mathrm{C}$ there was no difference in tim transcription between $p e r^{01}$ and per $1530 \mathrm{~A}$ flies (Figure 4C, S4C). In summary, while at $18^{\circ} \mathrm{C}$ both genes are rhythmically transcribed in per ${ }^{1530 A}$ mutants, expression becomes progressively disturbed with increasing temperature. In addition, the high levels of per-luc and tim-luc expression point to an impairment of PER ${ }^{1530 A}$ repressor function and/or gain of transcriptional activator function, particularly pronounced at $25^{\circ} \mathrm{C}$ and $29^{\circ} \mathrm{C}$.

PER:TIM heterodimerization is not grossly altered in per ${ }^{1530 A}$ flies 
A possible reason for reduced repressor function of the $P E R^{1530 A}$ could be the lack or reduction of heterodimerization with TIM. To test if PER:TIM heterodimerization is affected in per ${ }^{1530 A}$ flies, we performed Co-immunoprecipitation (CoIP) experiments using per ${ }^{1530 A-v 5}$ and per $^{15}$ control flies. Head protein extracts were precipitated with anti-V5 antibodies, and Western blots were subsequently probed with anti-TIM antibodies (Figure 5A, Methods). CoIPs were performed at ZT16, a time when PER and TIM are primarily cytoplasmic, and at ZT22, when both proteins are primarily nuclear (42). To compare dimer formation between mutants and controls in a quantitative manner, we normalized bound TIM to the input and represented it as relative signal intensity. This allows to compare relative binding of TIM to wild type and mutant PER within one temperature. Note that with method, it is not possible to compare absolute levels between temperatures (see Materials and Methods). At $18^{\circ} \mathrm{C}$, robust PER:TIM and PER ${ }^{150 A}:$ TIM interactions were observed at both time points, potentially somewhat reduced in the presence of the mutant protein, particularly at ZT16 (Figure 5A). Similar results were obtained at $25^{\circ} \mathrm{C}$ and $29^{\circ} \mathrm{C}$, again showing a trend for reduced PER ${ }^{1530 A}: T_{I M}$ interactions at ZT16 (Figure 5A). Interestingly, at ZT22 this trend was reversed, with higher levels of PER ${ }^{1530 \mathrm{~A}}$ :TIM dimers at $25^{\circ} \mathrm{C}$ and $29^{\circ} \mathrm{C}$ compared to PER:TIM heterodimers at these temperatures and also compared to PER ${ }^{1530 \mathrm{~A}}: \mathrm{TIM}_{\text {dimers at }} 18^{\circ} \mathrm{C}$ (Figure $5 \mathrm{~A}$ ). The results clearly show that the PER ${ }^{1530 A}$ protein is able to form heterodimers with TIM at all temperatures tested, both at ZT16 and ZT22. Faulty PER:TIM heterodimerization is therefore unlikely to explain to observed behavioral and molecular phenotypes of per $1530 \mathrm{~A}$ flies.

\section{PER:PER homodimerization is not grossly affected in per ${ }^{1530 A}$ flies}


In addition to PER:TIM heterodimerization, PER:PER homodimerization is important for PER nuclear entry and PER repressor function (43). We therefore tested if PER ${ }^{1530 A}$ is able to form homodimers at different temperatures. For this, we added HA or MYC antigen tags to a full length per transgene containing the per ${ }^{1530 A}$ mutation and crossed these transgenes into a per $^{01}$ background. Wild type versions of these constructs $\left(p e r^{c-m y c}\right.$ and $p e r^{H A}$ ) restore robust behavioral rhythms in $p e r^{01}$ flies at $25^{\circ} \mathrm{C}$ these rhythms are also temperature compensated (43) (Table S1). As expected per ${ }^{01}$ flies expressing the tagged PER ${ }^{1530 A-M Y C}$ and PER ${ }^{1530 A-H A}$ constructs lengthen their free-running period with increasing temperature, similar to the endogenous per ${ }^{1530 A}$ CRISPR mutants (Table S1). Using Co-IP experiments we have previously shown the abundant existence of PER ${ }^{\mathrm{HA}}$ :PER ${ }^{\mathrm{MYC}}$ dimers in fly heads at $25^{\circ} \mathrm{C}(43)$. Here we show that PER homodimers also form at $18^{\circ} \mathrm{C}$ and $29^{\circ} \mathrm{C}$ (Figure 5B). Next, we compared PER:PER and PER ${ }^{1530 A}: P^{1530 A}$ dimer formation at the different temperatures in the tagged flies at ZT22 (Figure 5B). The results were very similar to those obtained for PER:TIM heterodimerization: While PER 1530A:PER ${ }^{1530 A}$ homodimers do form at all three temperatures, they appear more abundant at $25^{\circ} \mathrm{C}$ and $29^{\circ} \mathrm{C}$ compared to PER:PER homodimers, and this trend is again reversed at $18^{\circ} \mathrm{C}$ (Figure 5B). Overall, the Co-IP experiments suggest that at ZT22 levels of both PER ${ }^{1530 A}: P^{2} R^{1530 A}$ homodimers, as well as PER ${ }^{1530 A}: T_{I M}$ heterodimers are increased compared to the wild type dimers at $25^{\circ} \mathrm{C}$ and $29^{\circ} \mathrm{C}$. Increased levels of nuclear PER ${ }^{1530 \mathrm{~A}}$ caused by faulty nuclear export offers a potential explanation for this observation.

Phosphorylation of the transcription factor CLOCK is altered in per ${ }^{1530 A}$ mutants.

An important function of PER in the nucleus is to bind to the transcription factor CLK, thereby recruiting kinases that phosphorylate and thereby inactivate CLK, resulting in repression of 
per and tim transcription. To see, if the increase per and tim transcription levels in per ${ }^{1530 A}$ flies are caused by inefficient inactivation of CLK, we compared CLK levels and phosphorylation status at ZT2, a time when CLK is maximally phosphorylated and repressed in wild type flies (reference). Strikingly, in per ${ }^{1530 A}$ flies, CLK phosphorylation was severely affected and mainly hypophosphorylated forms were visible at $25^{\circ} \mathrm{C}$ and CLK was barely detectable at $29^{\circ} \mathrm{C}$ (Figure $5 \mathrm{C}$, compare blue and orange arrows in middle panel). Indeed, at $\mathrm{ZT} 2$ and $25^{\circ} \mathrm{C} \mathrm{PER}{ }^{1530 \mathrm{~A}}$ migrated indistinguishably from wild type PER at ZT14, a time when CLK is transcriptionally active and hypophosporylated (Figure 5C, orange arrow) $(4,38)$. In contrast, although CLK levels were reduced in per $r^{1530 \mathrm{~A}}$ mutants at $18^{\circ} \mathrm{C}, \mathrm{CLK}$ migrated similar in wild type and per ${ }^{1530 \mathrm{~A}}$ mutants, indicating normal phosphorylation and repression of CLK (Figure 5C, black bar). Although PER ${ }^{1530 A}$ can form PER:TIM and PER:PER dimers, it appears that its ability to repress CLK (or even binding to CLK) seems to be affected in a temperature dependent manner.

\section{Subcellular localization of $\mathrm{PER}^{1530 \mathrm{~A}}$ is altered at warmer temperatures}

If the per ${ }^{1530 A}$ mutation alters nuclear export in a temperature-dependent way, it should be possible to detect differences in $\mathrm{PER}^{1530 \mathrm{~A}}$ subcellular localization at different temperatures. To see if this the case, we first looked at wild type PER expression in the small ventral lateral neurons (sLNv), which are pacemaker neurons important for the maintenance of behavioral rhythms and for determining the period length in DD (15). Brains of wild type flies were dissected at two time points: ZT16, when PER is largely hypophosphorylated and cytoplasmic, and ZT22, when PER is largely hyperphosphorylated and nuclear. We found that the levels and subcellular localization (ratio of nuclear versus cytoplasmic signals) of PER were similar at $18^{\circ} \mathrm{C}$, and $29^{\circ} \mathrm{C}$ with expected cytoplasmic and nuclear redistribution between ZT16 and ZT22 
(Figure 6A, C, S5). This is in-line with the Western blot results (Figure 3A) and the temperature compensated behavioral period length (Figure 1, Table 1). In contrast, PER ${ }^{1530 A}$ showed temperature-dependent variations in nuclear/cytoplasmic distribution. Compared to wild type, nuclear PER ${ }^{1530 A}$ levels were drastically reduced at $\mathrm{ZT} 22$ and $29^{\circ} \mathrm{C}$ (Figure S5). Strikingly, at ZT16 and the same high temperature, PER ${ }^{1530 A}$ was predominantly nuclear, despite overall lower PER levels (Figure 6, S5). These results indicate that at $29^{\circ} \mathrm{C}$, wild type PER enters the nucleus earlier ( $\leq \mathrm{ZT} 16)$ compared to cooler temperatures ( ZT18) and is then exported again to mitigate premature nuclear accumulation and period shortening at warmer temperatures. We have no explanation for why we observed high $\mathrm{PER}^{1530 \mathrm{~A}}$ at $29^{\circ} \mathrm{C}$ in head extracts, but low levels in the sLNv. One possibility is that in the sLNv premature, nuclear-trapped PER ${ }^{1530 A}$, is rapidly degraded, presumably due to aberrant phosphorylation. In contrast, in the photoreceptor cells, which do not contain a self-sustained molecular clock, clearance of premature-nuclear PER ${ }^{1530 A}$ may not occur, because it may not be required in peripheral clock cells.

\section{The nuclear export factor CRM1/Embargoed contributes to temperature compensation}

If PER is transported by the general nuclear export machinery mediated by CRM1/Embargoed, knock down of embargoed (emb) should also affect temperature compensation. To test this, we used four independent emb RNAi lines to knock down emb expression in all clock cells (timgal4), all clock neurons (Clk856-gal4), or the subset of the ventral lateral clock neurons (Pdfgal4). To increase the efficiency of RNAi knock-down, UAS-dicer was co-expressed with all RNAi constructs (44). While control flies containing only the gal4 or RNAi construct showed stable locomotor activity periods at $18^{\circ} \mathrm{C}, 25^{\circ} \mathrm{C}$, and $29^{\circ} \mathrm{C}$, this was not the case for lines 
expressing emb RNAi constructs in clock cells and clock neurons (Figure 7A, B, Table 3). Strikingly, and similar to per ${ }^{1530 A}$ mutants, knock down of emb led to significantly longer periods at $25^{\circ} \mathrm{C}$ compared to $18^{\circ} \mathrm{C}$. Depending on the driver and the RNAi line the period lengthening varied between $0.7 \mathrm{~h}$ and $5 \mathrm{~h}$ (average $2.8 \mathrm{~h}$ ), indicating a massive disturbance of temperature compensation (Figure 7A, B, Table 3). Interestingly, further increasing the temperature to $29^{\circ} \mathrm{C}$ reversed the effect on clock speed, with periods shortening between 0.5 $\mathrm{h}$ and $2.6 \mathrm{hr}$ (average $1.5 \mathrm{~h}$ ), but not shortening back to the values observed at $18^{\circ} \mathrm{C}$ (Figure 7 $A, B$, Table 3). While this effect on temperature compensation is different to the linear increase of period length in per $1530 \mathrm{~A}$ mutants with temperature, it nevertheless confirms the involvement of nuclear export in temperature compensation. Moreover, it rules out that the observed period lengthening at $25^{\circ} \mathrm{C}$ is simply caused by increased knock down efficiency due to the temperature dependency of the Gal4 system. The period shortening observed at $29^{\circ} \mathrm{C}$ most likely indicates that other clock proteins are also subject to temperature dependent nuclear export. To directly test the interaction between specific knockdown of nuclear export and the per ${ }^{1530 A}$ mutation, we analyzed the behavior of flies expressing emb-RNAi in the per ${ }^{1530 A}$ background (Figure 7C, Table 4). Because we could not co-express UAS-dicer as in the RNAi experiments described above (both UAS-dicer and per ${ }^{1530 A}$ are located on the $X$ chromosome), we compared the period length of the double mutants to those of peri530A single mutants carrying the ga/4 only. Using Pdf-gal4 to drive two different emb-RNAi constructs in per ${ }^{1530 A}$ mutant background resulted in a significant blunting of the temperaturedependent period increase observed in per ${ }^{1530 A}$ single mutants (Figure 7C, Table 4). Moreover, at $25^{\circ} \mathrm{C}$ expression of emb-RNAi BL34021 resulted on $100 \%$ arrhythmic flies, which is not

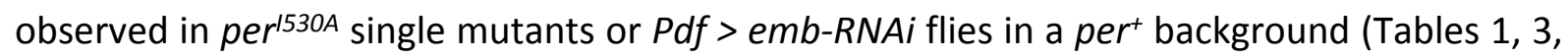
4). Using tim-gal4 to drive emb-RNAi BL31353 in a per ${ }^{1530 A}$ mutant background also blunted 
temperature-dependent period increase compared to per ${ }^{1530 \mathrm{~A}}$, tim-gal4 flies (Figure 7C, Table 4). Taken together, these results indicate an interaction between nuclear export and the per $^{1530 A}$ mutant and suggest that in addition to PER, nuclear export of other clock proteins is regulated in a temperature-dependent manner (see Discussion).

\section{Discussion}

Circadian clocks are able to compensate for the acceleration of biological reactions associated with an increase in temperature. An impairment of temperature compensation should therefore result in a period shortening with increasing temperature. The per ${ }^{1530 \mathrm{~A}}$ mutation has the opposite effect, indicating that this mutation leads to an overcompensation phenotype. Nevertheless, the molecular defects associated with per ${ }^{1530 \mathrm{~A}}$ could shed light on the mechanism underlying temperature compensation, and indeed our findings indicate that nuclear export plays an important role in this process. First, nuclear levels of both PER ${ }^{1530 A}$ :TIM heterodimers and PER ${ }^{1530 \mathrm{~A}}: \mathrm{PER}^{1530 \mathrm{~A}}$ homodimer levels appear increased in per ${ }^{1530 \mathrm{~A}}$ mutants compared to controls, which could be explained by reduced nuclear export efficiency of the mutant protein (Figure 5A, B). Second, subcellular localization of PER ${ }^{1530 A}$ revealed a temperature-dependent atypical nuclear enrichment at $\mathrm{ZT} 16$ at $29^{\circ} \mathrm{C}$, in agreement with the idea that wild type PER is exported from the nucleus at this temperature to mitigate early nuclear accumulation. Finally, knockdown of the nuclear export factor CRM1 effected temperature compensation in opposite ways. In the range from $18^{\circ} \mathrm{C}$ to $25^{\circ} \mathrm{C} \mathrm{emb-RNAi}$ expression resulted in a significant lengthening of period with the same magnitude (about 3$4 \mathrm{~h}$ ) as observed in per $1530 \mathrm{~A}$ flies (Figure 7B, Table 3). In contrast, further increase of temperature to $29^{\circ} \mathrm{C}$ lead to a significant period-shortening by $\sim 1.5 \mathrm{~h}$. These results 
unequivocally show that nuclear export is involved in the temperature compensation mechanism. Moreover, they indicate that in addition to PER, other clock proteins are subject to nuclear export. At low temperatures $\left(18^{\circ} \mathrm{C}\right)$ nuclear export does not seem to play a role in adjusting period length, as both per ${ }^{1530 A}$ and emb-RNAi flies show periods in the normal $24 \mathrm{~h}$ range. At $25^{\circ} \mathrm{C}$, both per ${ }^{1530 A}$ and emb-RNAi flies show essentially the same period-lengthening phenotype, indicating that in wild type flies, PER needs to be exported out of the nucleus in order to maintain a normal period length. At $29^{\circ} \mathrm{C}$ per $r^{150 \mathrm{~A}}$ flies further increase their period length, indicating the necessity for continued temperature compensation of PER nuclear export above $25^{\circ} \mathrm{C}$. In contrast, emb-RNAi flies shorten their period length from $25^{\circ} \mathrm{C}$ to $29^{\circ} \mathrm{C}$ by more than one hour. This suggests that at least one other clock protein needs to be exported at temperatures above $25^{\circ} \mathrm{C}$ to avoid speeding up the clock at temperatures above $25^{\circ} \mathrm{C}$. A good candidate would be the unknown CLK-kinase, required to shut down CLK transcriptional activity $(4,39)$. Increased kinase activity with rising temperature would be counteracted by increased nuclear export of the kinase. This idea is also consistent with the phenotype of flies with knocked down emb expression in the per ${ }^{1530 A}$ mutant background: at $29^{\circ} \mathrm{C}$ this resulted in reduction of the per $^{1530 \mathrm{~A}}$-induced period lengthening by $1-2 \mathrm{~h}$, while there was little effect at $25^{\circ} \mathrm{C}$ (Figure $7 \mathrm{C}$, Table 4). Since mutations affecting TIM NES function also show temperature dependent period lengthening from $18^{\circ} \mathrm{C}$ to $29^{\circ} \mathrm{C}$, it is unlikely that blocking TIM nuclear export is responsible for the period shortening observed in emb-RNAi flies above $25^{\circ} \mathrm{C}$. We therefore propose that PER and TIM (presumably as PER:TIM heterodimer and PER:PER homodimer) are both subject to increased nuclear export with increasing temperature.

\section{Molecular consequences of nuclear PER ${ }^{1530 A}$ retention at higher temperatures.}


The temperature-dependent phosphorylation defect of PER ${ }^{1530 A}$ (Figure 3B) combined with its reduced repressor activity (Figure $4 \mathrm{~B}, \mathrm{C}$ ) point to an impairment of this protein to recruit kinases to the CLK transcription factor, which are important for inactivating this transcription factor $(4,39)$. Based on the striking phosphorylation defect of $\mathrm{PER}^{1530 \mathrm{~A}}$ at $25^{\circ} \mathrm{C}$ and $29^{\circ} \mathrm{C}$ (Figure 3B), we propose that the mutant protein is retained in the nucleus and 'protected' from kinases that would normally phosphorylate PER in the cytoplasm to promote nuclear entry (45-48). At $18^{\circ} \mathrm{C}$, where nuclear export is less important, PER ${ }^{1530 A}$ would be normally phosphorylated and imported into to the nucleus. If true, this implies that wild type PER proteins are shuttled in and out of the nucleus at warmer temperatures to prevent early nuclear accumulation and speeding up of the molecular feedback loop with increasing temperature. Overall a relatively simple model emerges in which temperature-dependent enzymatic events (e.g. kinase activities) leading to earlier accumulation of PER and TIM in the nucleus and presumably to premature inactivation of CLK at warm temperatures $\left(>25^{\circ} \mathrm{C}\right)$, are counteracted by increased nuclear export. Although possibly too simplistic, this model is attractive, because it concedes that all enzymes and other factors involved naturally increase their reaction rate and activity with increasing temperature.

\section{Temperature compensation: Network versus cell autonomous property}

The Drosophila circadian clock network driving behavioral activity rhythms consists of several coupled neuronal groups (14). Following Pittendrigh's proposition, it is therefore conceivable that in order to compensate temperature changes some of these groups speed up with temperature, while others slowdown (10). While such a scenario is possible, available evidence points to temperature compensation being a cell autonomous property, affecting 
distinct steps in clock regulation as suggested by others (18). Using the novel LABL technique, we were able to monitor period promoter activity within specific subsets of the clock network and found that all groups are properly temperature compensated. Importantly, this included the DN1 neurons, which on their own are not able to sustain behavioral rhythmicity in DD, as well as peripheral clock cells (Figure 2A, B). Although it can be argued that signals were recorded from specific neuronal subsets, these subsets within the context of the entire network, which could of course influence the oscillations within one group. Nevertheless, even in the background of a $P d f^{01}$ mutation, which largely prevents network coupling due to the lack of the PDF neuropeptide (17), temperature compensated clock gene expression was observed in dorsal subsets of the clock neurons (29). However, the same study revealed that circadian clocks in isolated fly tissues (in halteres and antennae) are over compensated, pointing to differences between neuronal and non-neuronal circadian clocks (29). Taken together, the currently available data support a model of cell autonomous temperature compensation, at least in central circadian clock neurons.

\section{Conclusions:}

The per ${ }^{1530 A}$ NES mutation equally effects temperature compensation on a behavioral and molecular level. Combined with the temperature compensation defects observed after knockdown of the nuclear export factor CRM1, this strongly implicates nuclear export of PER and other clock proteins as important part of the underlying molecular mechanism. Nuclear retention of $\mathrm{PER}^{1530 \mathrm{~A}}$ leads to increased CLK-mediated transcriptional activity, most likely caused by a temperature-dependent PER ${ }^{1530 \mathrm{~A}}$ phosophorylation defect. Our results support a model in which premature nuclear accumulation and CLK-inactivation at warmer 
temperatures is counteracted by increased nuclear export of clock factors, resulting a temperature compensated circadian clock. Future work will reveal, which other clock factors show temperature dependent control of subcellular localization.

\section{Acknowledgements:}

We thank Patrick Emery for discussions and for sharing unpublished results. We thank Isaac Edery and Joanna Chiu for TIM and CLK antibodies, Paul Hardin for Clk ${ }^{\text {out }}$ flies, Paolo SassoneCorsi for the pAc5.1-I530A clone, as well as Mechthild Rosing for technical support. This work was supported by a grant from the Deutsche Forschungsgemeinschaft given to RS (STA 421/71) and a grant from NSERC (RGPIN-2019-06101) to D.T.

\section{Materials and Methods:}

Flies: Pdf-gal4 (49), DvPdf-gal4 (50), R18H11-gal4 (51), tim-UAS-gal4 (30), tim-gal4:27 (52), Clk856-gal4 (53), Clkout (38), per ${ }^{01}$ (19), per ;1-5-1-HA; 2-2-2-myc (43), plo (40), BG-luc (31) , tim-luC (54), and tim-TIM-LUC (36) have been described. UAS-dicer (inserted on the Xchromosome), nos-Cas9, and emb-RNAi lines BL31353 and BL34021 were ordered from the Bloomington Stock Center or from the VDRC (v3347 and v103767). All other fly lines were generated as part of this study.

Generation of per ${ }^{1530}$ and V5-tagged versions of wild per and per ${ }^{1530}$ 
gRNA design and cloning:

Target gRNA sites were selected so that Cas9 mediated cleavage was directed to a target locus of $100 \mathrm{bp}$ upstream and downstream of the per I530A mutation. To avoid off target cleavage optimal target sites were identified using CRISPR target finder (http://flycrispr.molbio.wisc.edu/tools). 2 gRNA targets were chosen that are close to the target locus. Complementary target sites oligos also contained a 5' guanine for transcription from the U6 promoter and a $3 \mathrm{bp}$ overhang compatible to Bbsl sites. Oligos were annealed using standard primer annealing reactions and cloned into Bbsl linearized pCFD3 plasmid (55) via T4 DNA ligation.

Donor plasmid construction:

Donor plasmids that contain the desired per mutations and all elements necessary for homologous recombination were constructed in three subsequent cloning steps. In each round of cloning the $1.5 \mathrm{~kb} 5$ ' homology arm and the $1.5 \mathrm{~kb} 3^{\prime}$ homology arm were individually PCR amplified from $y w$ flies using outside primers HAXbalF and HAHindIIIR in combination with respective internal primers. Outside primers HAXbalF and HAHindIIIR contain a $15 \mathrm{bp}$ overhang for In-fusion cloning that is homolog to linearized vector ends. Inside primers have a 5' $15-20$ bp extensions that are complementary to each other in addition to one defined mutation for each round of cloning. In the initial round of cloning Pam site mutations were introduced to avoid unwanted Cas 9 cleavage within the donor plasmid. The two fragments $\left(5^{\prime}\right.$ homology arm and 3' homology arm) were assembled into plasmid pBS-KS-attB1-2-PT-SA-SD0-2xTY1-V5 (Addgene) that was linearized with Xbal and HindllI using In Fusion cloning. In a second round of cloning the homology arms were amplified again using the pBS donor plasmid 
from the previous round as a template. Outside primers were as described above while the inside primers introduced either a silent HindIII site that can be used to screen for transformants or the V5 tag. In fusion cloning was used to assemble the fragments as described above. The resulting plasmid was then used in a final round of PCR to introduce the per I530A mutation. See table_for a detailed list of all primers.

\section{Embryo injection and screening for transformants:}

Donor plasmids containing the desired mutation along with gRNA plasmids were verified by sequence analysis and scaled up for injections using Qiagen plasmid midiprep. $6 \mu \mathrm{g}$ of each plasmid were precipitated and eluted in injection buffer. gRNA construct and donor plasmids were mixed prior to injection and the mix was injected into freshly laid embryos of nos-Cas9 flies that were crossed to $y w$ flies (55). Surviving adults were backcrossed in batch crosses to FM7; +; + flies to balance $X$-chromosome modifications with FM7. Individual male and female flies from this cross were crossed again to FM7; +; + flies. After letting the females lay eggs for 3-5 days adult transformant flies were used for molecular screening. Two different strategies were used to screen for transformants. In general, a total of 95 flies for each mutation were screened using either PCR or PCR in combination with restriction digests. For per constructs containing the V5 tag a simple PCR approach was used: a 260 bp target locus containing the desired mutations was amplified by PCR using genomic DNA from individual flies. Resulting products were analyzed on agarose gels. PCR products of samples that showed an increase of size due to the $\mathrm{V} 5$ tag being present were then sequenced to verify the presence of the desired mutations and the V5 tag. For per constructs without the V5 tag a 800 bp target locus was amplified by PCR using genomic DNA from individual flies. 1ul of HindIII was then added to 
half of the PCR reaction and incubated for $2 \mathrm{~h}$ at $37^{\circ} \mathrm{C}$. Resulting products were analyzed on agarose gels. The remaining PCR product of samples that showed digested products of the correct size were then sequenced to verify the presence of the desired mutations.

\section{Generation of per ${ }^{1530 A}$ versions tagged with c-Myc and HA epitopes}

In order to perform the PER:PER homodimer assays it was necessary to generate transgenic flies expressing differentially tagged version of per ${ }^{1530 A}$. Constructs encoding wild-type C-Myc and HA tagged version of PER have been described previously (43) and contain the full length tagged per cDNA, expressed under the control 1.3 kb 5'-upstream and 2.1. kb 3' downstream genomic regulatory sequences. To generate constructs encoding C-Myc and HA tagged $\mathrm{PER}^{1530 \mathrm{~A}}$, a $1.1 \mathrm{~kb}$ SanDI/BamHI fragment of the two wild type constructs was replaced with the same fragment containing the I530A mutation (gift from Paolo Sassone-Corsi) exactly as described in (43) and the mutation in the final construct was verified by sequencing. Transgenic flies were generated in a $y w$ background using classical transposase mediated germline transformation (43). Transgenic flies containing per ${ }^{1530 A-m y c}$ on chromosome 2 and per $^{1530 A-H A}$ on chromosome 3 were combined and introduced into per ${ }^{01}$ genetic background using standard genetic crosses.

Table: Primer list

\begin{tabular}{|l|l|l|}
\hline Primer name & Sequence & Used for \\
\hline HAXbalF & Gatggtcgactctagagcggcactgggtgcgta & Outside \\
& & $\begin{array}{l}\text { primer for } \\
\text { amplifying 5' } \\
\text { homology } \\
\text { region }\end{array}$ \\
\hline
\end{tabular}




\begin{tabular}{|c|c|c|}
\hline HAHindIIIR & gatggtcgacaagcttaagcgaaggatgcgggtacA & $\begin{array}{l}\text { Outside } \\
\text { primer for } \\
\text { amplifying } \quad 3^{\prime} \\
\text { homology } \\
\text { region }\end{array}$ \\
\hline perPAMmutF & GGAGGATATCGTGAAGCGTCTGGCGGAGACGGTGT & $\begin{array}{l}\text { Introduces } \\
\text { Pam site } \\
\text { mutation for } \\
\text { gRNA target } 1\end{array}$ \\
\hline perPAMmutR & ACACCGTCTCCGCCAGACGCTTCACGATATCCTCC & $\begin{array}{l}\text { Introduces } \\
\text { Pam site } \\
\text { mutation for } \\
\text { gRNA target } 1\end{array}$ \\
\hline perHindIIIF & CATGTAAGCTTAAGATATCGGAGGAGGCACAGAGC & $\begin{array}{l}\text { Introduces } \\
\text { Hindlll site }\end{array}$ \\
\hline perHindIIIR & ATCTTAAGCTTACATGTGGGCGCCGCCTCGAAGA & $\begin{array}{l}\text { Introduces } \\
\text { Hindlll site }\end{array}$ \\
\hline Per I530AF & Cgcgaatcaaggaggatgccgtgaagcgtctggc & $\begin{array}{l}\text { Introduces } \\
\text { I530A } \\
\text { mutation }\end{array}$ \\
\hline Perl530R & gccagacgcttcacggcatcctccttgattcgcG & $\begin{array}{l}\text { Introduces } \\
\text { I530A } \\
\text { mutation }\end{array}$ \\
\hline perV5F & ACCCCCTGCTGGGCCTGGACTCCACCTGTAAGCTGAAGATATCGGAGGAG & $\begin{array}{l}\text { Introduces V5 } \\
\text { tag into Per }\end{array}$ \\
\hline perV5R & $\begin{array}{l}\text { TCCAGGCCCAGCAGGGGGTTGGGGATGGGCTTGCCTGTGGGCGCCGCCTCG } \\
\text { A }\end{array}$ & $\begin{array}{l}\text { Introduces V5 } \\
\text { tag into Per }\end{array}$ \\
\hline SeqperF1 & GCGTGCCCAAGGACGCCAAGAGCACCTTCT & $\begin{array}{l}\text { Sequences } \\
\text { modified Per } \\
\text { region }\end{array}$ \\
\hline SeqperR1 & ACGGGCGGTGTGATGCCCACCGAGAACGGT & $\begin{array}{l}\text { Sequences } \\
\text { modified Per } \\
\text { region }\end{array}$ \\
\hline $\mathrm{V} 5 \mathrm{~F}$ & GACCCAAGCAGTGCAACGTCTTCGAG & $\begin{array}{l}\text { Molecular } \\
\text { screen of } \\
\text { transformant } \\
\text { s via V5 tag }\end{array}$ \\
\hline V5R & CCGGGACACCTCGTCCATGAGCGTT & $\begin{array}{l}\text { Molecular } \\
\text { screen of } \\
\text { transformant } \\
\text { s via V5 tag }\end{array}$ \\
\hline perscreenHindF1 & AGGACCTCTCAGTCATGAAGGAGACCTACG & $\begin{array}{l}\text { Molecular } \\
\text { screen of } \\
\text { transformant } \\
\text { s via HindIII } \\
\text { site }\end{array}$ \\
\hline $\begin{array}{l}\text { perscreenHindR } \\
1\end{array}$ & TGTACCAGTGCCCGTGCCACCAGTGCCGGC & $\begin{array}{l}\text { Molecular } \\
\text { screen of } \\
\text { transformant } \\
\text { s via HindlII } \\
\text { site }\end{array}$ \\
\hline
\end{tabular}




\begin{tabular}{|l|l|l|}
\hline ScreenTimR2 & GCTGCTTACCGAGCTGAGCGAGTTGCG & $\begin{array}{l}\text { Molecular } \\
\text { screen of } \\
\text { transformant } \\
\text { s }\end{array}$ \\
\hline pergRNAT1F & GTCGACGGGACACCGTCTCCGCC & gRNAtarget \\
\hline pergRNAT1R & AAACGGCGGAGACGGTGTCCCGTC & gRNAtarget \\
\hline
\end{tabular}

\section{Behavior:}

Two to four days old males were loaded into glass tubes containing $5 \%$ sucrose in $2 \%$ agar and loaded into the DAM2 TriKinetics system (Waltham, MA). Flies were exposed to LD for 3 days, followed by 5-7 days in DD to assess their free running periods at constant temperatures of $18^{\circ} \mathrm{C}, 25^{\circ} \mathrm{C}$ or $29^{\circ} \mathrm{C}$. Period length and their significance (RS values) were determined using autocorrelation and Chi-square periodogram analysis functions of the fly tool box implemented in MATLAB (MathWorks). Period values with associated RS values $\geq 1.5$ were considered rhythmic (56). For statistical analysis shown in Figures $1 \mathrm{C}$ and $7 \mathrm{~B}$, estimation statistics has been used. This approach gives a more informative way to analyze and interpret results (57). It focuses on the effect size, as opposed to significance testing. While significance testing ( $p$-values) focus on the acceptance or rejection of the null hypothesis, estimation stats focus on the magnitude of the effect size (i.e. mean difference) and its precision (57). Data were analyzed using DABEST (57), using the website available under https://www.estimationstats.com/\#/ as described (29).

\section{LABL analysis and classical bioluminescence assays:}

LABL: Detailed methods are available in Johnstone et al., 2021 of this issue. Expression of Flipase using the indicated ga/4 drivers allows anatomical restriction of the firefly luciferase reporter (Luc2, Promega). Luminescence of flies were measured using the LumiCycle 32 Color 
(Actimetrics). D-luciferin potassium salt (Gold Biotechnology) was mixed with standard fly food to a final concentration of $15 \mathrm{mM}$ in Drosophila culture plates (Actimetrics). Analysis software was used to normalize the exponential decay, data were exported into .csv files (Actimetrics) and locally written python code was used to organize luminescence data into 30minute bins (LABLv9.py; www.top-lab.org/downloads), and to quantify periods of oscillations using a Morlet wavelet fit (waveletsv4.py; www.top-lab.org/downloads). Data were plotted using Graphpad Prism 9.

Classic Bioluminescence assays: Luciferase expression of individual flies carrying various period-luciferase and timeless-luciferase reporter genes was measured as described previously (41). 3-4 day old males were loaded in 96-well microtiter plates containing $100 \mu \mathrm{l}$ of $5 \%$ sucrose, $1 \%$ agar and $15 \mathrm{mM}$ luciferin. Bioluminescence was detected with a TopCount Multiplate Reader (Perkin Elmer) for serval days during LD and DD conditions at the designated constant temperature. Bioluminescence was measured twice per hour and data were plotted using BRASS ((Version 2.1.3) (58)).

\section{Western Blot}

V5-tagged flies were kept in LD cycles for 3 days at constant temperatures of $18^{\circ} \mathrm{C}, 25^{\circ} \mathrm{C}$ or $29^{\circ} \mathrm{C}$. On the fourth day, flies were collected at the respective time points and frozen in liquid nitrogen. For DD experiments flies were kept in constant darkness for an additional day before collecting sample collection at the respective times. Protein extractions of adult fly heads were performed as previously described (43). For the V5 and TIM blots $\sim 30$ fly heads were homogenized with $50 \mu$ l of Extraction buffer (20 mM HEPES [pH 7.5], $100 \mathrm{mM} \mathrm{KCl,} 5 \%$ glycerin, $10 \mathrm{mM}$ EDTA, $0.1 \%$ Triton-X 100, 5mM DTT, 1x Complete Protease Inhibitor and 4mM Pefabloc. For anti-Clk blots RBS buffer (20 mM HEPES [pH 7.5], $50 \mathrm{mM} \mathrm{KCl,} 10 \%$ glycerin, $2 \mathrm{mM}$ 
EDTA, 1\% Triton-X 100, 0,4\% NP40, 1mM DTT, 1x Complete Protease Inhibitor and $4 \mathrm{mM}$ Pefabloc) was used for extraction. After homogenization samples were sonicated in 3-5 pulses. Protein concentration was measured using Coomassie Plus Protein Assay Reagent (Thermo scientific) and equal amounts of protein were loaded onto $6 \%$ SDS gels for anti-V5 and antiTIM blots or $8 \%$ SDS gels for anti-CLK blots respectively. For anti-V 5 and anti-TIM blots proteins were transferred onto PVDF membranes while for anti-CLK blots proteins were transferred onto Nitrocellulose membranes using a Semi-dry electro blotting unit at $240 \mathrm{~mA}$ for $40 \mathrm{~min}$. After blotting, membranes were blocked with 5\% milk in TBS-T at room temperature for $1 \mathrm{~h}$. First antibodies ( $5 \%$ nonfat milk/TBS-T) were incubated at $4^{\circ} \mathrm{C}$ overnight, followed by secondary antibody ( $5 \%$ nonfat milk/TBS-T) incubation at room temperature for $1,5 \mathrm{~h}$. Dilution of first antibodies were 1:5000 for mouse anti-V5 (Thermo Fisher), 1:2000 for guinea pig antiTIM (59), 1:2000 for mouse anti HA-tag (6E2 Cell signaling), and 1:2000 for anti-CLK (3). The dilutions of HRP-conjugated secondary antibodies were 1:2000 for goat-anti-guinea pig IgG and 1:2000 for anti-mouse IgG (Cell Signaling). Signal Fire ECL substrates (Cell Signaling) were used to develop the membranes. To calculate relative expression levels the individual bands were quantified using Image J. The background was subtracted for each individual lane and the amount of protein was expressed as relative signal intensity by dividing each value by the highest value for each blot (highest value $=100 \%$ ) within one blot. Average values were then calculated from the three individual blots and were blotted as line graphs ( \pm SEM).

\section{Immunoprecipitations}

ColPs were performed as previously described (43). Briefly, adult flies from V5-tagged strains (per ${ }^{V 5}$ and per/530A-V5) were entrained to a 12-h:12-h LD cycle for 3 days at constant temperatures of $18^{\circ} \mathrm{C}, 25^{\circ} \mathrm{C}$ or $29^{\circ} \mathrm{C}$. At ZT 16 or ZT20 $6 \mathrm{ml}$ of flies were collected and frozen in 
liquid nitrogen. Fly heads were separated by repeated vortexing/cooling in liquid nitrogen and $400 \mu \mathrm{l}$ of fly heads were then separated using a $0.45 \mathrm{~mm} / 0.14 \mathrm{~mm}$ metal mesh. Heads were then homogenized in $400 \mu$ l of Extraction Buffer (20 mM Hepes [pH 7.5], $100 \mathrm{mM} \mathrm{KCl}, 1 \mathrm{mM}$ DTT, 5\% glycerol, 0.05\% NP40, 1x Complete Protease Inhibitor and 4 mM Pefabloc.

For the input sample, $20 \mu \mathrm{l}$ are aliquoted and added with $20 \mu \mathrm{l}$ of $2 x$ SDS-sample buffer. The remaining head extract was incubated with Anti-V5 Agarose Affinity Gel (Sigma) ON at $4^{\circ} \mathrm{C}$. Beads were spun down by centrifugation, $20 \mu$ of supernatant was aliquoted and served as the supernatant (unbound) control. The beads containing the bound fraction were washed three times with Extraction Buffer and subsequently resuspended in buffer for further analysis. Co-IPs done to ckeck for homodimers were done the same way except that agarose beads (Protein G Sepharose ${ }^{\mathrm{TM}} 4$ Fast Flow by GE Healthcare) were used that were incubated with mouse anti-myc antibody (9B11, Cell Signaling) for $1 \mathrm{~h}$ at $4^{\circ} \mathrm{C}$ prior to incubating it with the head extracts. The coated beads were then washed with $1 \mathrm{ml}$ of Extraction Buffer, resuspended in $40 \mu \mathrm{L}$ Extraction Buffer/sample and loaded with homogenized head extracts. Individual bands were quantified using Image J and the background was subtracted for each individual lane. Bound protein was normalized to the input and presented as relative signal intensity by dividing each value by the highest value for each blot (highest value $=100 \%$ ). Average values were then calculated from three individual blots and were blotted as bar graphs ( \pm SEM).

\section{Immunostainings:}

The protocol used was the same as previously described (60). Flies were entrained in LD for 6 days. Flies were collected in the dark and stayed in the dark until dissections. Brains were dissected in PBST 0.1\% and fixed for 20 min at room temperature in PFA 4\%. After 3 washes 
brains were blocked for one hour at room temperature in PBST $0.1 \%+5 \%$ goat serum. Primary antibodies were incubated for $48 \mathrm{~h}$ (in PBST $0.1 \%+5 \%$ goat serum) at $4^{\circ} \mathrm{C}$, while secondary incubation was done overnight at $4^{\circ} \mathrm{C}$. Brains were mounted using Vectashield. Monoclonal anti-PDF (DSHB) was used at 1/1000, and pre-absorbed Rabbit anti-PER (61) was used at 1/15000. Secondary antibodies used: goat cross absorbed anti-mice $488++1 / 2000$ (Invitrogen), goat anti-rabbit 555 1/2000 (Invitrogen). Brains were imaged with a Leica TCS SP8 confocal microscope with a $63 x$ objective. Average intensity was measured using ImageJ and quantification was normalized to the background: (signal-background)/background 50 .

\section{Figure Legends:}

Figure 1. Effect of per ${ }^{1530 A}$ on temperature compensation: (A) Cartoon of the PER structure (amino acids 1-1,224) showing the different functional domains including the putative NES and the position of the inserted V5 tag. A magnification of the NES region between residues 510-525 is shown below including a sequence alignment of the putative NES domain from DMPER and mPER1-3. Grey colored regions mark the conserved hydrophobic sites. The position of the Isoleucine 530 (mutated to Alanine in per ${ }^{1530 A}$ ) is shown in red. The alpha-helix formed by the NES is depicted above the sequence alignment. (B) Effect of per ${ }^{1530 A}$ on freerunning locomotor activity analyzed at three different temperatures. Flies were kept for 3-4 days in LD followed by an additional week in DD at the constant temperatures indicated on the left side of each panel. For controls $\left(y w\right.$ and $p e r^{V 5}$ ) and for per $1530 A$ flies (with or without V5 tag) individual actograms of the LD and DD part of the experiments are shown. (C) Period estimates and statistical analysis of individual flies. To analyze the distribution of period values at the different temperatures estimation statistics (ES) was applied. The top part shows the 
individual period data points obtained with the standard error plotted as a line to the right, with the gap indicating the average. The lower part summarizes the median difference between per $^{1530 A}$ and control. The colored curves represent the median difference distribution of the values, and the black vertical line indicates the $95 \%$ confidence interval (see Materials and Methods for details).

Figure 2. per ${ }^{1530 A}$ affects temperature compensation in different neuronal clocks: (A) of Bioluminescence oscillations measured from LABL flies activated by the indicated circadian neuronal drivers (Pdf-Gal4, DvPdf-Gal4 and R18H11-Gal4), in a wild type (per ${ }^{+}$) or mutant $\left(\right.$ per $\left.^{1530 A}\right)$ genetic background, plotted over time. Colors represent mutant flies monitored at different temperatures: $18{ }^{\circ} \mathrm{C}$ (blue), $25^{\circ} \mathrm{C}$ (yellow) or $29^{\circ} \mathrm{C}$ (red). Line thickness represents the mean of two experiments, +/- SEM. (B) Bioluminescence oscillations measured from LABL flies activated by the universal circadian driver tim-UAS-Gal4. Figure details are as described in panel A. (C) Average periods of per-luc oscillations in different neurons. Indicated Gal4 drivers were used to activate LABL in wild type $\left(\right.$ per $\left.^{+}\right)$or mutant $\left(p e r^{1530 A}\right)$ flies. Colors represent different temperatures as described in panel A. Bar represents the mean, +/- SD. Mean values of period $(\tau)$ are denoted underneath the graph.

Figure 3. per $^{1530 \mathrm{~A}}$ causes temperature-dependent defects in oscillation and posttranslational modification of PER. (A) Expression of wild type PER carrying the V5 tag was analyzed at different temperatures. Flies were kept for 3 days in $L D$ at three constant temperatures $\left(18^{\circ} \mathrm{C}\right.$, $25^{\circ} \mathrm{C}$ and $29^{\circ} \mathrm{C}$ ). Protein extracts from adult fly heads were prepared at the indicated Zeitgeber Times (ZT) as described in Materials and Methods. Left panel: western blots comparing PER and TIM temporal expression profiles at different temperatures. Blots were incubated with 
anti-V5 or anti-TIM antibodies, respectively. Right panel: Relative expression levels were plotted as line graphs showing the PER or TIM protein oscillations during a $24 \mathrm{~h}$ day at the three different temperatures. Error bars indicates SEM. (B) PER expression in $\operatorname{per}^{V 5}$ and per $1530 \mathrm{~A}-\mathrm{V} 5$ was compared at the indicated time points and temperatures. Left panels: Protein extract from wild type mutant flies were loaded next to each other for each time point to allow direct comparison. Right panels: Relative expression levels were plotted as line graphs for per $^{V 5}$ and $p e r^{1530-V 5}$ flies. Error bars indicate SEM.

Figure 4: per $^{1530 A}$ causes temperature-dependent dampening of PER-LUC oscillations and impairs repressor function. (A) Bioluminescence rhythms of per $^{+}$and $p e r^{1530 A}$ flies expressing the BG-Iuc fusion protein, reporting temporal PER expression. Individual flies were measured at the indicated temperatures for 2 or 3 days in $12 \mathrm{hr}: 12 \mathrm{hr}$ LD, followed by 4 or 5 days in DD. White bars indicate lights on, black bars lights off, and grey bars subjective day. $\mathrm{N}$ numbers are indicated in parenthesis. (B) Bioluminescence rhythms of $\mathrm{per}^{+}, \mathrm{per}^{01}$, and $p e r^{1530 \mathrm{~A}}$ flies expressing the plo transgene, reporting temporal per transcription and levels. Experiments were performed as in (A). (C) Quantification of plo (left panel) and tim-luc (reporting tim transcription, right panel) average bioluminescence levels for the duration of the entire experiments shown in (B) (plo) and Figure S4C (tim-luc) for the indicated genotypes and temperatures.

Figure 5. per ${ }^{1530 \mathrm{~A}}$ does not disrupt PER:TIM and PER:PER dimerization but effects posttranslational modification of CLK. (A) PER-TIM interactions in perv5 and per $1530 \mathrm{~A}-\mathrm{V5}$ fly heads at the indicated temperatures. Upper panel: $400 \mu \mathrm{l}$ of fly heads were collected at ZT16 
and ZT22 from flies entrained in LD cycles for 3 days and protein extraction was performed as described in Materials and Methods. Protein extracts were loaded onto beads containing antiV5 antibody. Western blot analysis with anti-TIM antibodies to check TIM binding to PER ${ }^{\mathrm{V} 5}$ or PER ${ }^{150 A}$, respectively. Input: total protein extract containing PER and TIM. Unbound: supernatant after binding the extracts to anti-V5 coated beads. Bound: proteins eluted from anti-V5 coated beads. Lower panel: bar graphs representing the relative binding levels calculated from three individual experiments. Values are normalized to total amount of protein loaded in the IPs and are expressed as relative signal intensity (highest value $=100 \%$ ). (B) Homodimerisation of PER and PER ${ }^{1530 A}$ in fly heads at the indicated temperatures. Upper panel: Flies expressing wild type PER (per 01 1-5-1-HA/+;2-2-2-myc/+, lanes 1-3) or PER ${ }^{1530 A}$ (per 01 ;8-6-myc/8-6-myc; 7-3-HA/7-3-HA, lanes 4-6) encoding per transgenes fused to HA and c-myc tags were collected at ZT22 and ColPs experiments were performed essentially as described above. Beads were incubated with anti-c-MYC antibodies and western blots were developed with anti-HA antibodies. 'Input', 'Unbound', and 'Bound' are as described above. Lower panel: bar graphs representing the relative binding levels calculated from three individual experiments. (C) Western blots comparing CLK expression in heads of per ${ }^{V 5}$ and $p^{1530 A-V 5}$ at the indicated temperatures. Flies were entrained in LD cycles for three days and collected at the indicated time points. Extracts from the loss-of-function mutant $\mathrm{Clk}^{\text {out }}$ served as a negative control.

Figure 6: per ${ }^{1530 A}$ modifies subcellular localization of PER in the s-LNv pacemaker neurons in a temperature dependent manner. (A-B) Representative images of PER immunostaining in the s-LNv of control flies (A) and per ${ }^{1530 A}$ flies (B) at two time points, ZT16 and ZT22, and 3 temperatures in LD. Cytoplasmic PDF signals were used as a marker to delimitate the nucleus 
area from the cytoplasmic region. Scale bar: $10 \mu \mathrm{m}$. (C) Box plots of the fraction of nuclear PER over cytoplasmic PER in individual s-LNv cells in controls (left panel) and per ${ }^{1530 A}$ flies (right panel). Each temperature and time point have been done at least twice. Number of cells for $y$ w: $55 \leq \mathrm{N} \leq 98$; Number of cells for per ${ }^{1530 A}: 40 \leq \mathrm{N} \leq 81$.

Figure 7: The nuclear export factor CRM1/EMBARGOED affects temperature compensation.

(A) Effect of downregulating embargoed $(e m b)$ in clock neurons on free-running locomotor activity analyzed at different temperatures. UAS-dicer Clk856-gal4 was used to downregulate emb using two different RNAi lines (BL34021 and v 3347). Flies were kept for 3-4 days in LD followed by an additional week in DD at the constant temperatures indicated on the left side of each panel. Individual actograms of the LD and DD part of the experiments are shown at the three temperatures. (B) Quantification of period length and differences between control (UAS-dicer; Clk856-gal4/+) and emb knockdown flies (UAS-dicer; Clk856-gal4/+; v3347/+) and UAS-dicer; Clk856-gal4/BL34021) using ES (see legend to Figure 1C and Materials and Methods for details). For additional gal4 driver and emb-RNAi lines see Table 3. (C) Free running period length of flies with emb downregulation in the background of the per ${ }^{1530 A}$ mutation. Behavior was analyzed as in (A) and average periods compared between controls (perl530A; Pdf-gal4/+ or per ${ }^{1530 A}$; tim-gal4/+) and per ${ }^{1530 A}$ emb-RNAi knockdown flies (per ${ }^{1530 A} ;$ Pdf-gal4/+; BL31353/+ or per ${ }^{1530 A}$; tim-gal4/+; BL31353/+) at the indicated temperatures. Error bars indicate SEM. For additional emb-RNAi lines see Table 4.

Figure S1. Period of clock oscillations over time, and average clock periods of per': (A) The architecture of the LABL reporter construct. Details of the construction of this reporter are 
reported in Johnstone et al., 2021 of this issue. The period promoter is fused to mCherry with three stop codons (asterisks) and subsequently to Luciferase. The mCherry gene is flanked by FRT recombination sites (triangles), which permit Gal4-mediated flippase excision of the gene, allowing Luciferase expression by the per promoter. (B) Changes of period of clock oscillations in wild type flies, over time. LABL is activated using the indicated Gal4 driver in wild type (per $\left.{ }^{+}\right)$ and mutant $\left(p e r^{1530 A}\right)$ flies, and changes in period of oscillation calculated by Morlett-waveletfitting over time. Colors represent different temperatures: $18^{\circ} \mathrm{C}$ (blue), $25^{\circ} \mathrm{C}$ (yellow) or 29 ${ }^{\circ} \mathrm{C}$ (red). Two replicates are plotted. (C) Average periods of clock oscillations in different neurons of per mutant flies. Indicated Gal4 drivers were used to activate LABL. Colors represent different temperatures as described in panel A. Bar represents the mean, +/- SD.

Figure S2. per ${ }^{1530 A}$ causes temperature-dependent dampening of TIM oscillations. Expression of TIM in per 15 and per $1530 A-V 5$ flies was compared at the indicated time points and temperatures. Left panel: Membranes that were initially blotted with anti-V5 antibodies (see Figure 3B) were stripped and developed with anti-TIM antibodies to analyze the effect of the PER mutation on TIM expression: Right panel: Relative TIM expression levels were plotted as line graphs for per ${ }^{V 5}$ and per $1530-v_{5}$ mutant flies. Error bars indicate SEM.

Figure S3: Analysis of PER and TIM expression in $\operatorname{per}^{V 5}$ and per ${ }^{1530 A-V 5}$ fly heads at different temperatures in DD. Flies were kept for 3 days in LD and for 2 more days in DD at three constant temperatures $\left(18^{\circ} \mathrm{C}, 25^{\circ} \mathrm{C}\right.$ and $\left.29^{\circ} \mathrm{C}\right)$. Left panels: Protein extracts from per ${ }^{V 5}$ and per $1530 \mathrm{~A}-\mathrm{V} 5$ heads were loaded next to each other for each time point to allow direct comparison. Blots were developed with anti-V5 or anti-TIM antibodies, respectively. Right 
panels: Relative expression levels were blotted as line graphs for per $^{V 5}$ and per $1530 A-v 5$ flies. Error bars indicate SEM.

Figure S4: per ${ }^{1530 A}$ causes temperature-dependent dampening of TIM-LUC oscillations and impairs repressor function. (A) Bioluminescence rhythms of per $^{+}$and $p e r^{1530 A}$ flies expressing the tim-TIM-LUC fusion protein, reporting temporal TIM expression. Individual flies were measured at the indicated temperatures for 4 days in $12 \mathrm{hr}: 12 \mathrm{hr}$ LD, followed by 2 or 3 days in DD. White bars indicate lights on, black bars lights off, and grey bars subjective day. $\mathrm{N}$ numbers are indicated in parenthesis. (B) Bioluminescence rhythms of $p e r^{+}, p^{01}$, and $p e r^{1530 A}$ flies expressing the tim-TIM-LUC fusion protein for 3 days in LD followed by 3 days in DD at $25^{\circ} \mathrm{C}$. Labelling as in (A). (C) Bioluminescence rhythms of $p e r^{+}, p e r^{01}$, and $p e r^{1530 A}$ flies expressing the tim-luc transgene, reporting tim transcription. Flies were recorded for 2 or 3 days in LD followed by 3 days in DD at the indicated temperatures. Labelling as in (A).

Figure S5: Relative PER level in the s-LNv in control flies and per ${ }^{1530 A}$ flies. Same cells as in Figure 6 were quantified. Note that in control flies PER levels appear reduced at ZT22 while the fraction of nuclear PER over cytoplasmic PER is constant at all three temperatures (Figure 6). 
Table 1: Free-running behavioral rhythms of per ${ }^{1530}$ mutants and controls

\begin{tabular}{|l|c|c|c|c|c|}
\hline Genotype & Temp $\left.^{\circ} \mathbf{C}\right)$ & $\mathbf{n}$ & Tau (hr \pm SEM) & RS \pm SEM & \% rhythmic \\
\hline$y w$ & 18 & 30 & $24.1 \pm 0.2$ & $3.0 \pm 0.2$ & 83.3 \\
\hline & 25 & 32 & $23.5 \pm 0.1$ & $2.6 \pm 0.2$ & 71.9 \\
\hline per $^{1530 A}$ & 29 & 29 & $23.7 \pm 0.1$ & $2.8 \pm 0.2$ & 93.1 \\
\hline & 18 & 38 & $23.0 \pm 0.4$ & $2.2 \pm 0.2$ & 71.1 \\
\hline & 25 & 35 & $25.9 \pm 0.3$ & $2.4 \pm 0.3$ & 80.0 \\
\hline per $^{V 5}$ & 29 & 37 & $27.9 \pm 0.3$ & $1.8 \pm 0.1$ & 59.5 \\
\hline & 18 & 44 & $23.0 \pm 0.1$ & $3.4 \pm 0.2$ & 97.7 \\
\hline & 25 & 47 & $23.2 \pm 0.1$ & $3.5 \pm 0.2$ & 93.6 \\
\hline perl530A-V5 & 29 & 43 & $23.3 \pm 0.1$ & $3.4 \pm 0.3$ & 83.7 \\
\hline & 18 & 78 & $23.5 \pm 0.1$ & $3.0 \pm 0.2$ & 80.8 \\
\hline & 25 & 68 & $26.7 \pm 0.2$ & $2.3 \pm 0.2$ & 75.0 \\
\hline & 29 & 47 & $29.4 \pm 0.3$ & $1.9 \pm 0.2$ & 57.4 \\
\hline
\end{tabular}

Table 2: Free-running behavioral rhythms of period mutants with altered phosphorylation sites

\begin{tabular}{|l|c|c|c|c|c|}
\hline Genotype & Temp $\left({ }^{\circ} \mathbf{C}\right)$ & $\mathbf{n}$ & Tau (h \pm SEM) & RS \pm SEM & \% rhythmic \\
\hline per $^{\text {S596A }}$ & 18 & 31 & $17.7 \pm 0.4$ & $4.3 \pm 0.2$ & 90.3 \\
\hline & 25 & 32 & $16.9 \pm 0.3$ & $3.8 \pm 0.2$ & 100 \\
\hline & 29 & 27 & $16.0 \pm 0.1$ & $3.0 \pm 0.2$ & 88.9 \\
\hline per $^{\text {TS583-596 }}$ & 18 & 26 & $17.2 \pm 0.1$ & $4.7 \pm 0.3$ & 88.5 \\
\hline & 25 & 25 & $16.7 \pm 0.1$ & $4.4 \pm 0.2$ & 100 \\
\hline per $^{547 A}$ & 29 & 26 & $16.5 \pm 0.2$ & $3.1 \pm 0.2$ & 73.1 \\
\hline & 18 & 44 & $29.7 \pm 0.2$ & $3.4 \pm 0.2$ & 100 \\
\hline & 25 & 47 & $30.9 \pm 0.1$ & $4.9 \pm 0.2$ & 100 \\
\hline & 29 & 43 & $31.6 \pm 0.2$ & $2.7 \pm 0.3$ & 100 \\
\hline
\end{tabular}


Table 3: Free-running behavioral rhythms of embargoed RNAi lines and controls

\begin{tabular}{|c|c|c|c|c|c|}
\hline Genotype & Temp ( $\left.{ }^{\circ} \mathrm{C}\right)$ & $\mathbf{n}$ & Tau (h \pm SEM) & RS \pm SEM & \% rhythmic \\
\hline \multirow[t]{3}{*}{ UAS-dicer; Clk856-Gal4/+; BL31353/+ } & 18 & 40 & $24.7 \pm 0.8$ & $1.7 \pm 0.2$ & 42.5 \\
\hline & 25 & 39 & $26.3 \pm 0.2$ & $2.3 \pm 0.2$ & 97.4 \\
\hline & 29 & 40 & $24.8 \pm 0.2$ & $2.7 \pm 0.2$ & 97.5 \\
\hline \multirow[t]{3}{*}{ UAS-dicer; tim-Gal4/+; BL31353/+ } & 18 & 41 & $21.8 \pm 0.4$ & $1.9 \pm 0.1$ & 46.3 \\
\hline & 25 & 37 & $25.6 \pm 0.3$ & $2.0 \pm 0.2$ & 75.7 \\
\hline & 29 & 42 & $24.1 \pm 0.1$ & $2.4 \pm 0.2$ & 97.6 \\
\hline \multirow[t]{3}{*}{ UAS-dicer; Pdf-Gal4/+; BL31353/+ } & 18 & 25 & $22.6 \pm 0.9$ & $1.6 \pm 0.1$ & 52.0 \\
\hline & 25 & 27 & $25.1 \pm 0.2$ & $3.1 \pm 0.3$ & 81.5 \\
\hline & 29 & 25 & $23.8 \pm 0.1$ & $3.3 \pm 0.3$ & 96.0 \\
\hline \multirow[t]{5}{*}{ UAS-dicer; Clk856-Gal4/+; v3347/+ } & 18 & 14 & $23.6 \pm 0.4$ & $1.6 \pm 0.2$ & 78.6 \\
\hline & 20 & 23 & $26.3 \pm 0.2$ & $2.4 \pm 0.2$ & 100 \\
\hline & 22 & 49 & $26.9 \pm 0.1$ & $2.7 \pm 0.1$ & 98.0 \\
\hline & 25 & 32 & $26.7 \pm 0.1$ & $3.8 \pm 0.1$ & 100 \\
\hline & 29 & 31 & $25.1 \pm 0.1$ & $3.7 \pm 0.1$ & 100 \\
\hline \multirow[t]{3}{*}{ UAS-dicer; tim-Gal4/v3347;+ } & 18 & 21 & $23.9 \pm 0.3$ & $2.3 \pm 0.3$ & 33.3 \\
\hline & 25 & 20 & $26.3 \pm 0.4$ & $2.6 \pm 0.3$ & 60.0 \\
\hline & 29 & 14 & $23.7 \pm 0.3$ & $2.4 \pm 0.4$ & 92.9 \\
\hline \multirow[t]{3}{*}{ UAS-dicer; Pdf-Gal4/v3347;+ } & 18 & 30 & $24.7 \pm 0.5$ & $2.1 \pm 0.1$ & 60 \\
\hline & 25 & 29 & $25.4 \pm 0.1$ & $4.4 \pm 0.2$ & 100 \\
\hline & 29 & 29 & $24.3 \pm 0.1$ & $3.8 \pm 0.3$ & 96.6 \\
\hline \multirow[t]{5}{*}{ UAS-dicer; Clk856-Gal4/BL34021;+ } & 18 & 32 & $24.2 \pm 0.5$ & $1.7 \pm 0.1$ & 40.6 \\
\hline & 20 & 9 & $26.4 \pm 1.2$ & $1.8 \pm 0.2$ & 69.2 \\
\hline & 22 & 13 & $27.7 \pm 0.4$ & $1.7 \pm 0.2$ & 81.3 \\
\hline & 25 & 59 & $27.3 \pm 0.2$ & $1.9 \pm 0.1$ & 76.3 \\
\hline & 29 & 63 & $24.9 \pm 0.2$ & $2.5 \pm 0.2$ & 82.5 \\
\hline \multirow[t]{3}{*}{ UAS-dicer; tim-Gal4/+; BL34021/+ } & 18 & 22 & $22.8 \pm 0.5$ & $1.9 \pm 0.2$ & 77.3 \\
\hline & 25 & 20 & $26.5 \pm 0.4$ & $2.3 \pm 0.3$ & 70.0 \\
\hline & 29 & 21 & $25.0 \pm 0.3$ & $2.5 \pm 0.4$ & 61.9 \\
\hline \multirow[t]{3}{*}{ UAS-dicer; Pdf-Gal4/+; BL34021/+ } & 18 & 31 & $24.3 \pm 0.5$ & $1.7 \pm 0.1$ & 51.6 \\
\hline & 25 & 29 & $26.5 \pm 0.4$ & $2.1 \pm 0.1$ & 82.8 \\
\hline & 29 & 32 & $24.0 \pm 0.1$ & $3.1 \pm 0.2$ & 90.6 \\
\hline
\end{tabular}




\begin{tabular}{|c|c|c|c|c|c|}
\hline \multirow[t]{3}{*}{ UAS-dicer; Clk856-Gal4/v103767;+ } & 18 & 31 & $22.6 \pm 0.9$ & $1.9 \pm 0.2$ & 29.0 \\
\hline & 25 & 30 & $26.5 \pm 0.4$ & $2.0 \pm 0.2$ & 83.3 \\
\hline & 29 & 29 & $25.9 \pm 0.3$ & $2.1 \pm 0.2$ & 89.7 \\
\hline \multirow[t]{3}{*}{ UAS-dicer; tim-Gal4/v103767;+ } & 18 & 14 & $22.1 \pm 0.5$ & $1.6 \pm 0.1$ & 28.6 \\
\hline & 25 & 13 & $27.2 \pm 0.4$ & $1.6 \pm 0.1$ & 69.2 \\
\hline & 29 & 6 & $24.8 \pm 1.0$ & $2.1 \pm 0.4$ & 33.3 \\
\hline \multirow[t]{3}{*}{ UAS-dicer; Pdf-Gal4/v103767;+ } & 18 & 16 & $23.8 \pm 1.2$ & $1.6 \pm 0.1$ & 56.3 \\
\hline & 25 & 16 & $25.5 \pm 0.3$ & $3.1 \pm 0.3$ & 81.3 \\
\hline & 29 & 14 & $25.0 \pm 0.4$ & $2.2 \pm 0.3$ & 100.0 \\
\hline \multirow[t]{4}{*}{ UAS-dicer; Clk856-Gal4/+ } & 18 & 14 & $24.0 \pm 0.3$ & $2.4 \pm 0.2$ & 100 \\
\hline & 22 & 10 & $24.0 \pm 0.3$ & $3.8 \pm 0.3$ & 100 \\
\hline & 25 & 15 & $23.6 \pm 0.1$ & $3.8 \pm 0.2$ & 100 \\
\hline & 29 & 14 & $23.4 \pm 0.1$ & $3.5 \pm 0.3$ & 100 \\
\hline \multirow[t]{3}{*}{ UAS-dicer; tim-Gal4/+ } & 18 & 13 & $24.1 \pm 0.4$ & $2.1 \pm 0.2$ & 100 \\
\hline & 25 & 13 & $24.3 \pm 0.1$ & $3.3 \pm 0.2$ & 100 \\
\hline & 29 & 13 & $24.0 \pm 0.1$ & $3.5 \pm 0.2$ & 100 \\
\hline \multirow[t]{3}{*}{ UAS-dicer; Pdf-gal4/+ } & 18 & 16 & $22.7 \pm 0.4$ & $2.4 \pm 0.2$ & 87.5 \\
\hline & 25 & 15 & 24. $7 \pm 0.1$ & $2.8 \pm 0.2$ & 100 \\
\hline & 29 & 11 & $24.0 \pm 0.1$ & $3.9 \pm 0.4$ & 90.9 \\
\hline \multirow[t]{3}{*}{$B L 31353 /+$} & 18 & 23 & $23.1 \pm 0.1$ & $2.3 \pm 0.2$ & 82,6 \\
\hline & 25 & 25 & $23.5 \pm 0.1$ & $3.3 \pm 0.2$ & 100 \\
\hline & 29 & 26 & $23.4 \pm 0.1$ & $3.4 \pm 0.1$ & 100 \\
\hline \multirow[t]{3}{*}{ V3347/+ } & 18 & 16 & $23.9 \pm 0.3$ & $2.0 \pm 0.2$ & 100 \\
\hline & 25 & 15 & $24.1 \pm 0.1$ & $3.7 \pm 0.3$ & 100 \\
\hline & 29 & 15 & $23.6 \pm 0.1$ & $3.8 \pm 0.4$ & 100 \\
\hline \multirow[t]{3}{*}{$B L 34021 /+$} & 18 & 32 & $23.7 \pm 0.2$ & $2.2 \pm 0.2$ & 84.4 \\
\hline & 25 & 32 & $23.8 \pm 0.2$ & $2.6 \pm 0.2$ & 93.8 \\
\hline & 29 & 31 & $23.9 \pm 0.3$ & $3.2 \pm 0.2$ & 96.8 \\
\hline
\end{tabular}


Table 4: Free-running behavioral rhythms of per ${ }^{1530 A}$ emb-RNAi double mutants and controls

\begin{tabular}{|c|c|c|c|c|c|}
\hline Genotype & $\operatorname{Temp}\left({ }^{\circ} \mathrm{C}\right)$ & $\mathbf{n}$ & Tau (h \pm SEM) & RS \pm SEM & \% rhythmic \\
\hline \multirow[t]{3}{*}{ per ${ }^{1530 A} ;$ Pdf-gal4/+ } & 18 & 15 & $24.7 \pm 0.1$ & $4.2 \pm 0.3$ & 100 \\
\hline & 25 & 16 & $26.4 \pm 0.1$ & $3.6 \pm 0.3$ & 100 \\
\hline & 29 & 9 & $31.0 \pm 0.7$ & $1.6 \pm 0.3$ & 55.6 \\
\hline \multirow[t]{3}{*}{ peri530A; Pdf-gal4/+, BL31353/+ } & 18 & 14 & $24.1 \pm 0.2$ & $2.5 \pm 0.4$ & 100 \\
\hline & 25 & 15 & $25.1 \pm 0.2$ & $3.0 \pm 0.3$ & 93.3 \\
\hline & 29 & 14 & $27.1 \pm 0.7$ & $1.5 \pm 0.2$ & 42.9 \\
\hline \multirow[t]{3}{*}{ per ${ }^{1530 A} ;$ Pdf-gal4/+, BL34021/+ } & 18 & 12 & $22.8 \pm 0.7$ & $2.5 \pm 0.4$ & 75.0 \\
\hline & 25 & 14 & $\mathrm{n} / \mathrm{a}$ & $\mathrm{n} / \mathrm{a}$ & 0 \\
\hline & 29 & 15 & $24.9 \pm 0.6$ & $1.2 \pm 0.2$ & 53.3 \\
\hline \multirow[t]{3}{*}{ peri530A; tim-gal4/+ } & 18 & 13 & $22.9 \pm 0.3$ & $2.0 \pm 0.4$ & 92.3 \\
\hline & 25 & 16 & $26.7 \pm 0.2$ & $2.4 \pm 0.3$ & 68.8 \\
\hline & 29 & 14 & $29.8 \pm 0.4$ & $1.8 \pm 0.3$ & 64.3 \\
\hline \multirow[t]{3}{*}{ per ${ }^{1530 A}$; tim-gal4/+, BL31353/+ } & 18 & 16 & $24.4 \pm 0.2$ & $3.9 \pm 0.6$ & 87.5 \\
\hline & 25 & 14 & $25.6 \pm 0.2$ & $2.9 \pm 0.3$ & 100 \\
\hline & 29 & 8 & $27.9 \pm 0.3$ & $1.4 \pm 0.5$ & 87.5 \\
\hline
\end{tabular}

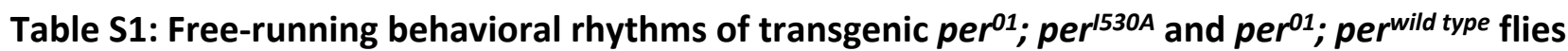

\begin{tabular}{|l|l|l|l|l|l|}
\hline Genotype & Temp $\left({ }^{\circ} \mathbf{C}\right)$ & $\mathbf{n}$ & Tau (h \pm SEM) & RS \pm SEM & \% rhythmic \\
\hline per ${ }^{01} ; 1-5-1 /+; 2-2-2 /+$ (wild type) & 18 & 32 & $22.9 \pm 0.3$ & $1.9 \pm 0.1$ & 87.5 \\
\hline & 25 & 32 & $23.2 \pm 0.1$ & $1.9 \pm 0.1$ & 87.5 \\
\hline per $^{01} ; 8-6 ; 7-3\left(\right.$ per $\left.^{1530 A}\right)$ & 29 & 31 & $22.8 \pm 0.1$ & $2.6 \pm 0.2$ & 83.9 \\
\hline & 18 & 50 & $24.9 \pm 0.4$ & $1.9 \pm 0.1$ & 60.0 \\
\hline & 25 & 55 & $25.7 \pm 0.3$ & $2.5 \pm 0.2$ & 65.5 \\
\hline & 29 & 56 & $27.4 \pm 0.3$ & $1.6 \pm 0.2$ & 35.7 \\
\hline
\end{tabular}




\section{References:}

1. Peschel N, Helfrich-Förster C. Setting the clock - by nature: Circadian rhythm in the fruitfly Drosophila melanogaster. FEBS Letters. 2011;585(10):1435-42.

2. Tataroglu O, Emery P. The molecular ticks of the Drosophila circadian clock. Curr Opin Insect Sci. 2015;7:51-7.

3. Cai YD, Xue Y, Truong CC, Del Carmen-Li J, Ochoa C, Vanselow JT, et al. CK2 Inhibits TIMELESS Nuclear Export and Modulates CLOCK Transcriptional Activity to Regulate Circadian Rhythms. Curr Biol. Februar 2021;31(3):502-514.e7.

4. Yu W, Zheng H, Houl J, Dauwalder B, Hardin P. PER-dependent rhythms in CLK phosphorylation and E-box binding regulate circadian transcription. Genes Dev. 2006;20:723-33.

5. Chiu JC, Ko HW, Edery I. NEMO/NLK Phosphorylates PERIOD to Initiate a Time-Delay Phosphorylation Circuit that Sets Circadian Clock Speed. Cell. 2011;145(3):357-70.

6. Grima B, Lamouroux A, Chelot E, Papin C, B L-B, Rouyer F. The F-box protein slimb controls the levels of clock proteins period and timeless. Nature. 2002;420:178-82.

7. Hall J. Circadian pacemakers blowing hot and cold-but they're clocks, not thermometers. Cell. 1997;90:9-12.

8. Hastings J, Sweeney B. ON THE MECHANISM OF TEMPERATURE INDEPENDENCE IN A BIOLOGICAL CLOCK. Proc National Acad Sci. 1957;43(9):804-11.

9. Pittendrigh CS. ON TEMPERATURE INDEPENDENCE IN THE CLOCK SYSTEM CONTROLLING EMERGENCE TIME IN DROSOPHILA. Proc Natl Acad Sci USA. Oktober 1954;40(10):101829.

10. Zimmerman $\mathrm{W}$, Pittendrigh $\mathrm{C}$, Pavlidis $\mathrm{T}$. Temperature compensation of the circadian oscillation in drosophila pseudoobscura and its entrainment by temperature cycles. $J$ Insect Physiol. 1968;14:669-84.

11. Kurosawa G, Iwasa Y. Temperature compensation in circadian clock models. J Theor Biol. 2005;233(4):453-68.

12. Bünning E. The Physiological Clock [Internet]. Berlin, Heidelberg: Springer Berlin Heidelberg; 1964. (Heidelberg Science Library). http://link.springer.com/10.1007/978-3662-22511-0

13. Top D, Young MW. Coordination between Differentially Regulated Circadian Clocks Generates Rhythmic Behavior. Cold Spring Harb Perspect Biol. 2018;10(7):a033589.

14. Yao Z, Shafer O. The Drosophila Circadian Clock Is a Variably Coupled Network of Multiple Peptidergic Units. Science. 2014;343(6178):1516-20. 
15. Renn S, Park J, Rosbash M, Hall J, Taghert P. A pdf Neuropeptide Gene Mutation and Ablation of PDF Neurons Each Cause Severe Abnormalities of Behavioral Circadian Rhythms in Drosophila. Cell. 1999;99(7):791-802.

16. Veleri S, Brandes C, C H-F, Hall J, Stanewsky R. A self-sustaining, light-entrainable circadian oscillator in theDrosophilabrain. Curr Biol. 2003;13:1758-67.

17. Yoshii T, Wülbeck C, Sehadova H, Veleri S, Bichler D, Stanewsky R, et al. The neuropeptide pigment-dispersing factor adjusts period and phase of Drosophila's clock. J Neurosci. 2009;29(8):2597-610.

18. Kidd $\mathrm{P}$, Young $\mathrm{M}$, Siggia $\mathrm{E}$. Temperature compensation and temperature sensation in the circadian clock. Proc National Acad Sci. 2015;112(46):E6284-92.

19. Konopka R, Benzer S. Clock mutants of Drosophila melanogaster. Proc Natl Acad Sci U S A. $1971 ; 68: 2112-6$.

20. Curtin K, Huang Z, Rosbash M. Temporally regulated nuclear entry of theDrosophilaperiodprotein contributes to the circadian clock. Neuron. 1995;14:365-72.

21. Konopka R, Pittendrigh C, Orr D. Reciprocal behaviour associated with altered homeostasis and photosensitivity ofDrosophilaclock mutants. J Neurogenet. 1989;6:110.

22. Gekakis N, Saez L, AM D-B, Myers M, Sehgal A, Young M, et al. Isolation of timeless by PER protein interaction: defective interaction between TIMELESS protein and longperiod mutant PERL. Science. 1995;270:811-5.

23. Singh S, Giesecke A, Damulewicz M, Fexova S, Mazzotta GM, Stanewsky R, et al. New Drosophila Circadian Clock Mutants Affecting Temperature Compensation Induced by Targeted Mutagenesis of Timeless. Front Physiol. 2019;10:1442.

24. Ashmore L, Sathyanarayanan S, Silvestre D, Emerson M, Schotland P, Sehgal A. Novel insights into the regulation of the timeless protein. J Neurosci. 2003;23:7810-9.

25. Vielhaber E, Duricka D, Ullman K, Virshup D. Nuclear Export of Mammalian PERIOD Proteins. J Biol Chem. 2001;276(49):45921-7.

26. Yagita K, Tamanini F, Yasuda M, Hoeijmakers J, Horst G, Okamura H. Nucleocytoplasmic shuttling and mCRY-dependent inhibition of ubiquitylation of the MPER2 clock protein. Embo J. 2002;21(6):1301-14.

27. Saez L, Young M. Regulation of nuclear entry of the Drosophila clock proteins PERIOD and TIMELESS. Neuron. 1996;17:911-20.

28. Yildiz Ö, Doi M, Yujnovsky I, Cardone L, Berndt A, Hennig S, et al. Crystal Structure and Interactions of the PAS Repeat Region of the Drosophila Clock Protein PERIOD. Mol Cell. 2005;17(1):69-82. 
29. Versteven M, Ernst K-M, Stanewsky R. A Robust and Self-Sustained Peripheral Circadian Oscillator Reveals Differences in Temperature Compensation Properties with Central Brain Clocks. iScience. 21. August 2020;23(8):101388.

30. Blau J, Young M. Cycling vrille Expression Is Required for a Functional Drosophila Clock. Cell. 1999;99(6):661-71.

31. Stanewsky R, Jamison C, Plautz J, Kay S, Hall J. Multiple circadian-regulated elements contribute to cycling period gene expression in Drosophila. Embo J. 1997;16:5006-18.

32. Majercak J, Sidote D, Hardin P, Edery I. How a Circadian Clock Adapts to Seasonal Decreases in Temperature and Day Length. Neuron. 1999;24(1):219-30.

33. Edery I, Zwiebel L, Dembinska M, Rosbash M. Temporal phosphorylation of the Drosophila period protein. Proc Natl Acad Sci U S A. 1994;91:2260-4.

34. Abrieux A, Xue Y, Cai Y, Lewald KM, Nguyen HN, Zhang Y, u. a. EYES ABSENT and TIMELESS integrate photoperiodic and temperature cues to regulate seasonal physiology in Drosophila. Proc Natl Acad Sci USA. 2020;117(26):15293-304.

35. Martin Anduaga A, Evantal N, Patop IL, Bartok O, Weiss R, Kadener S. Thermosensitive alternative splicing senses and mediates temperature adaptation in Drosophila. eLife. 2019;8:e44642.

36. Lamba P, Foley LE, Emery P. Neural Network Interactions Modulate CRY-Dependent Photoresponses in Drosophila. J Neurosci. 04 2018;38(27):6161-71.

37. Zeng H, Qian Z, Myers M, Rosbash M. A light-entrainment mechanism for the Drosophila circadian clock. Nature. 1996;380:129-35.

38. Mahesh G, Jeong E, Ng F, Liu Y, Gunawardhana K, Houl J, u. a. Phosphorylation of the transcription activator CLOCK regulates progression through a approximately 24- $h$ feedback loop to influence the circadian period in Drosophila. J Biol Chem. 2014;289(28):19681-93.

39. Yu W, Zheng $\mathrm{H}$, Price JL, Hardin PE. DOUBLETIME plays a noncatalytic role to mediate CLOCK phosphorylation and repress CLOCK-dependent transcription within the Drosophila circadian clock. Mol Cell Biol. März 2009;29(6):1452-8.

40. Brandes C, Plautz J, Stanewsky R, Jamison C, Straume M, Wood K, u. a. Novel features of Drosophila period transcription revealed by real-time luciferase reporting. Neuron. 1996;16:687-92.

41. Stanewsky R, Lynch K, Brandes C, Hall J. Mapping of elements involved in regulating normal temporalperiodandtimelessRNA expression patterns in Drosophila melanogaster. J Biol Rhythms. 2002;17:293-306.

42. Shafer O, Rosbash M, Truman J. Sequential nuclear accumulation of the clock proteins Period and Timeless in the pacemaker neurons of Drosophila melanogaster. J. Neurosci. 2002;22:5946-54. 
43. Landskron J, Chen K, Wolf E, Stanewsky R. A Role for the PERIOD:PERIOD Homodimer in the Drosophila Circadian Clock. Plos Biol. 2009;7(4):e1000003.

44. Dietzl G, Chen D, Schnorrer F, Su K, Barinova Y, Fellner $M$, et al. A genome-wide transgenic RNAi library for conditional gene inactivation in Drosophila. Nature. 2007;448(7150):151-6.

45. Akten B, Jauch E, Genova G, Kim E, Edery I, Raabe T, et al. A role for CK2 in the Drosophila circadian oscillator. Nat Neurosci. 2003;6(3):251-7.

46. Ko H, Kim E, Chiu J, Vanselow J, Kramer A, Edery I. A Hierarchical Phosphorylation Cascade That Regulates the Timing of PERIOD Nuclear Entry Reveals Novel Roles for ProlineDirected Kinases and GSK-3ß/SGG in Circadian Clocks. J Neurosci. 2010;30(38):1266475.

47. Lin J, Kilman V, Keegan K, Paddock B, M E-L, Rosbash M, et al. A role for casein kinase 2a in the Drosophila circadian clock. Nature. 2002;420:816-20.

48. Lin J, Schroeder A, Allada R. In vivo circadian function of casein kinase 2 phosphorylation sites in Drosophila PERIOD. J. Neurosci. 2005;25:11175-83.

49. Park J, C H-F, Lee G, Liu L, Rosbash M, Hall J. Differential regulation of circadian pacemaker output by separate clock genes in Drosophila. Proc Natl Acad Sci U S A. 2000;97:3608-13.

50. Guo F, Cerullo I, Chen X, Rosbash M. PDF neuron firing phase-shifts key circadian activity neurons in Drosophila. Elife. 2014;3(0):e02780.

51. Guo F, Yu J, Jung H, Abruzzi K, Luo W, Griffith L, et al. Circadian neuron feedback controls the Drosophila sleep-activity profile. Nature. 2016;536(7616):292-7.

52. Kaneko M, Hall J. Neuroanatomy of cells expressing clock genes in Drosophila: transgenic manipulation of the period and timeless genes to mark the perikarya of circadian pacemaker neurons and their projections. J Comp Neurol. 2000;422:66-94.

53. Gummadova J, Coutts G, Glossop N. Analysis of the Drosophila Clock Promoter Reveals Heterogeneity in Expression between Subgroups of Central Oscillator Cells and Identifies a Novel Enhancer Region. J Biol Rhythms. 2009;24(5):353-67.

54. Stanewsky R, Kaneko M, Emery P, Beretta B, K W-S, Kay S, et al. The cryb mutation identifies cryptochrome as a circadian photoreceptor in Drosophila. Cell. 1998;95:68192.

55. Port F, Chen H, Lee T, Bullock S. Optimized CRISPR/Cas tools for efficient germline and somatic genome engineering in Drosophila. Proc Natl Acad Sci U S A. 2014;111(29):E2967-76.

56. Levine J, Funes P, Dowse H, Hall J. Signal analysis of behavioral and molecular cycles. BMC Neurosci. 2002;3:1. 
57. Ho J, Tumkaya T, Aryal S, Choi H, Claridge-Chang A. Moving beyond P values: data analysis with estimation graphics. Nat Methods. Juli 2019;16(7):565-6.

58. Locke J, Southern M, L K-B, Hibberd V, Brown P, Turner M, et al. Extension of a genetic network model by iterative experimentation and mathematical analysis. Mol Syst Biol. 2005;1:2005 0013.

59. Sidote D, Majercak J, Parikh V, Edery I. Differential effects of light and heat on the Drosophila circadian clock proteins PER and TIM. Mol Cell Biol. 1998;18:2004-13.

60. Lamaze A, Öztürk-Çolak A, Fischer R, Peschel N, Koh K, Jepson JEC. Regulation of sleep plasticity by a thermo-sensitive circuit in Drosophila. Sci Rep. Februar 2017;7(1):40304.

61. Stanewsky R, Frisch B, Brandes C, MJ H-C, Rosbash M, Hall J. Temporal and spatial expression patterns of transgenes containing increasing amounts of the Drosophila clock gene period and a lacZ reporter: mapping elements of the PER protein involved in circadian cycling. J. Neurosci. 1997;17:676-96. 


\section{Figure 1}

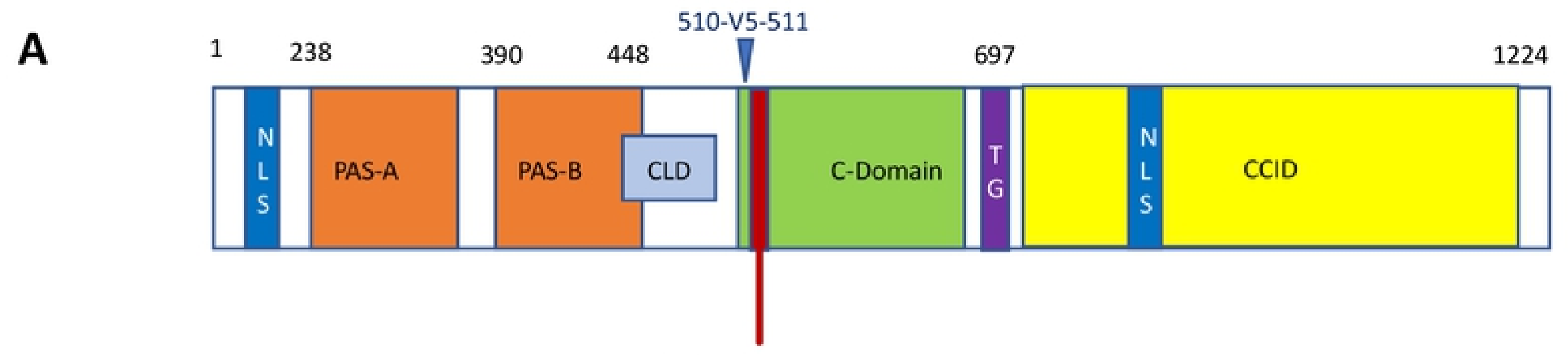

Putative NES : 524-534

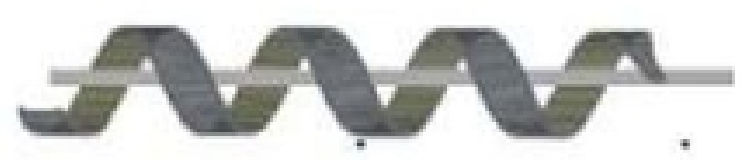

1530

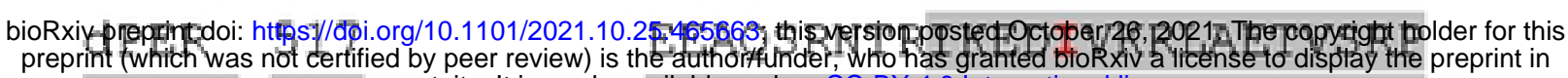

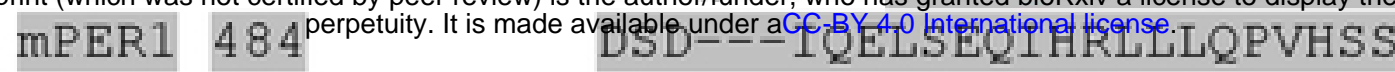

MPER2 454 HPS---VQELTEQIHRLLMQPVPHS

MPER3 394 KD----IAELQEQIHKLLLQPVHAS

NES

B

\section{yw}

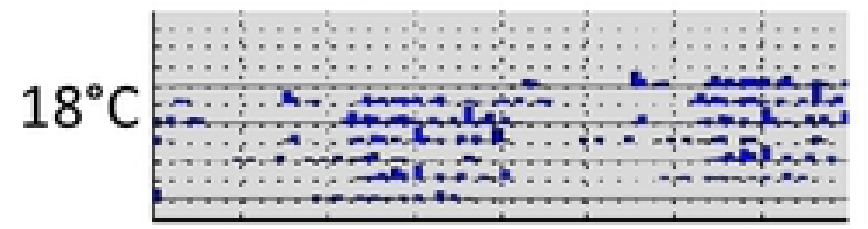

$25^{\circ} \mathrm{C}$

$29^{\circ} \mathrm{C}$

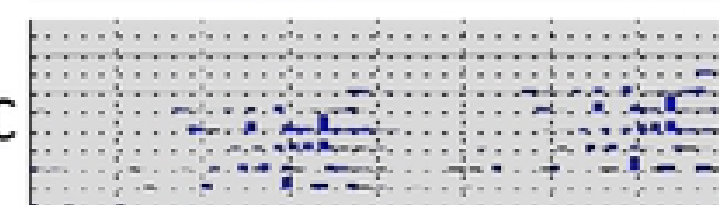

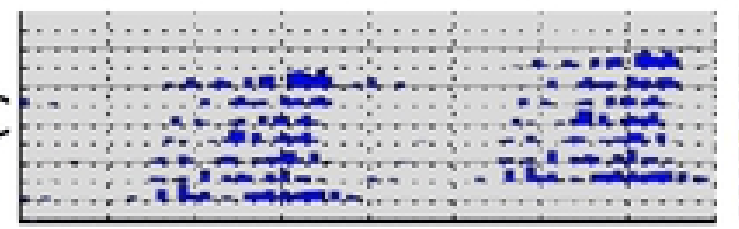

$\operatorname{per}^{1530 A}$
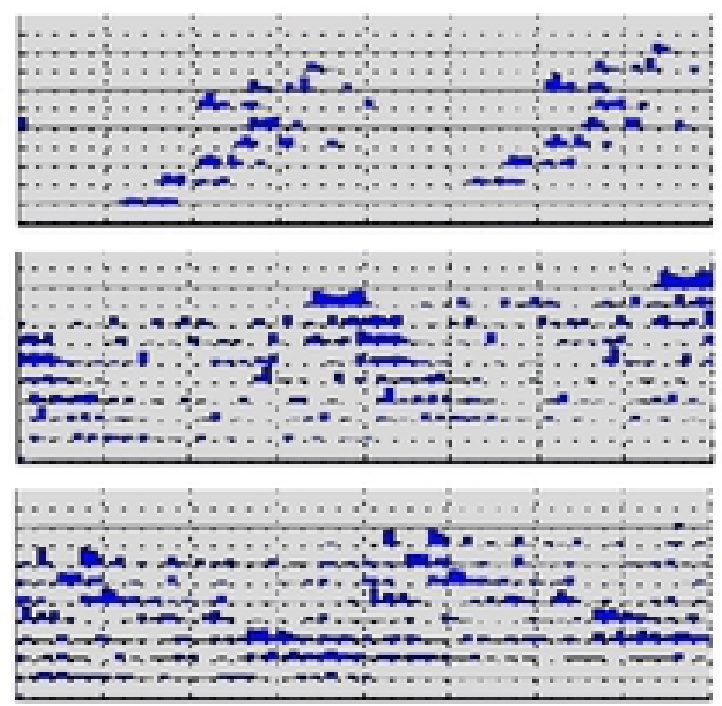

$\operatorname{per}^{15}$
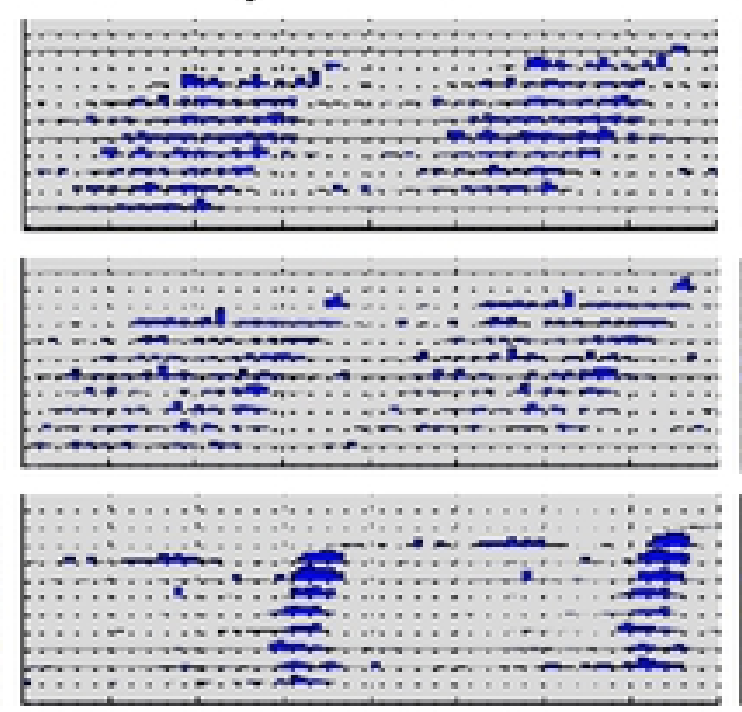

per ${ }^{1530 A-v 5}$

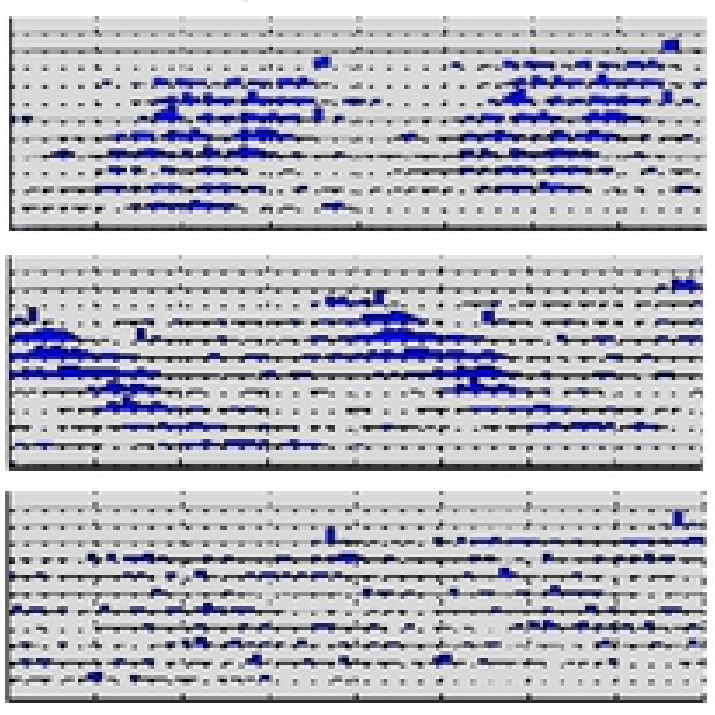

C
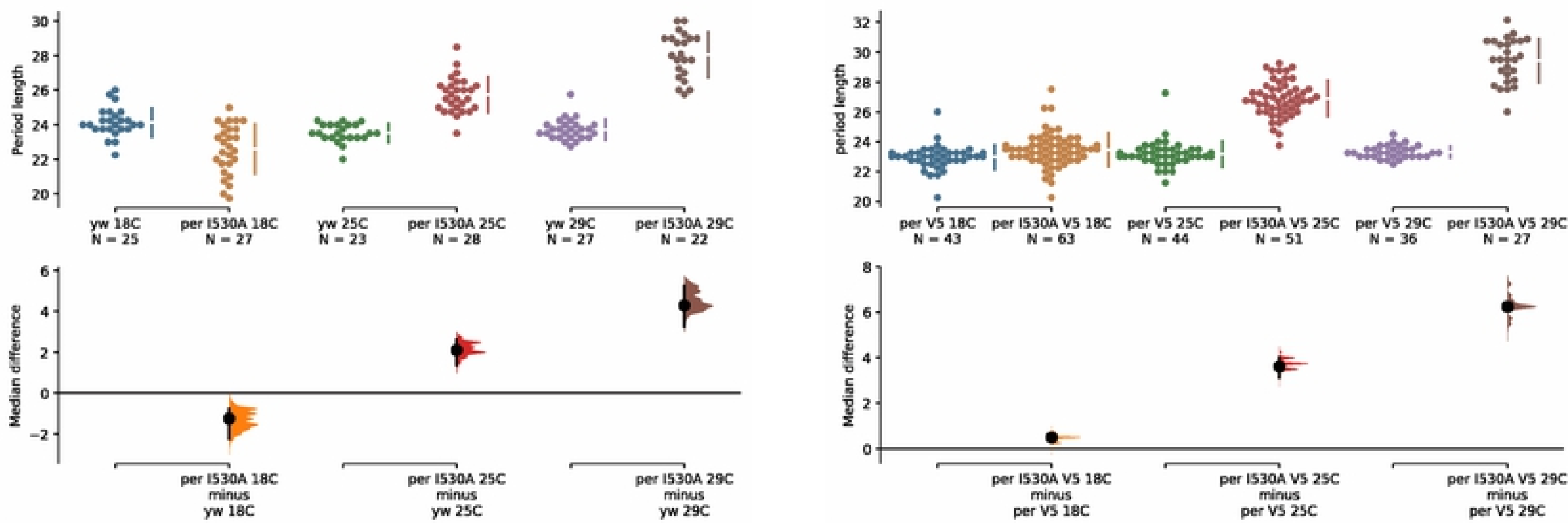

per IS30A v5 25C
minus

per 1530 A vs $29 \mathrm{C}$ minus
per V5 $29 \mathrm{C}$ 


\section{Figure 2}

A

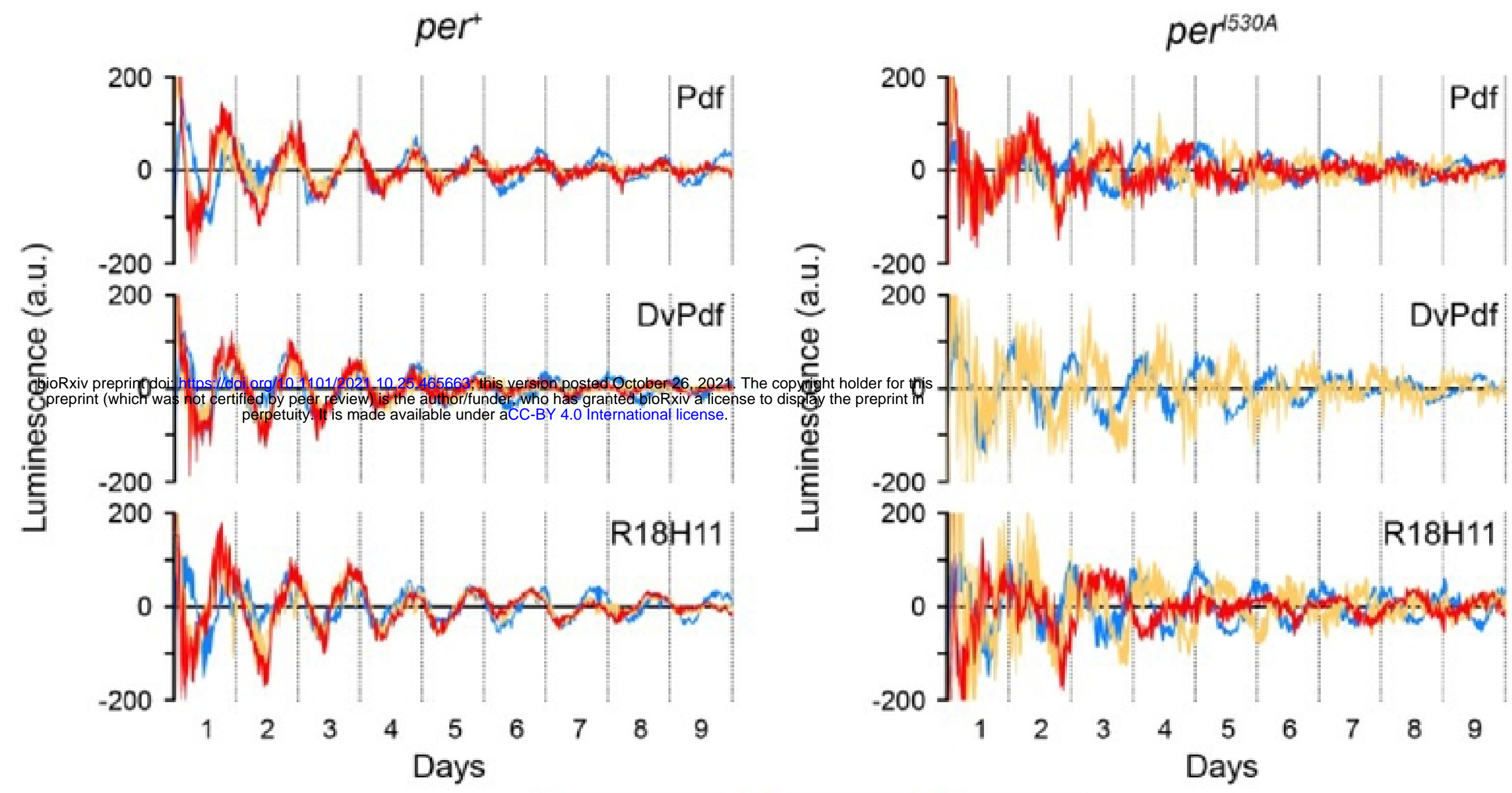

B

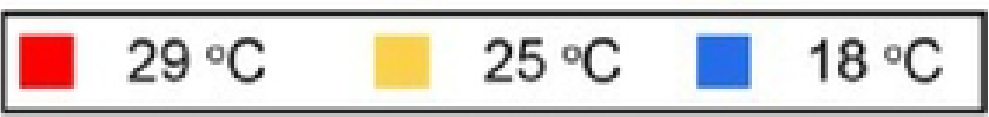

per

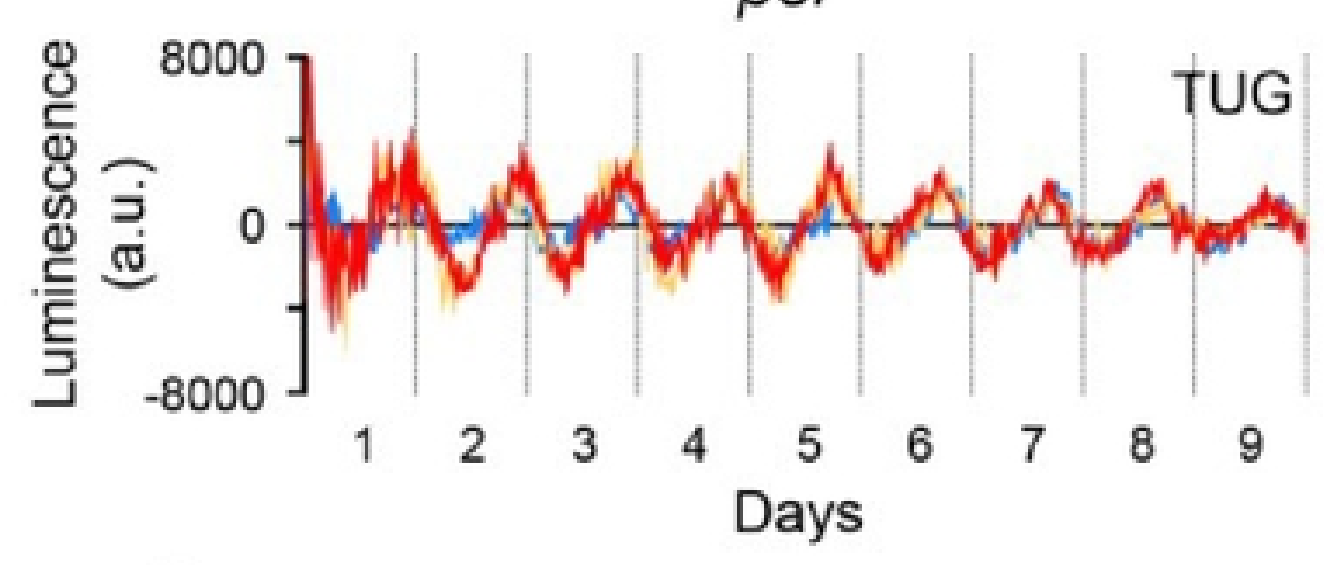

C

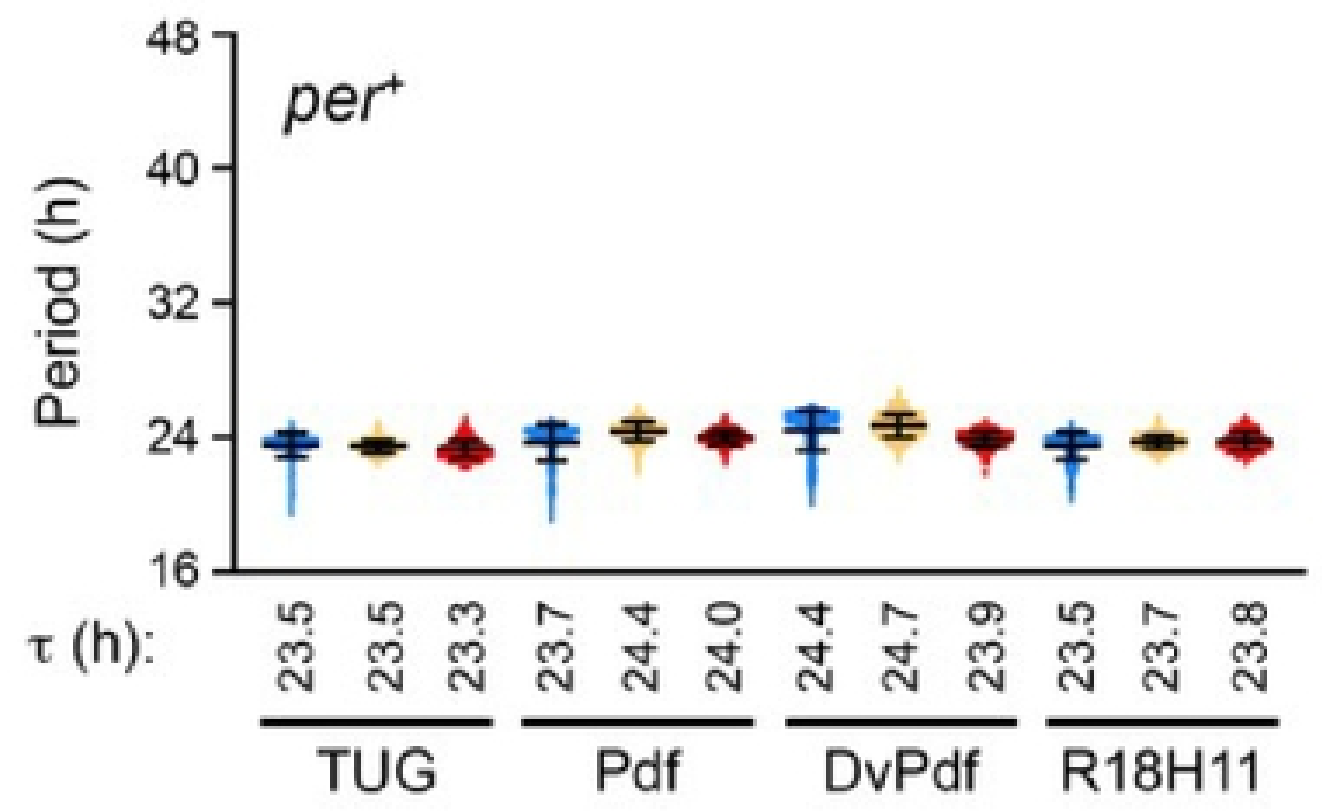

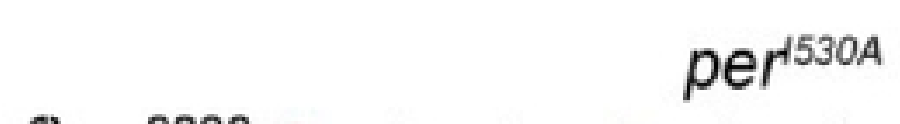
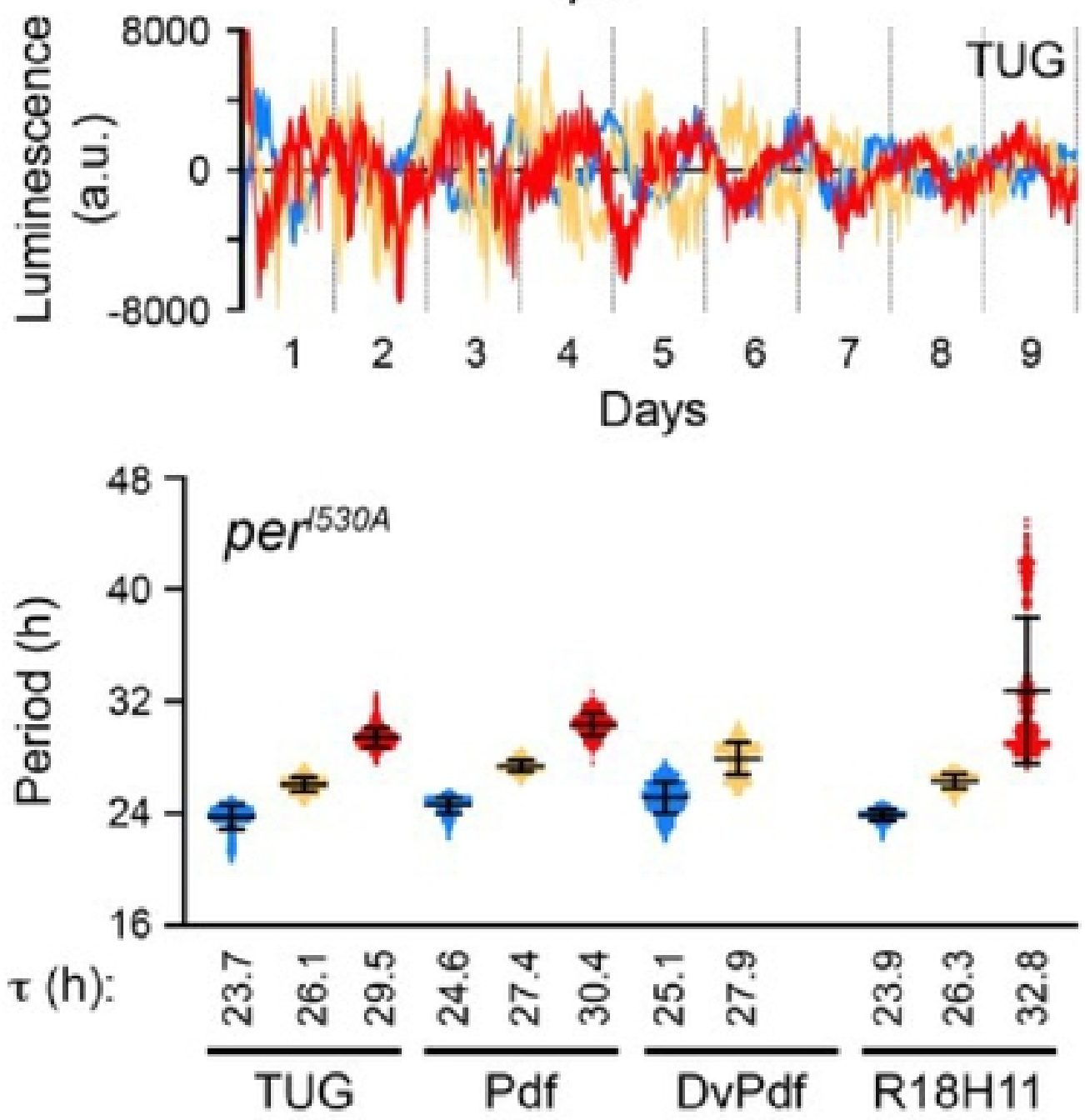
A

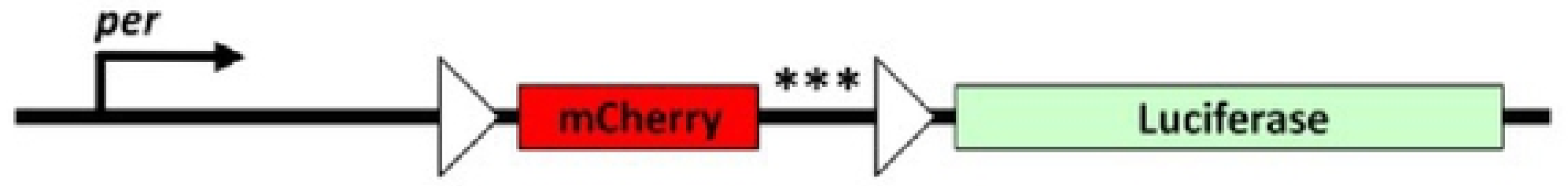

B

per

48

TUG

487

per ${ }^{1530 A}$

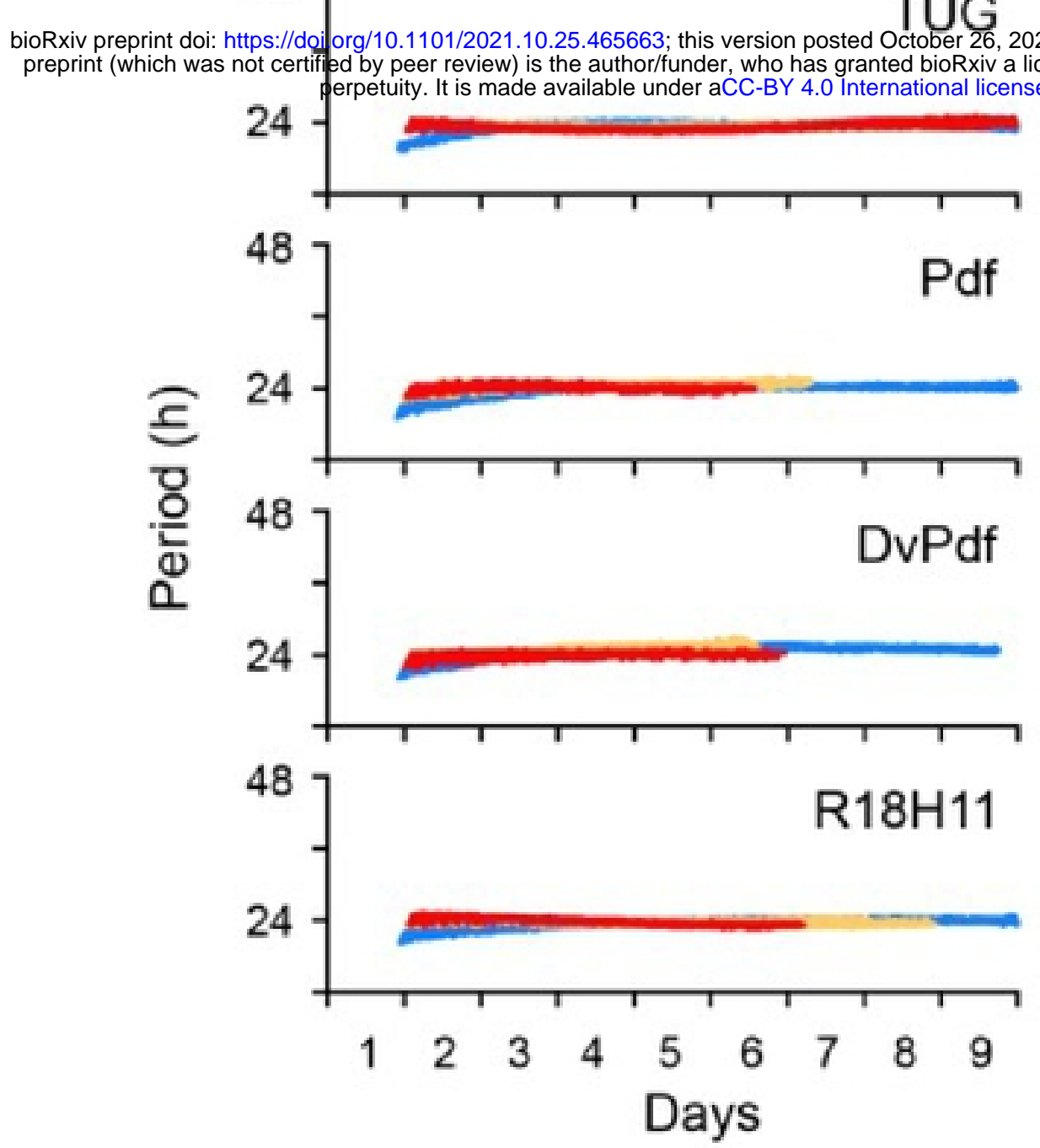

TUG

bioRxiv preprint doi: https://doilorg/10.1101/2021.10.25.465663; this version posted October 26, 2021. The copyright holder for this
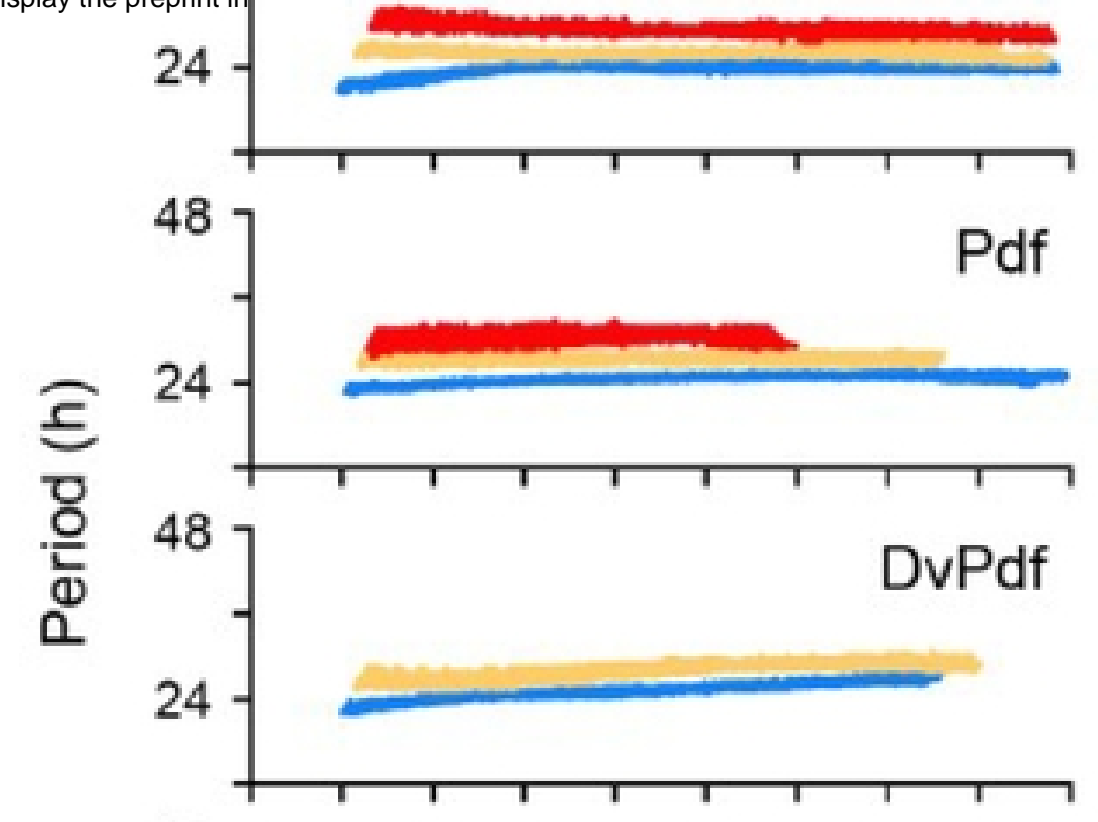

C

48

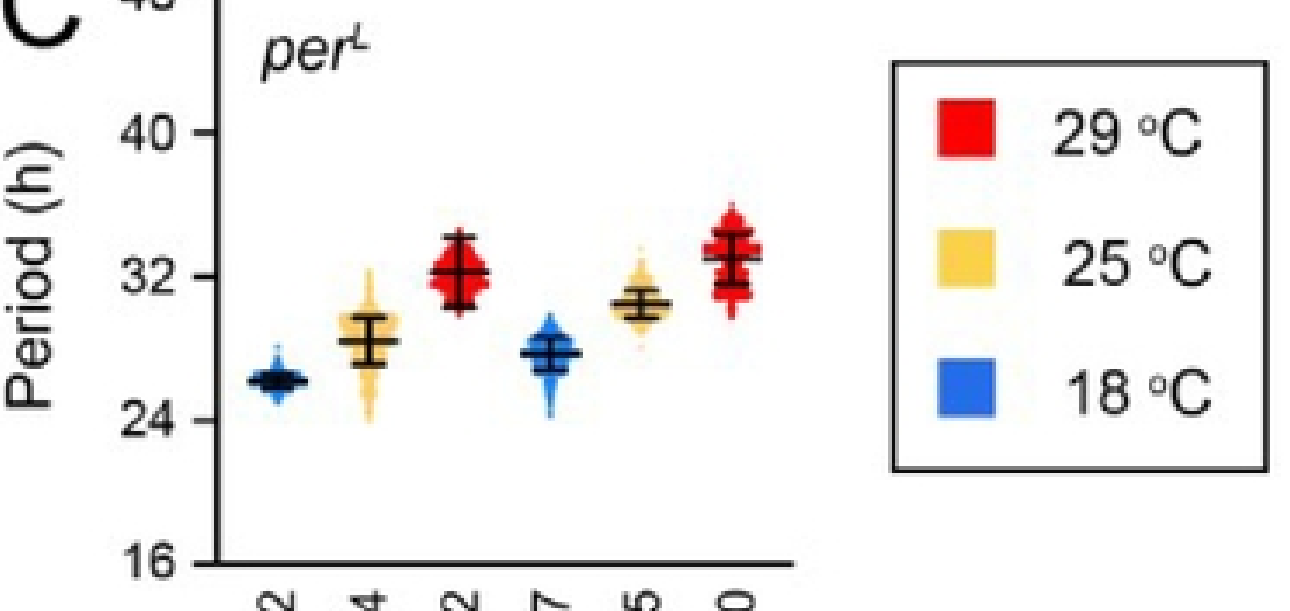

กู่

$\frac{\text { TUG }}{\text { Pdf }}$

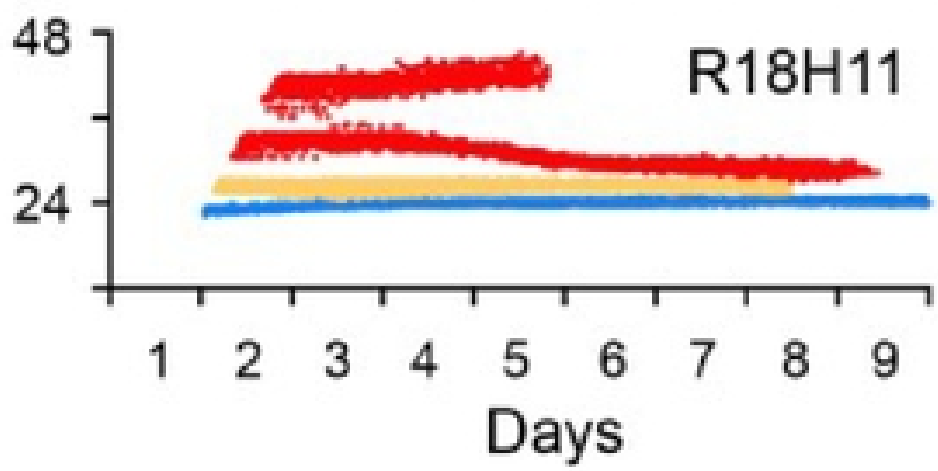

Days 
Figure 3

A
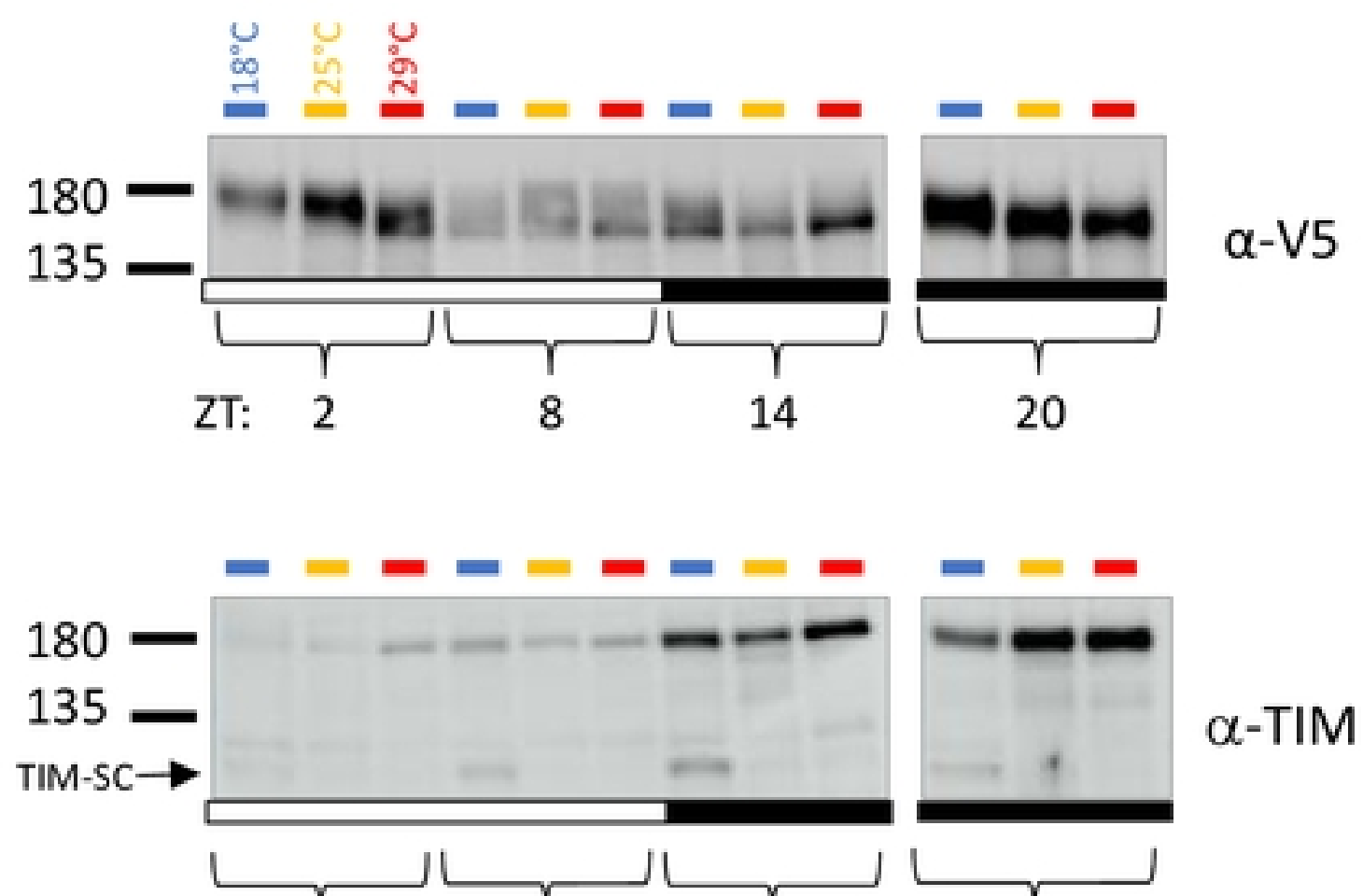

bioRxiv preprint doi: https://doi.org/10.1101/2021.10.25.465663; this version posted October 26, 2021. The copyright holder for this preprint (whijeh was 2 ot certified by peerceview) is the authorfunder, who has granted bioRxiv a license to display the preprint in
perpetuity. \$is made available thder aCC-BY 4.0 Interdational license.

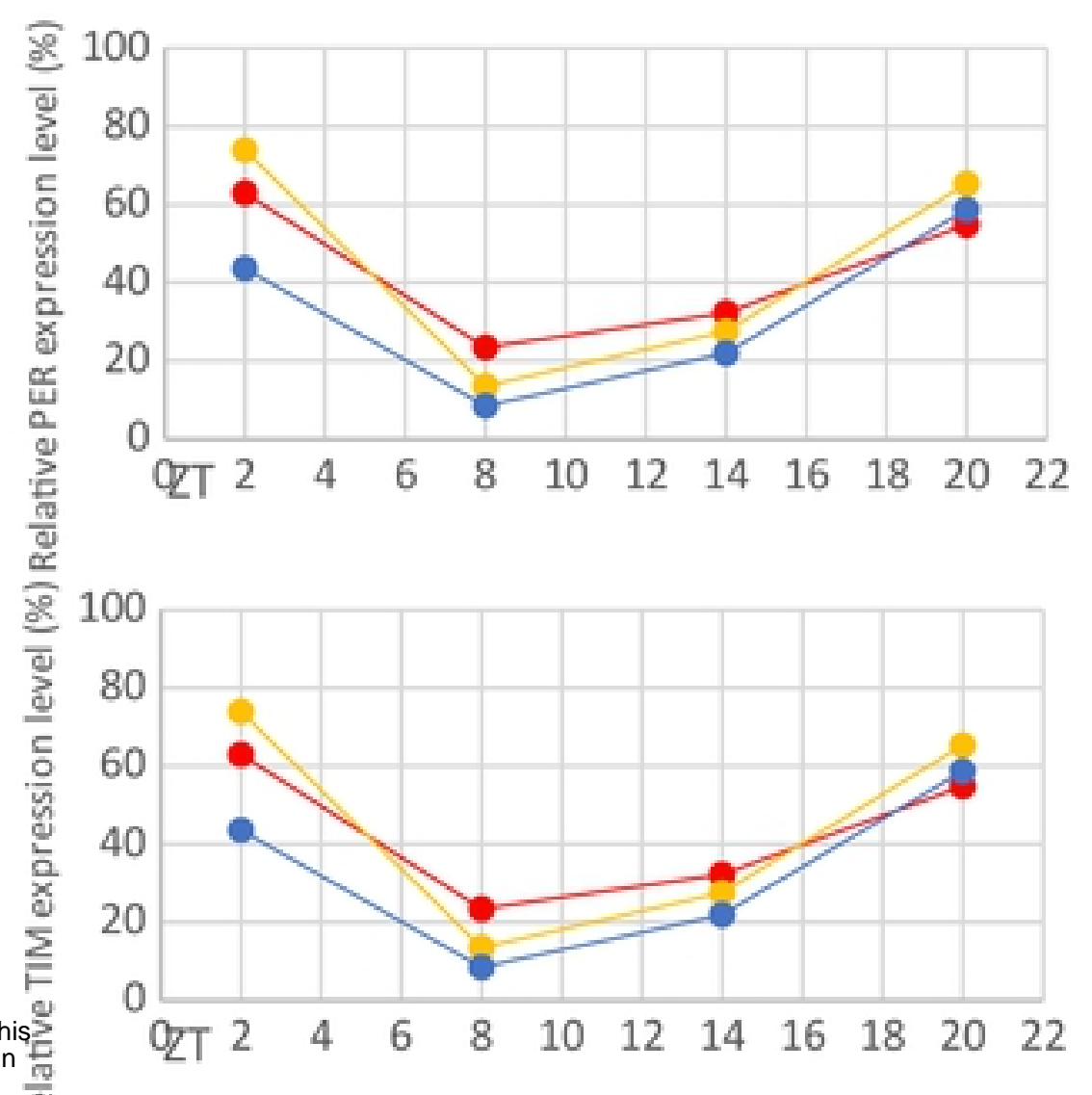

B
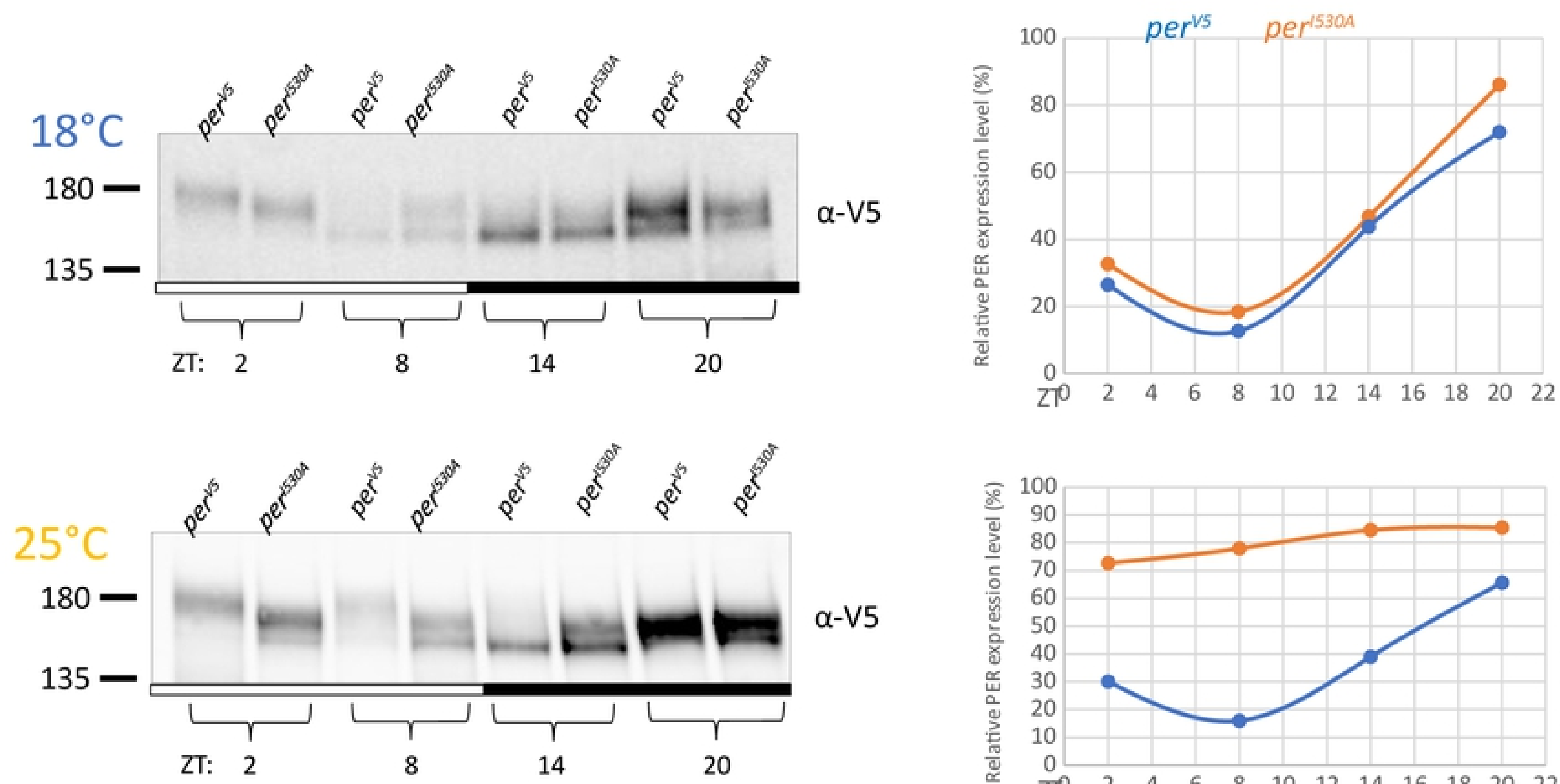

$\alpha-\mathrm{V} 5$
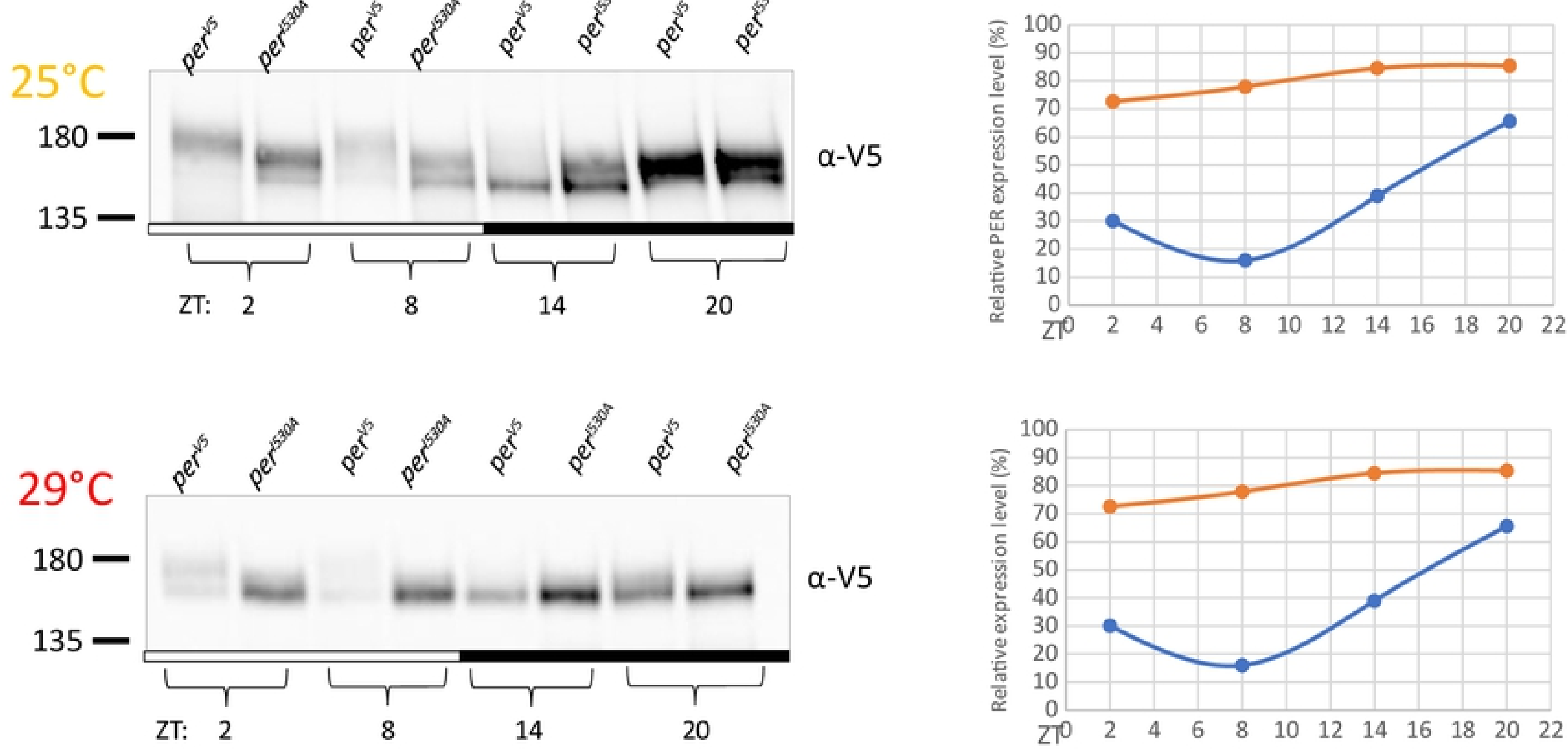
Figure S2

$18^{\circ} \mathrm{C}$

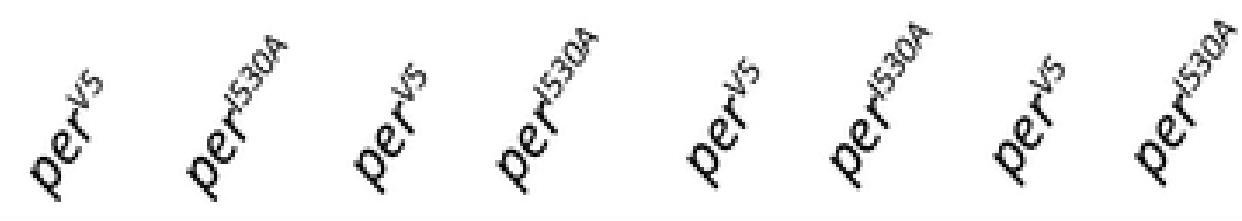

${ }_{\alpha . T M}$
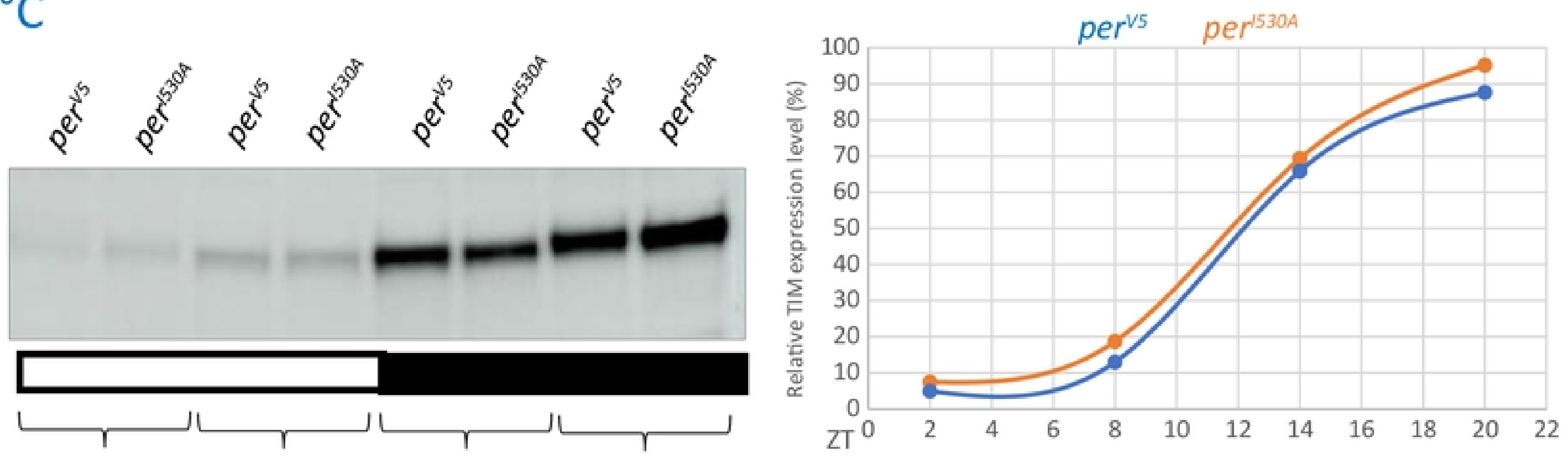

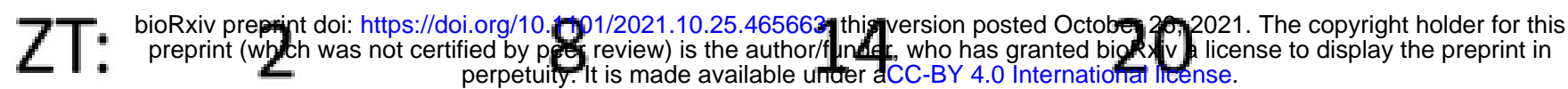

$25^{\circ} \mathrm{C}$

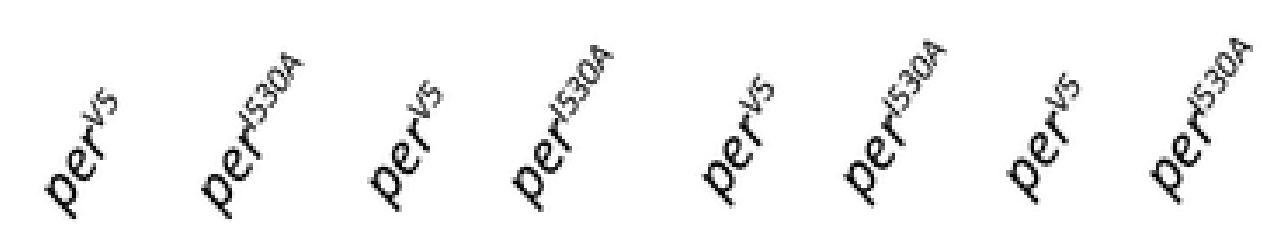

$\alpha-$ TIM

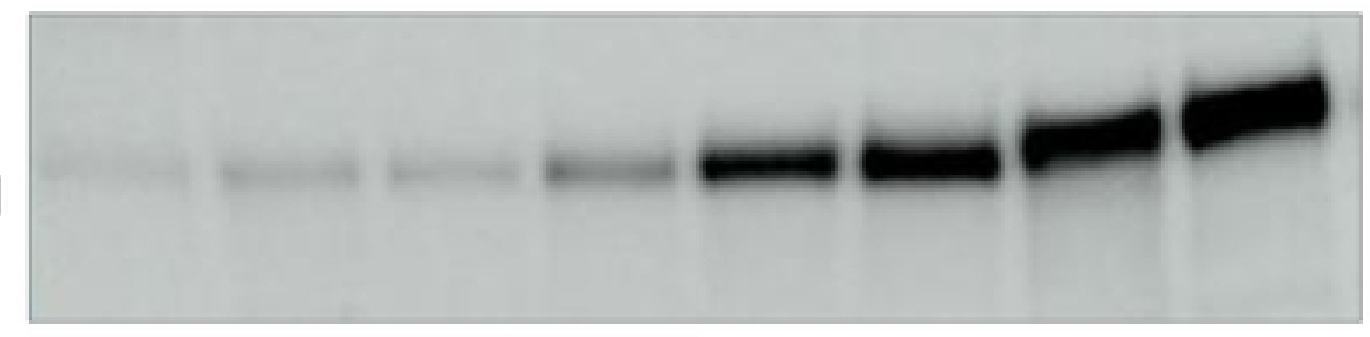

$29^{\circ} \mathrm{C}$
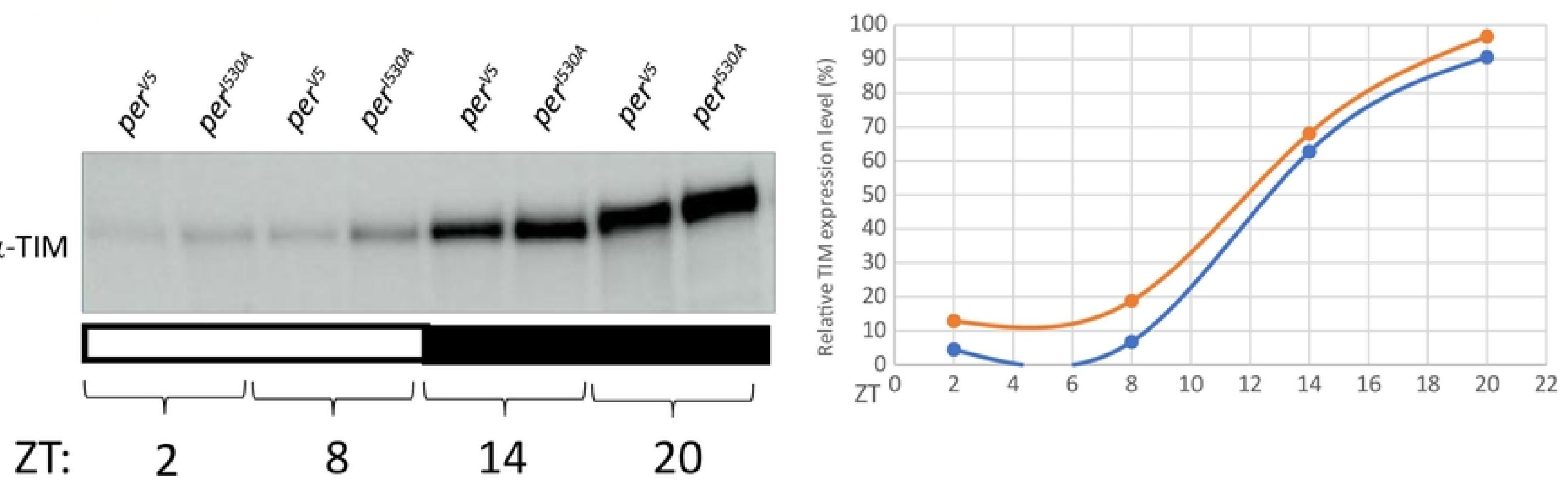

$\alpha-\mathrm{TIM}$

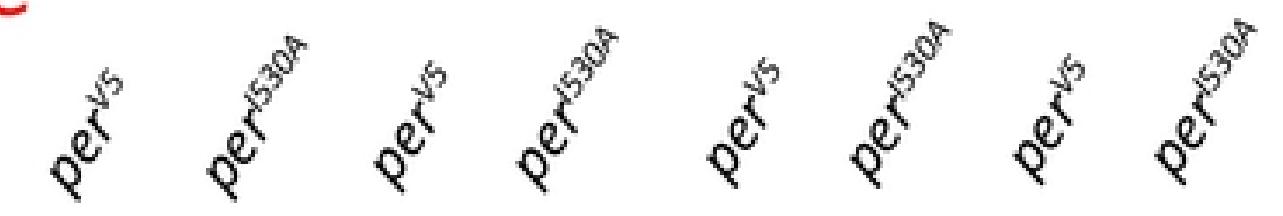

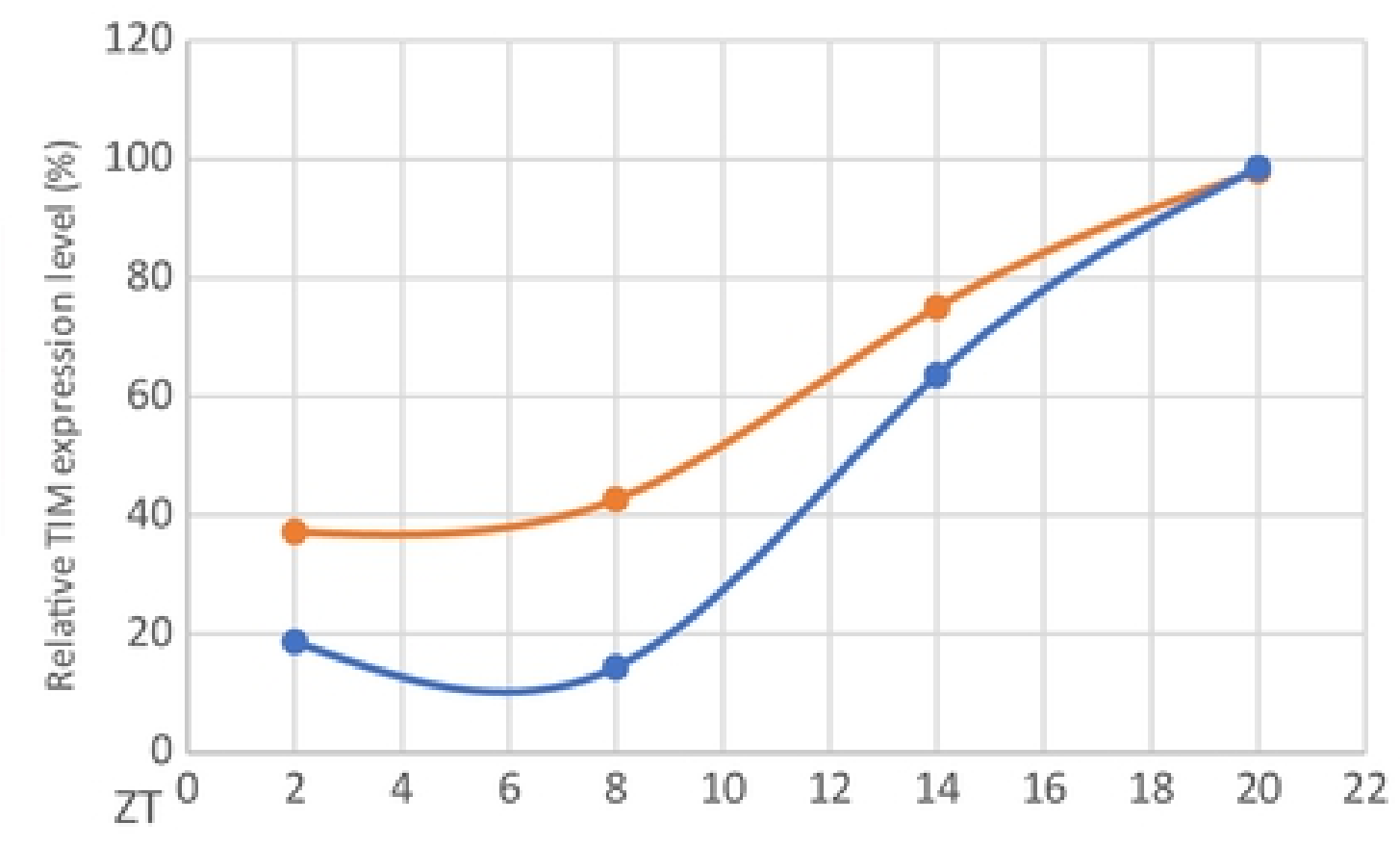




\section{Figure S3}

$18^{\circ} \mathrm{C}$

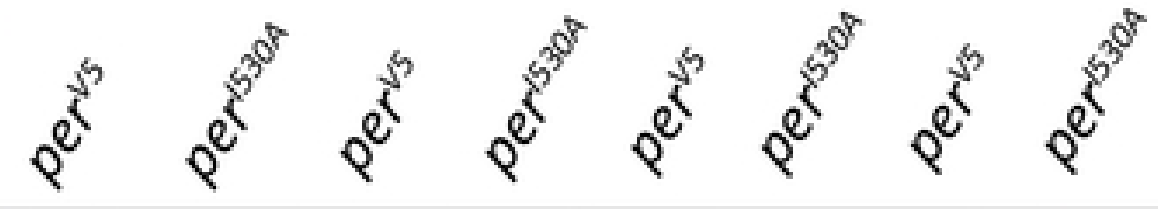

$\alpha$-PER

$\alpha$-TIM

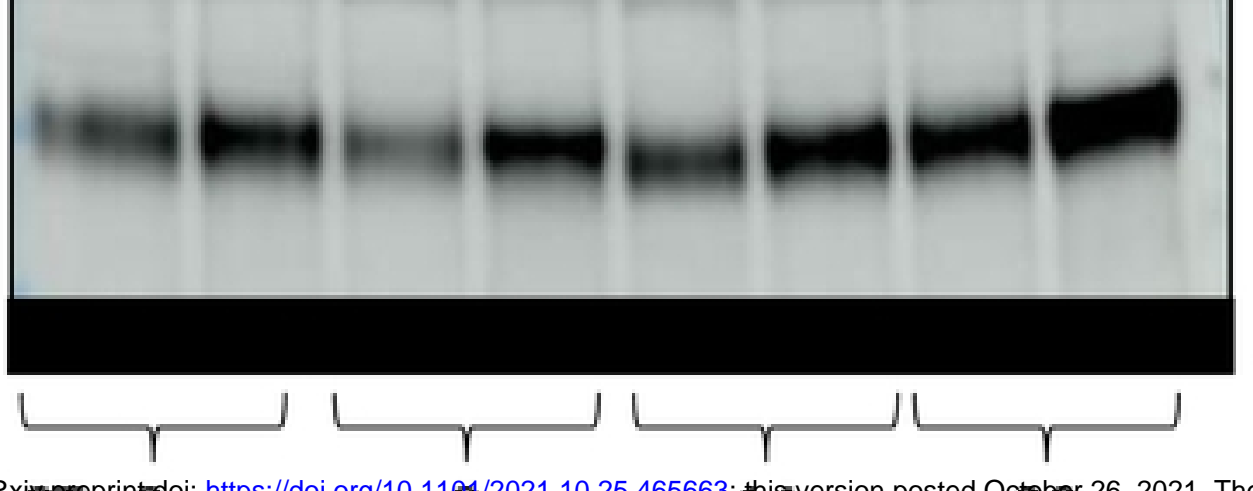

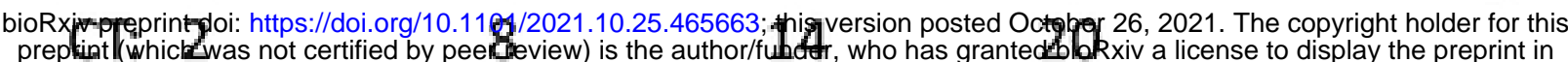
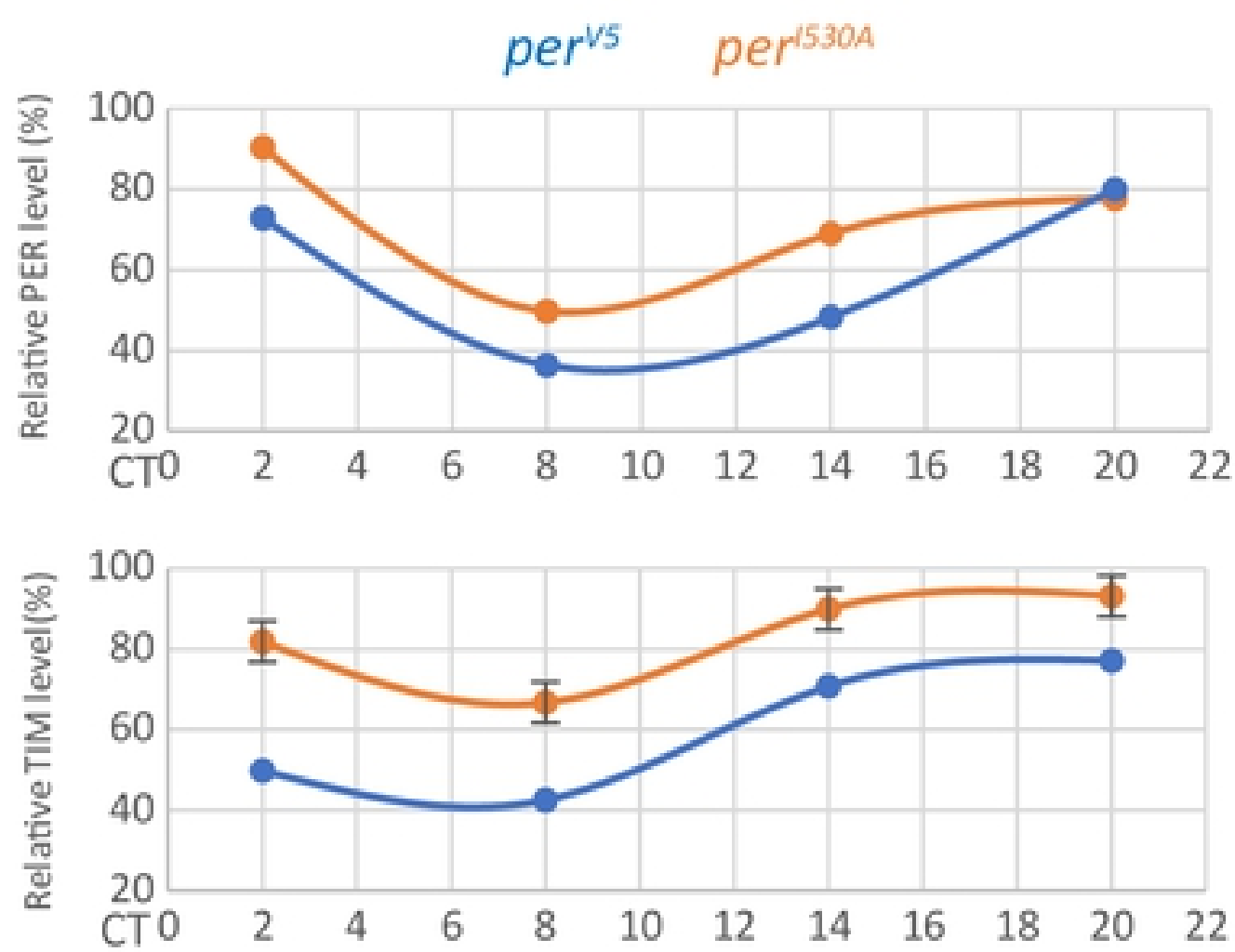
perpetuity. It is made available under aCC-BY 4.0 International license.

$25^{\circ} \mathrm{C}$
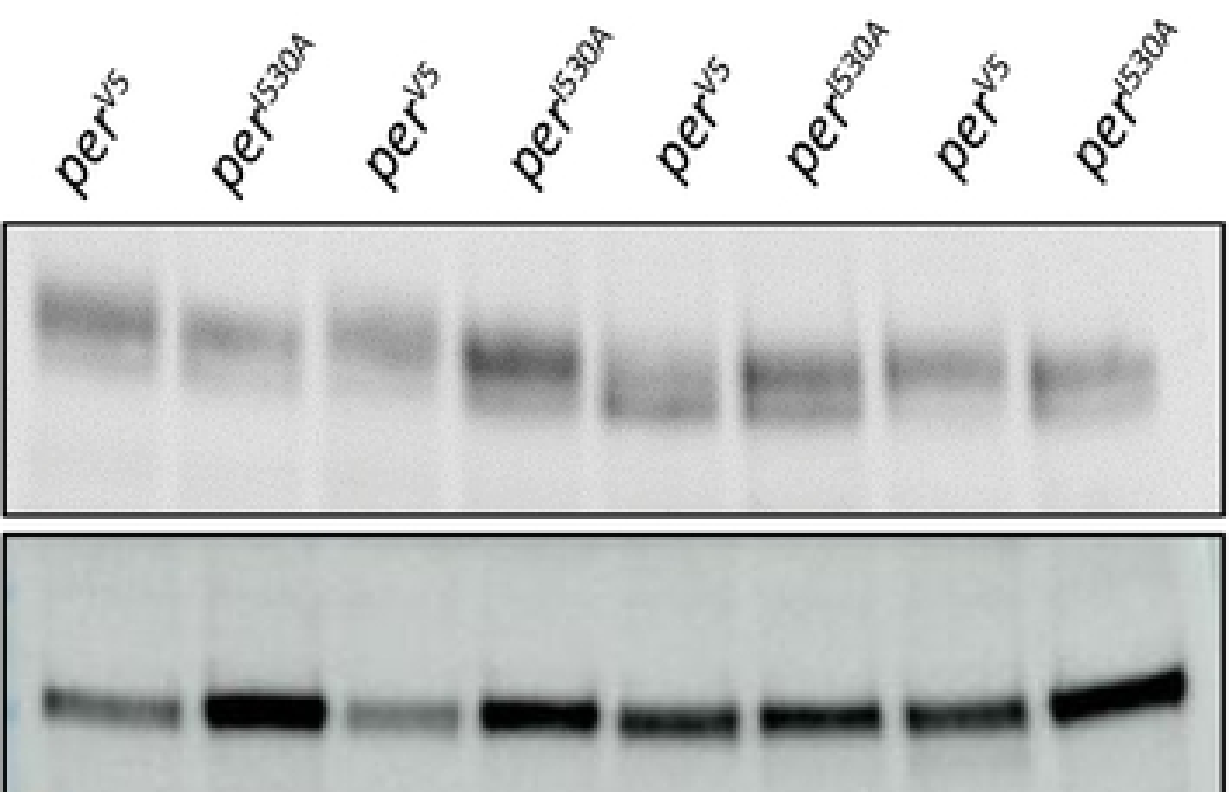

$29^{\circ} \mathrm{C}$
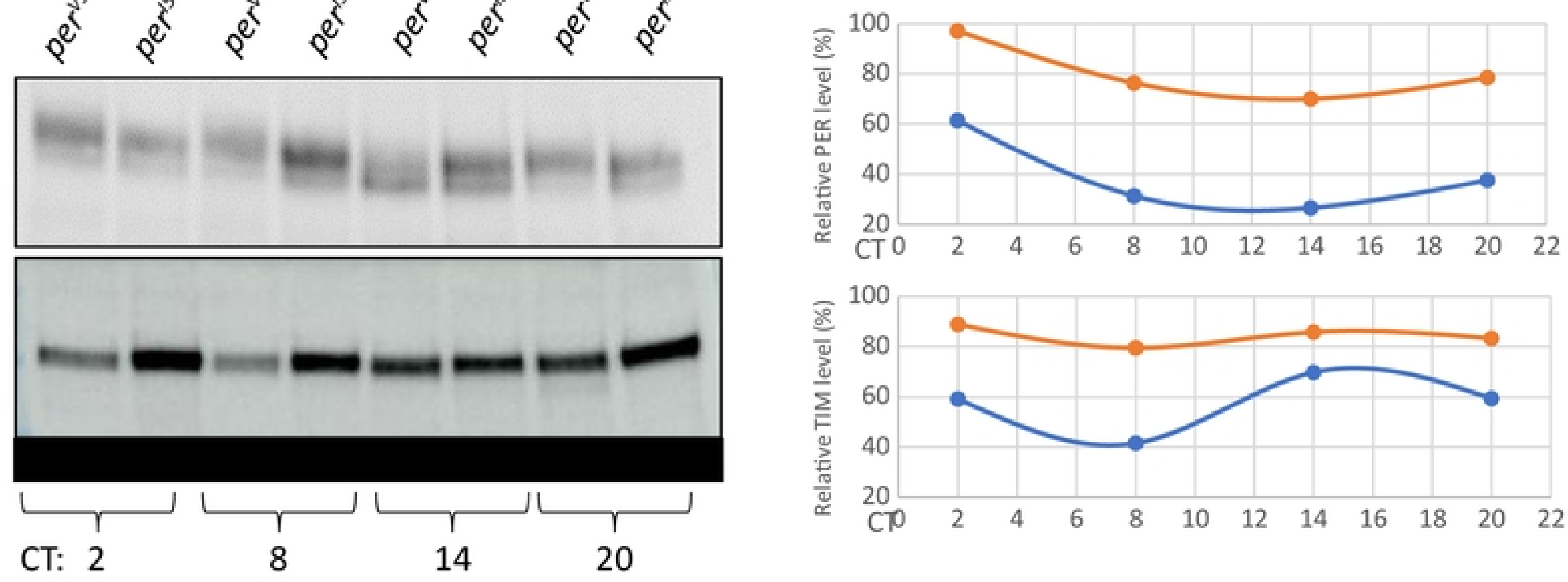

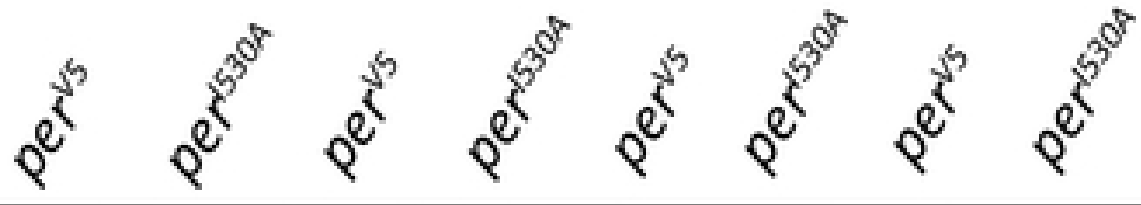

$\alpha$-TIM
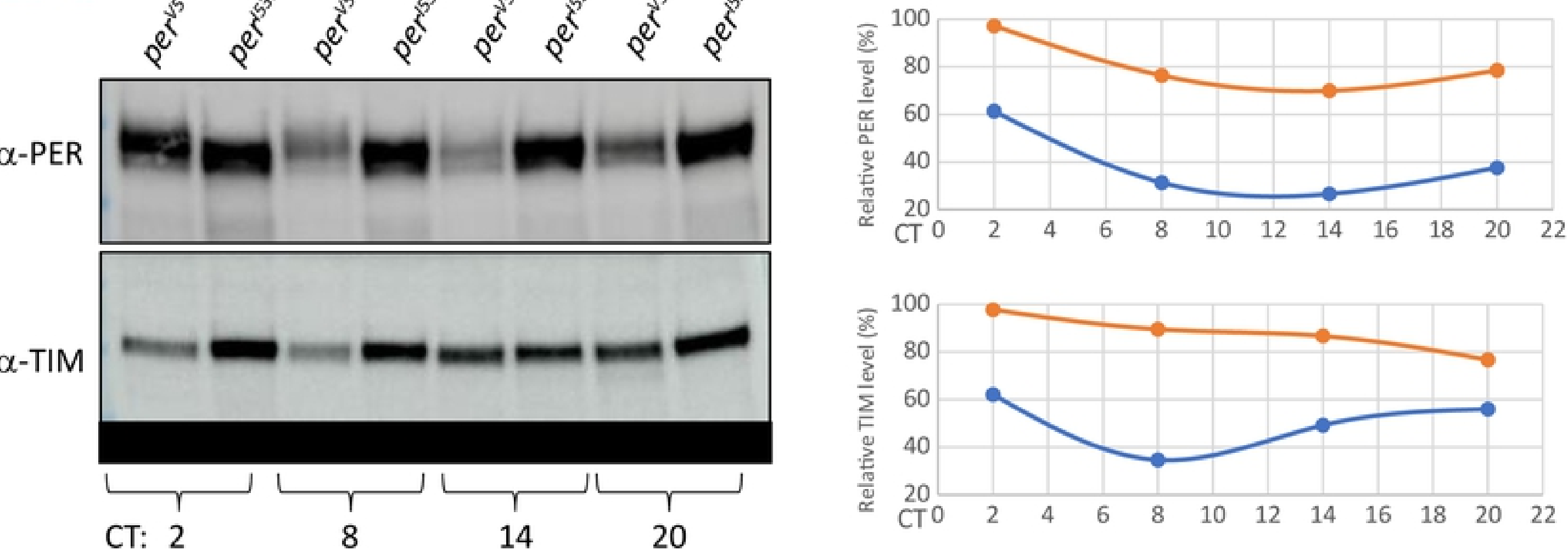
A
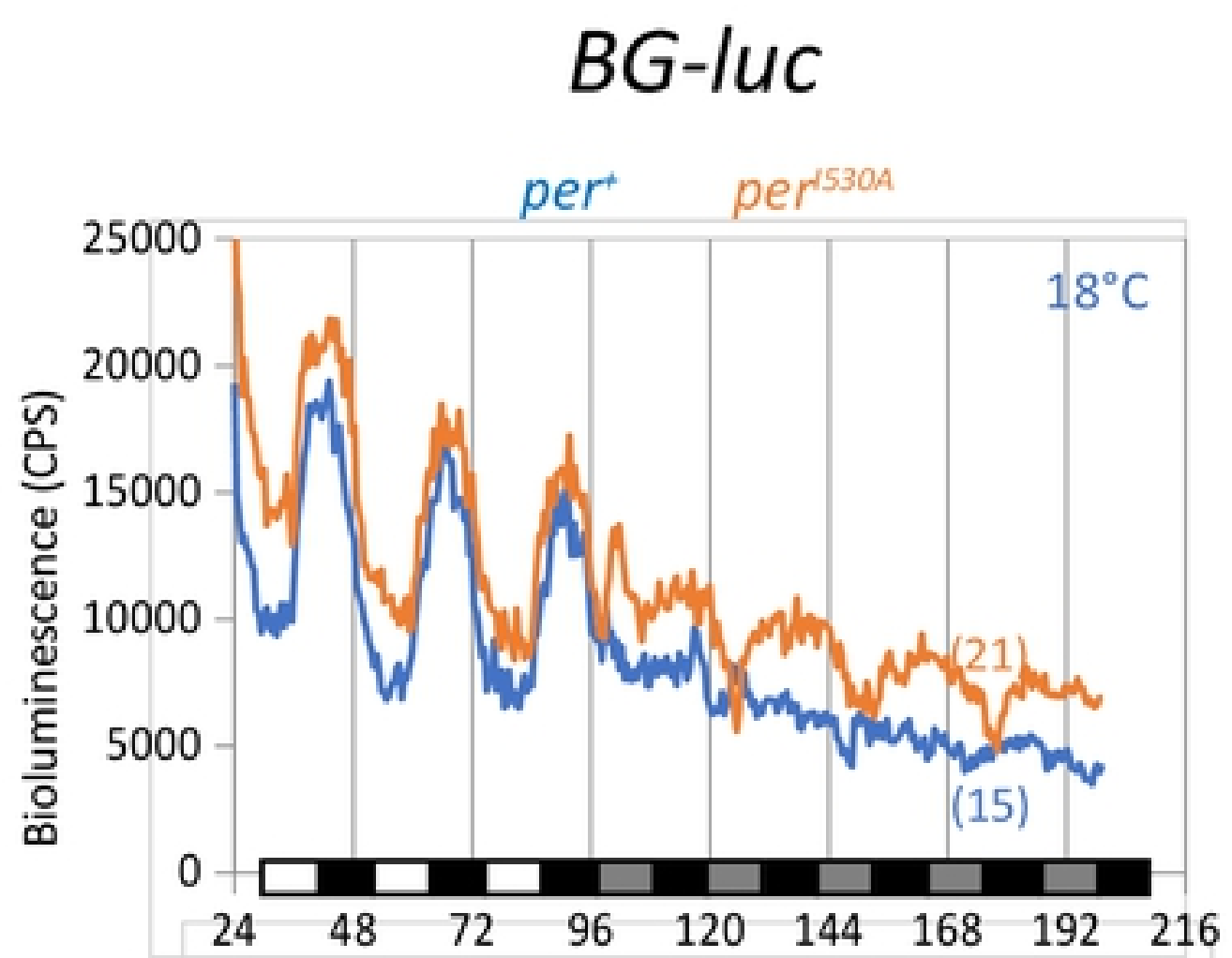

bioRxiv preprint doi: https://doi.org/10.1101/2021.10.25.465663; this version posted October 26, 2021.
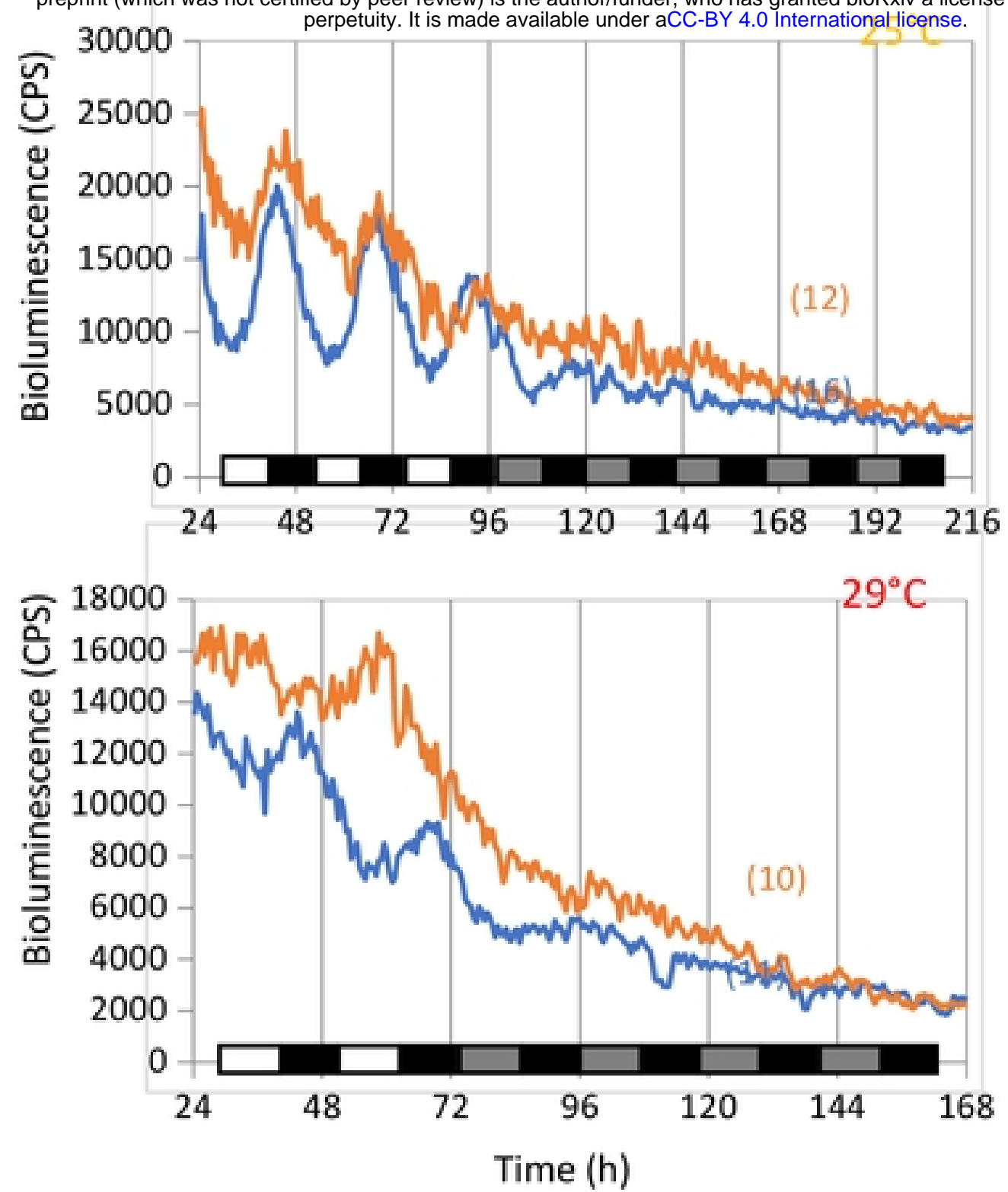

C

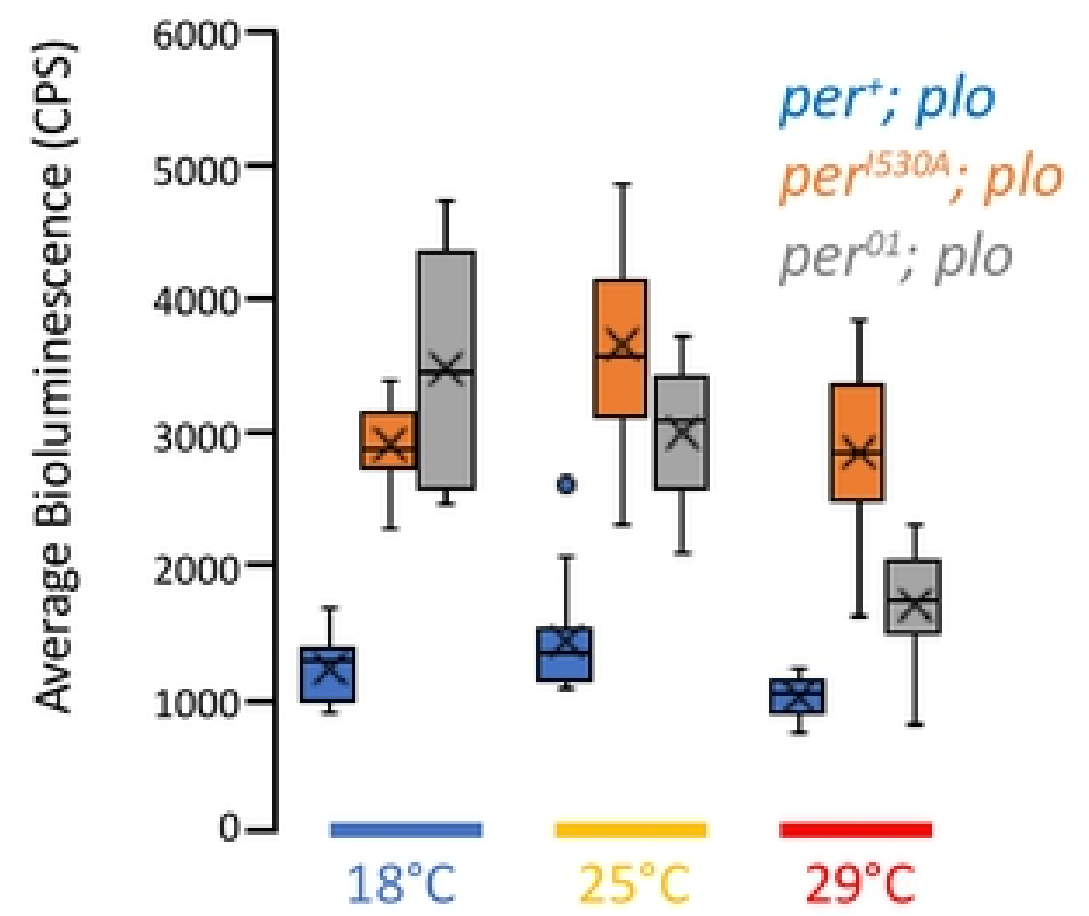

B

plo

per $^{+}$peris304 per ${ }^{01}$
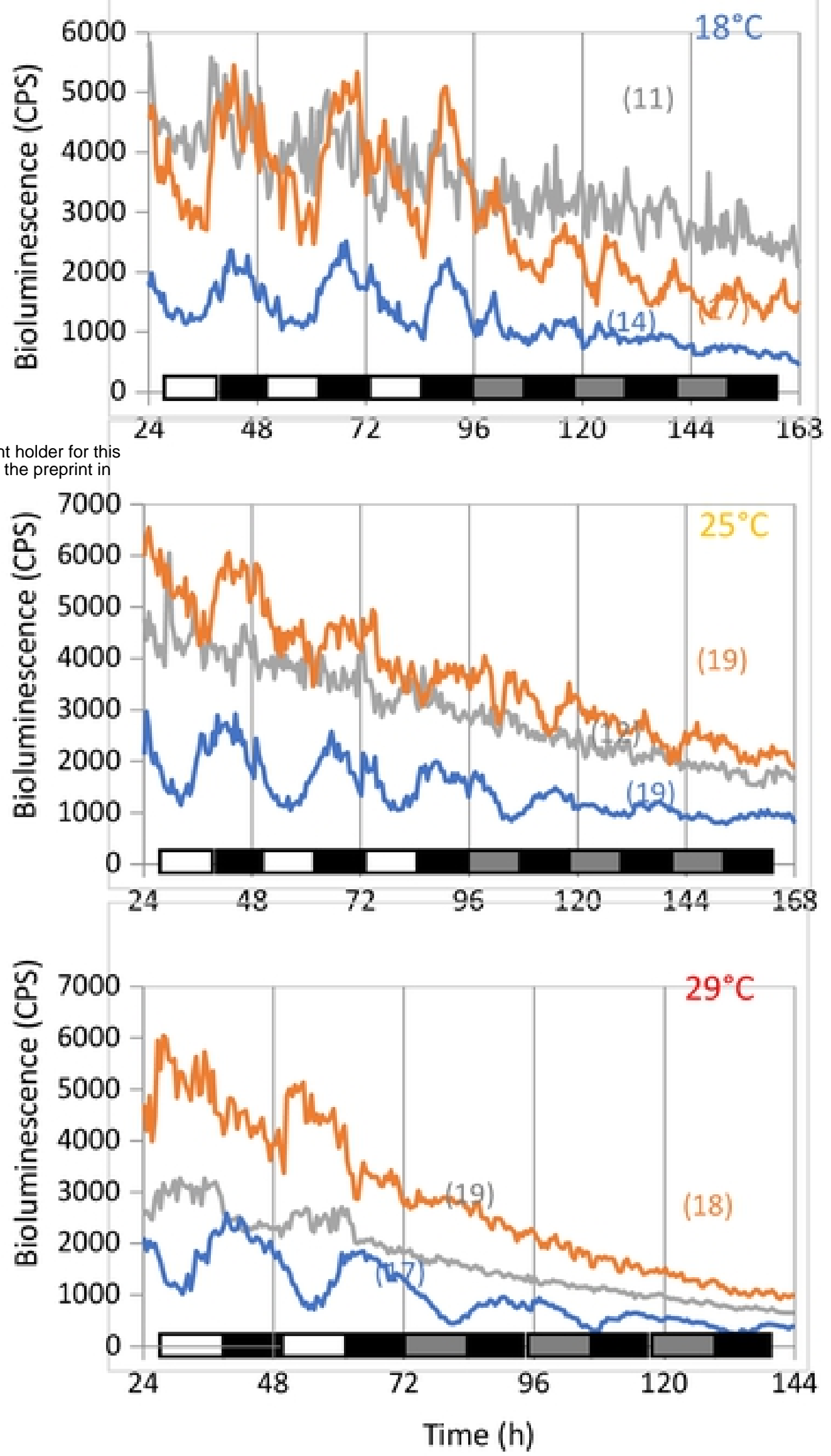

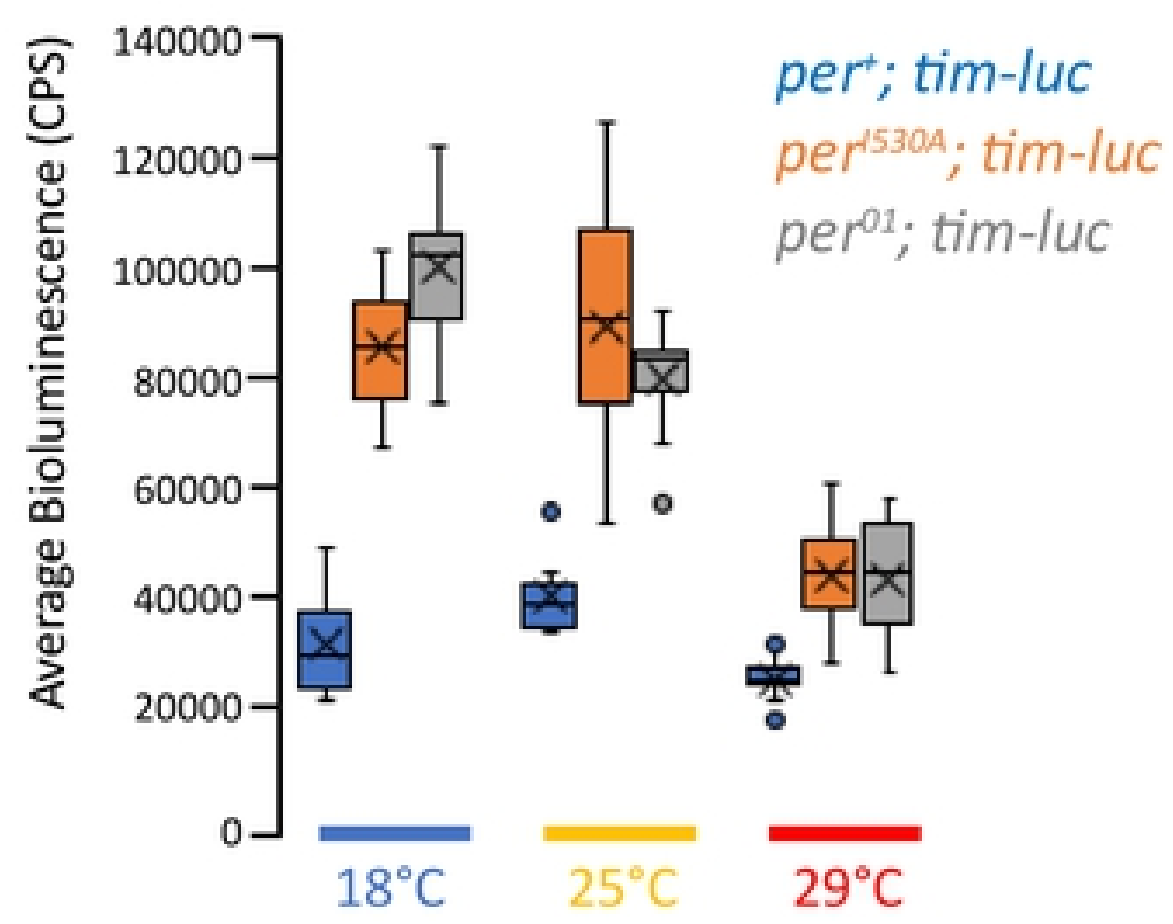




\section{Figure S4}

A
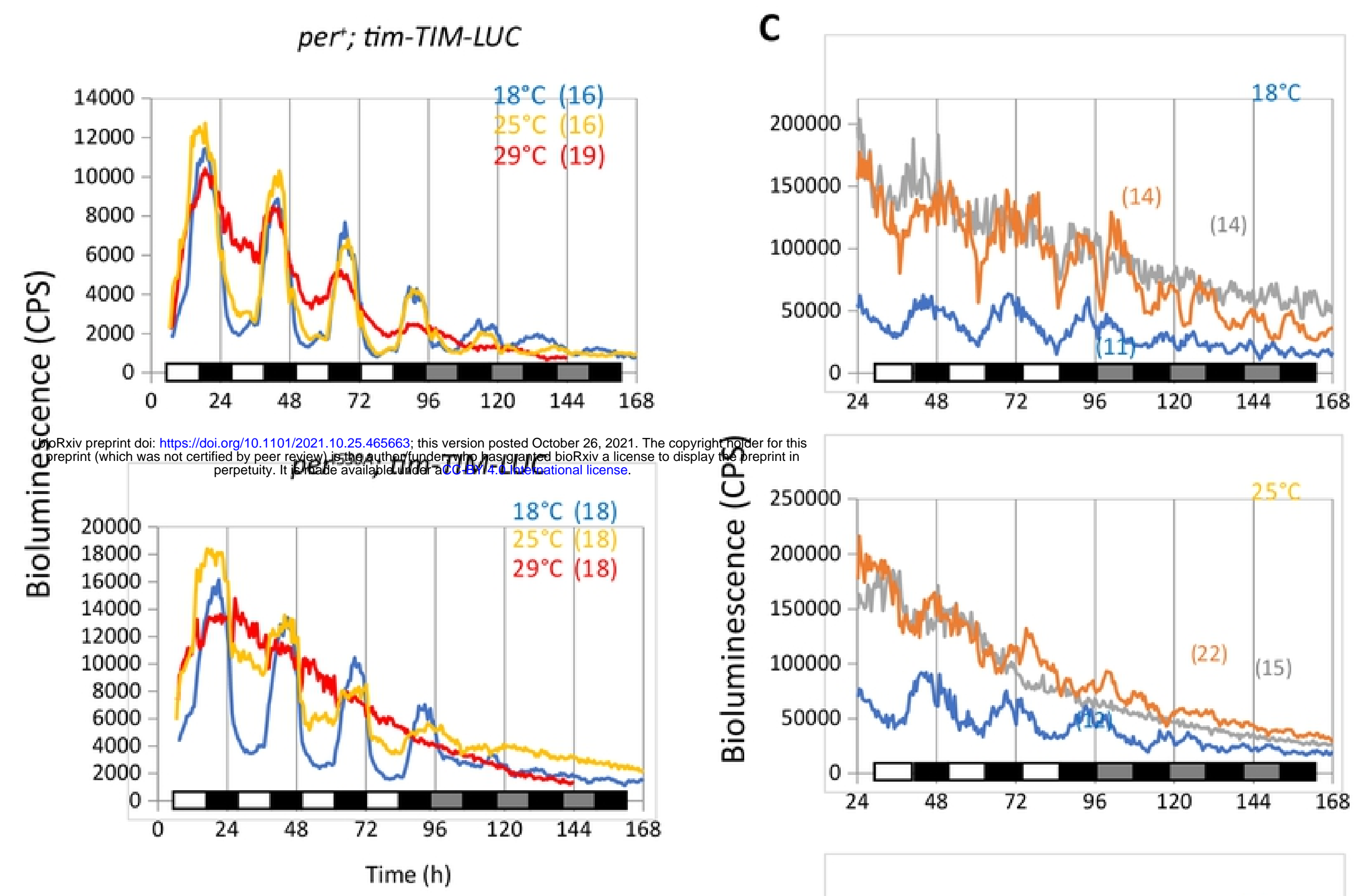

B
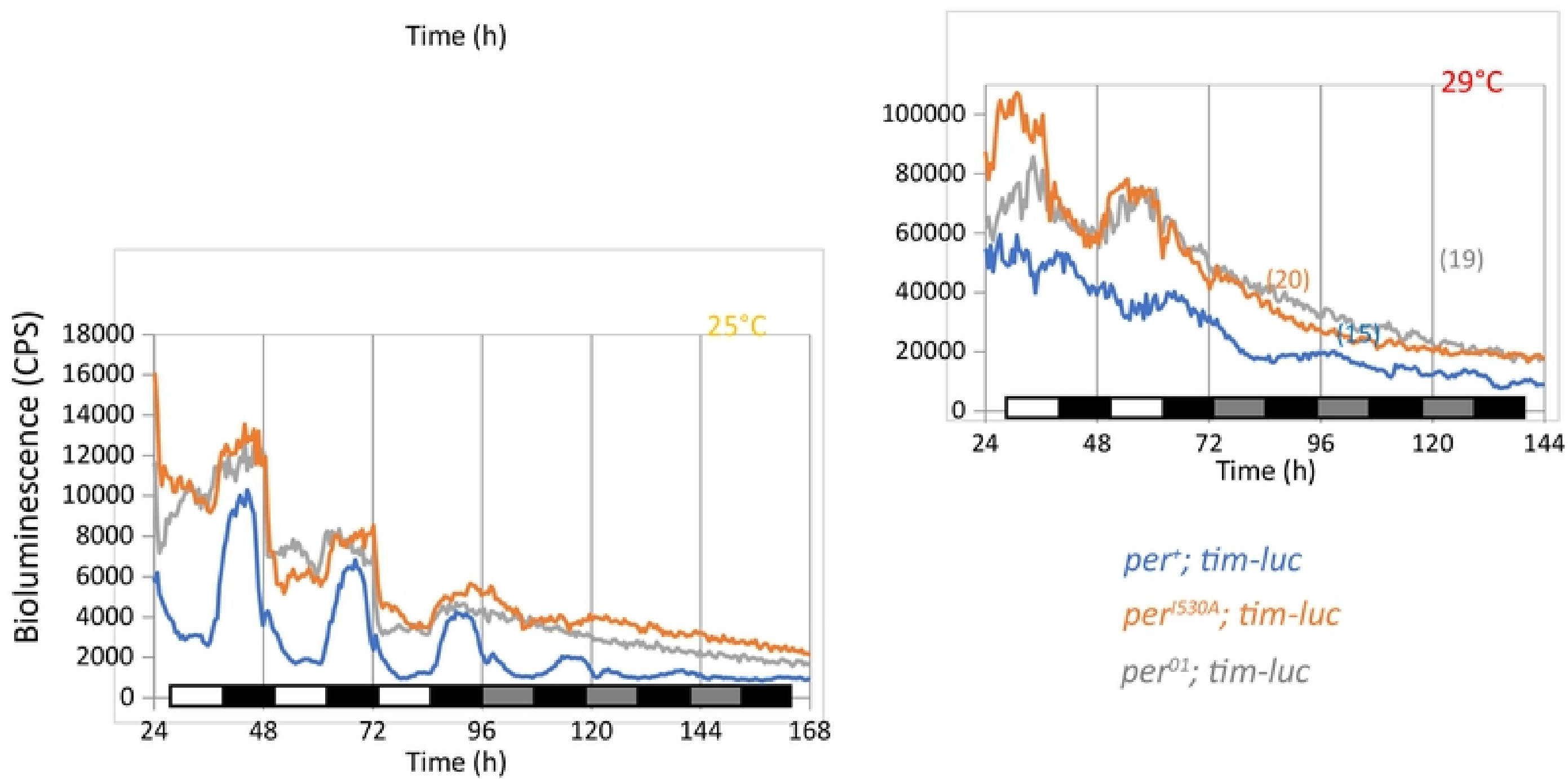

per'; tim-TIM-LUC ( $\mathrm{n}=16)$

per ${ }^{1530 A}$; tim-TIM-LUC $(\mathrm{n}=18)$

per ${ }^{01}$; tim-TIM-LUC $(\mathrm{n}=6)$

pert; tim-luc

peri530A; tim-luc

pero1; tim-luc 
Figure 5

A
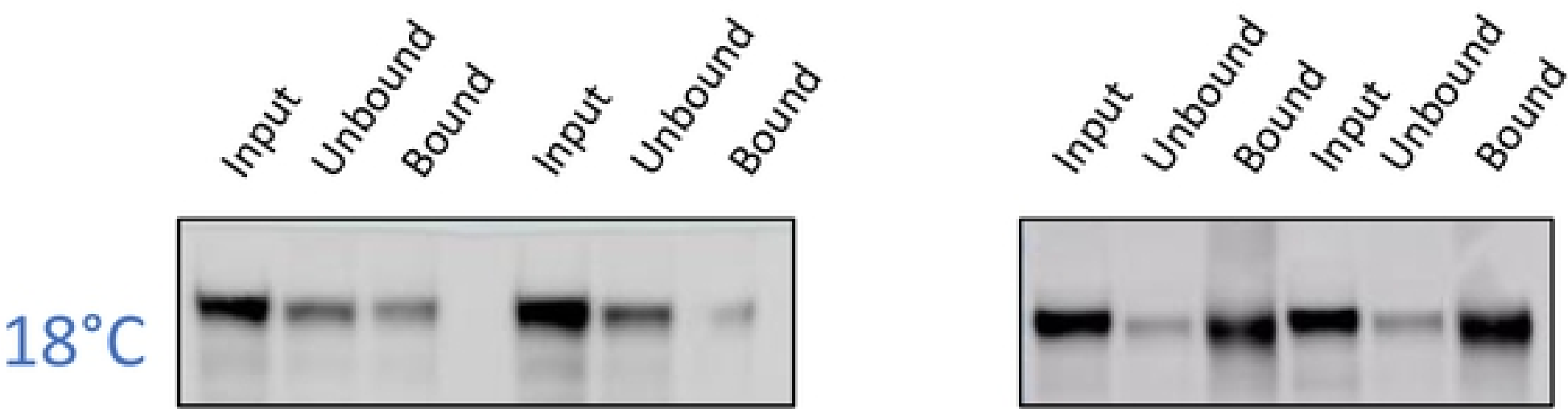

$\alpha-$ TIM

$25^{\circ} \mathrm{C}$
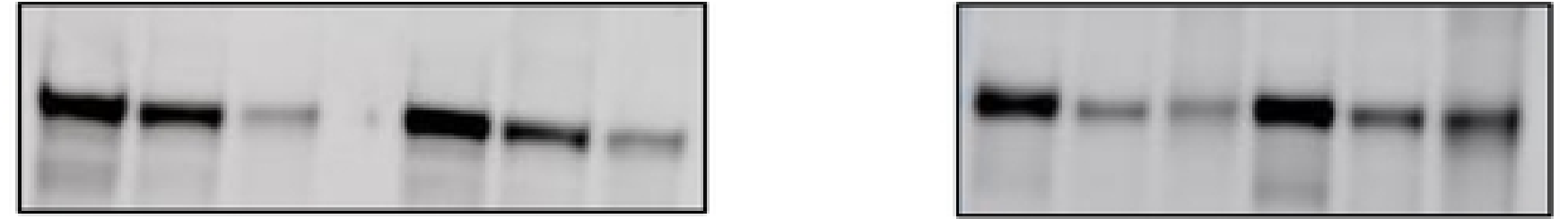

$\alpha$-TIM

$20^{\circ}$

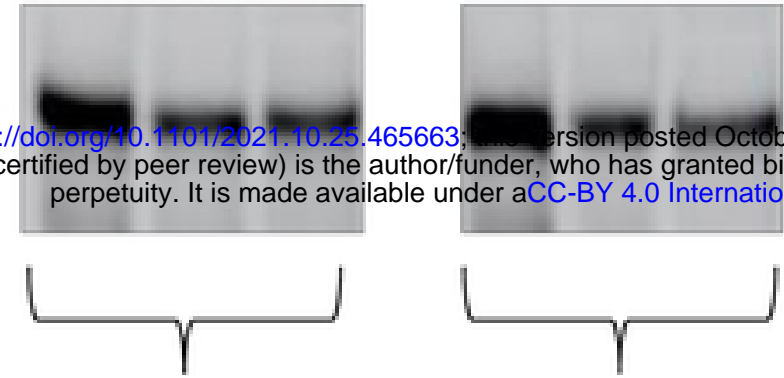

perv5

per 15304

per $^{\text {V5 }} \quad$ per $^{1530 A}$
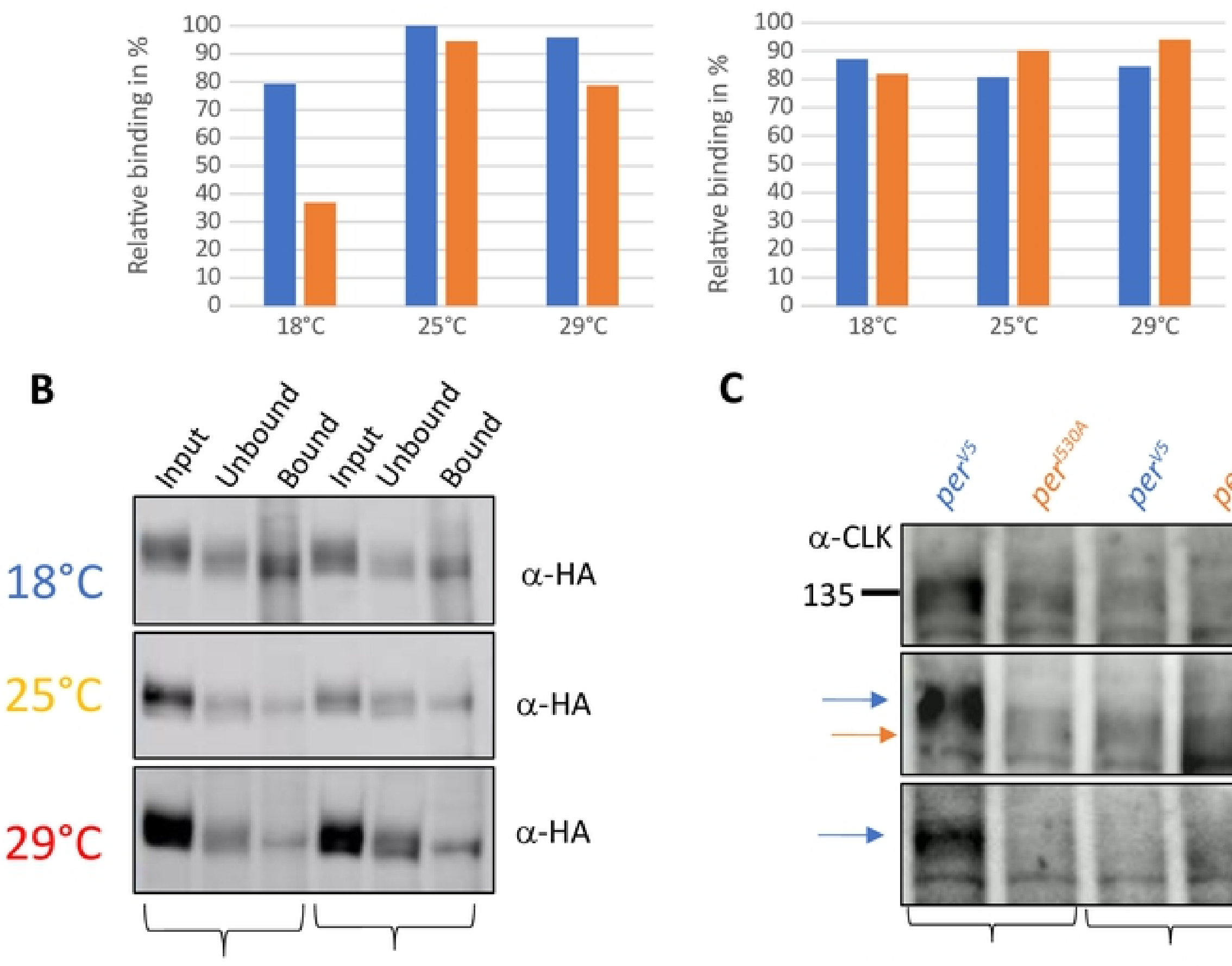

C
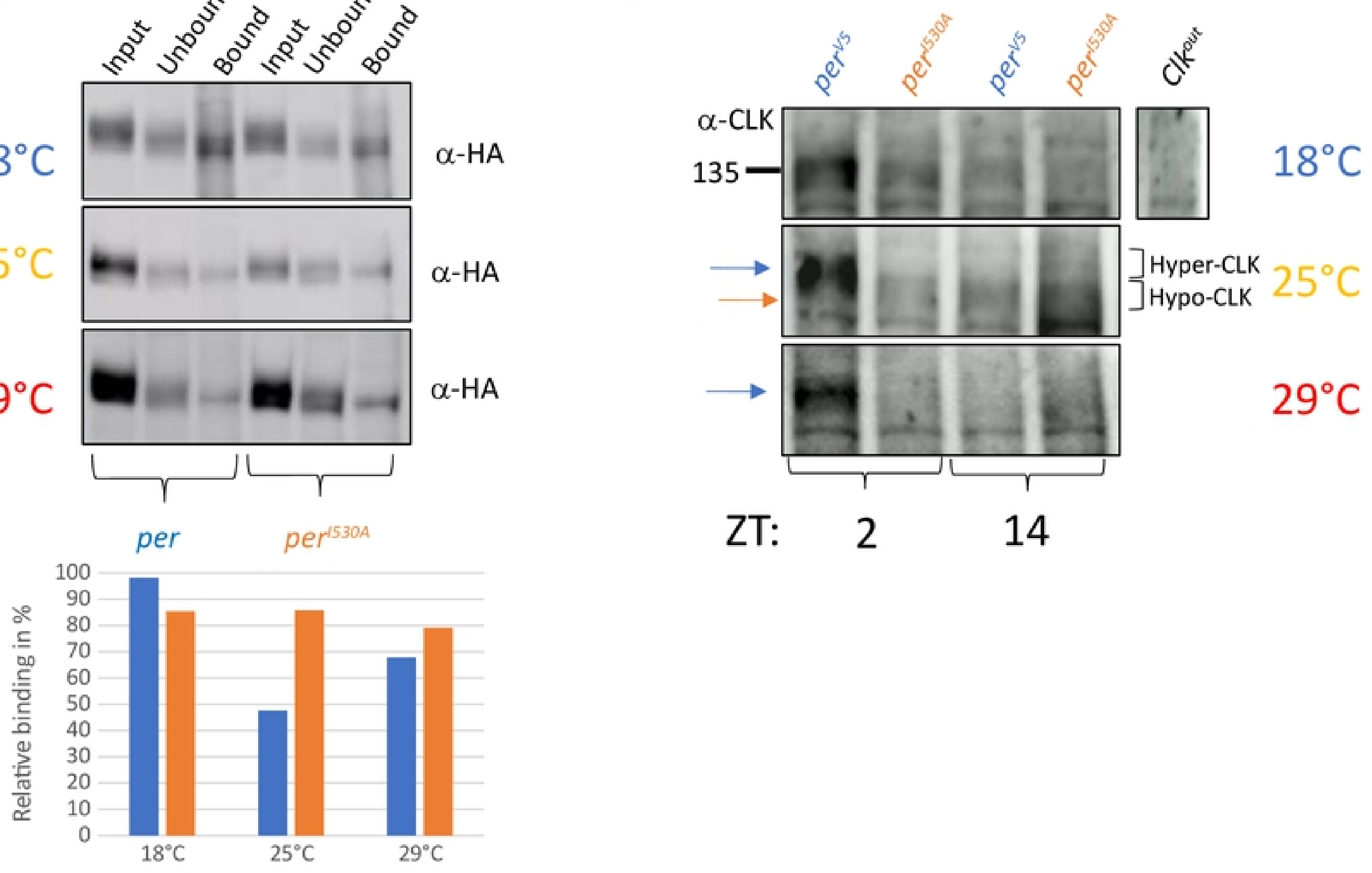
Figure 6
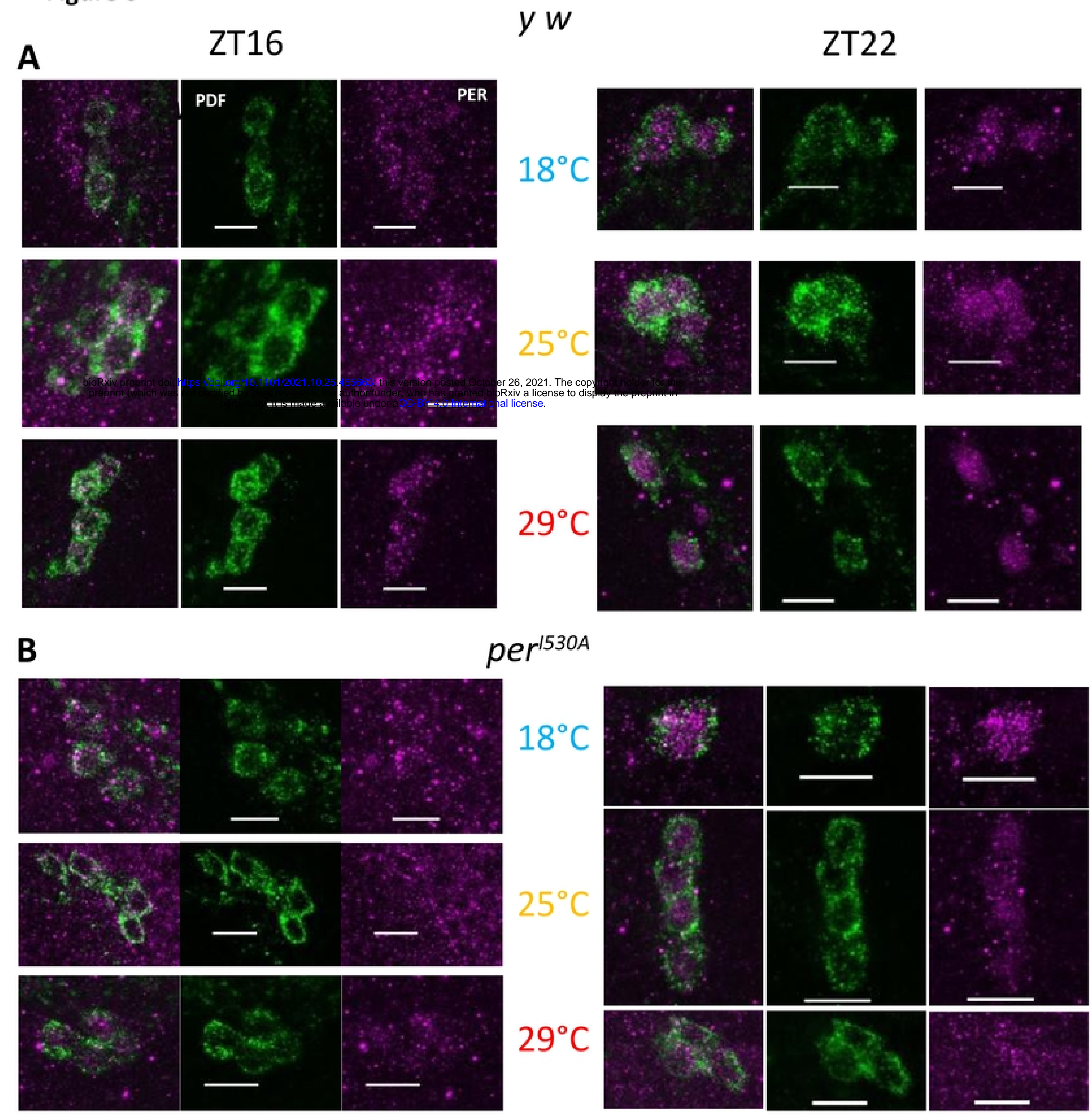

C
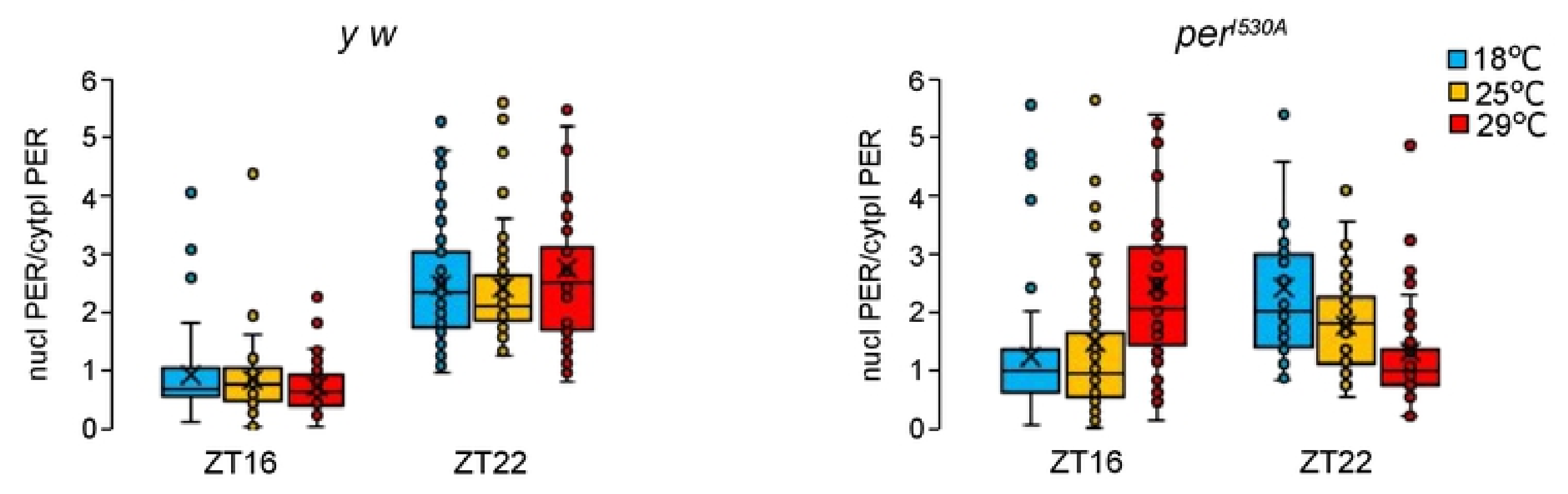
Figure S5

$y w$
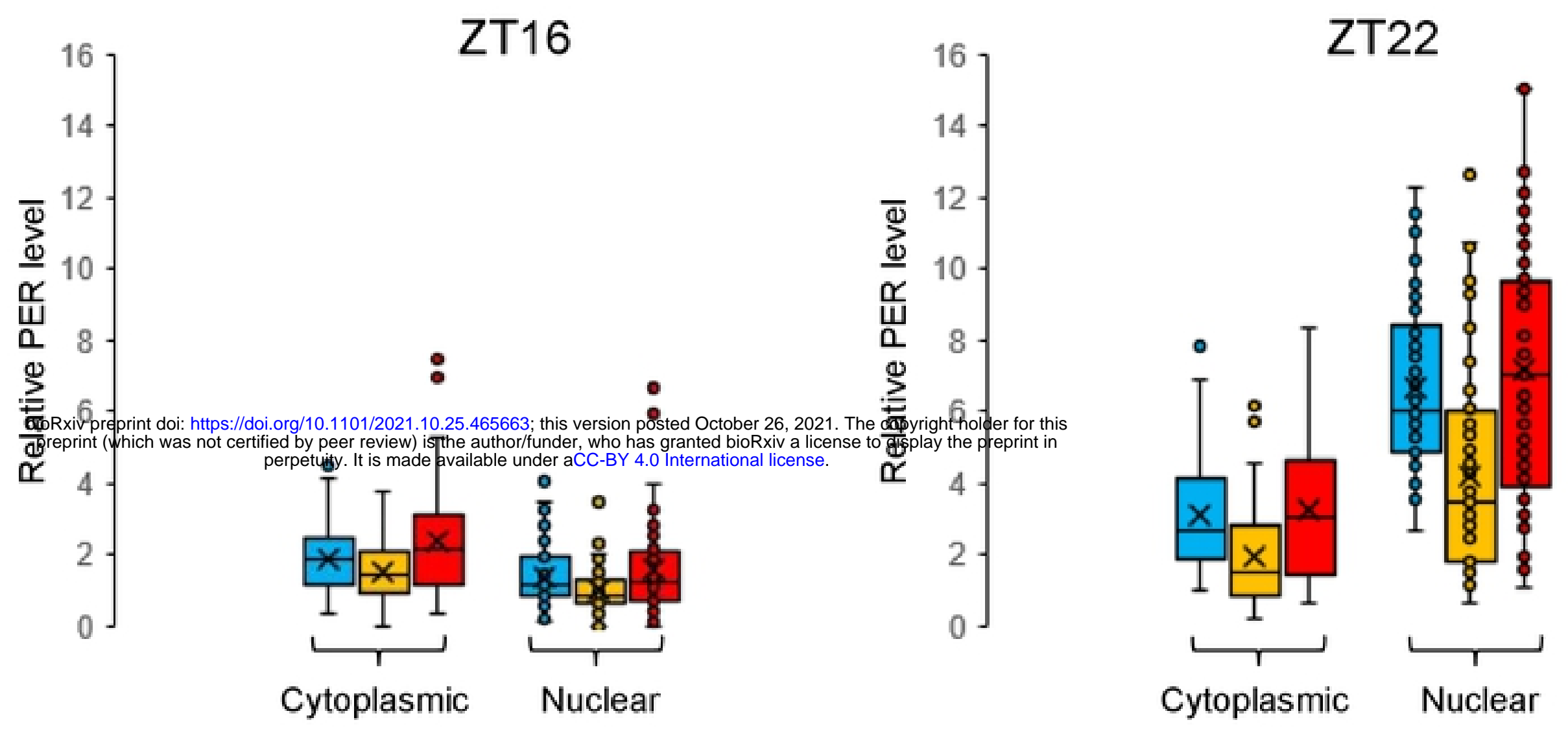

$\square 18^{\circ} \mathrm{C}$

$\square 25^{\circ} \mathrm{C}$

$29^{\circ} \mathrm{C}$

\section{per $r^{1530 A}$}
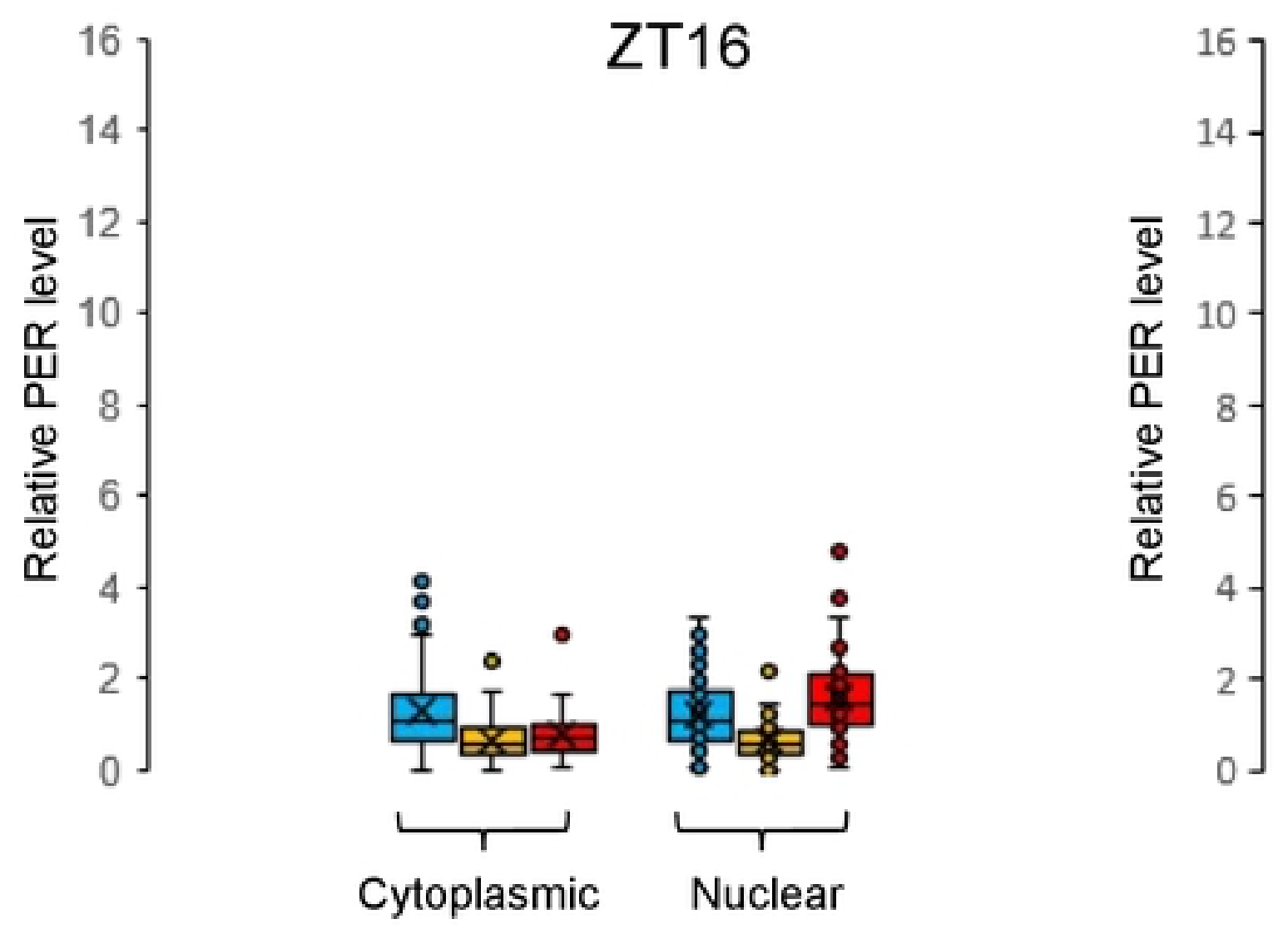

ZT22

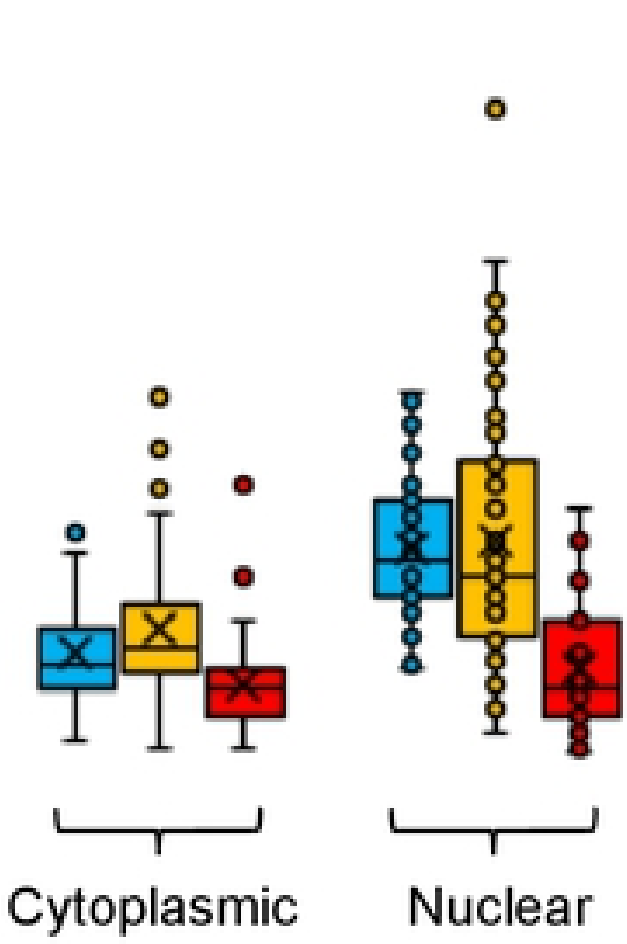




\section{Figure 7}

A
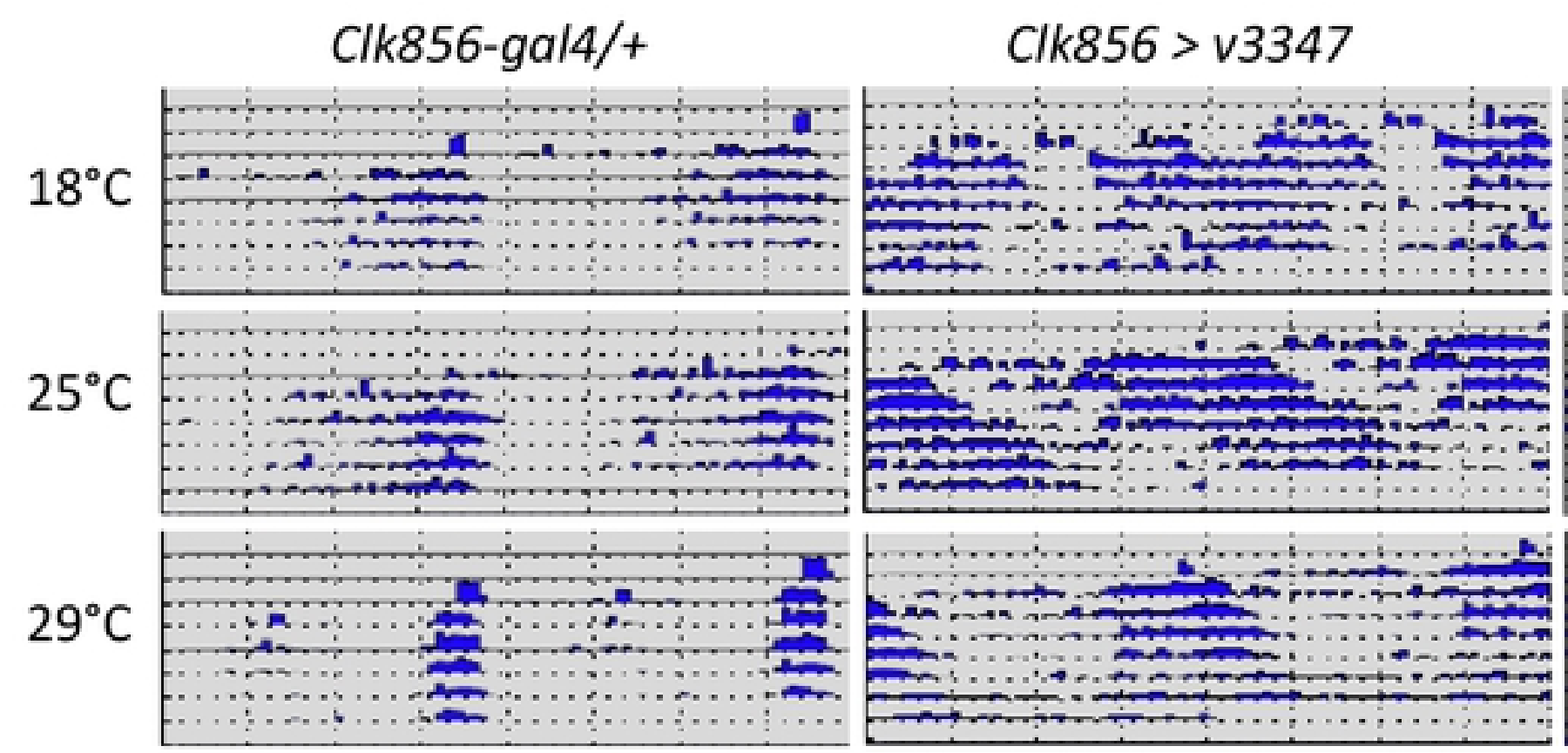

Clk856 > BL34021

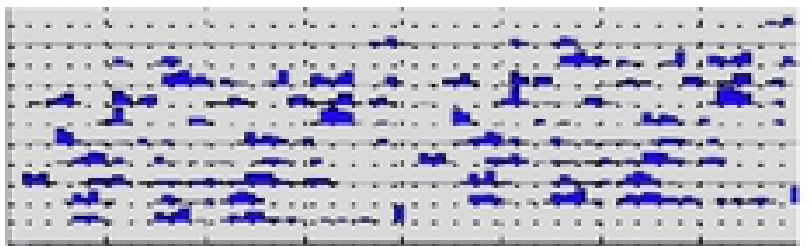

B

bioRxiv preprint doi: https://doi.org/10.1101/2021.10.25.465663; this version posted October 26, 2021. The copyright holder for this
preprint (which was not certified by peer review) is the author/funder, who has granted bioRxiv a license to display the preprint in

preprint (which was not certified by peer review) is the author/funder, who has granted bioRxiv a license
perpetuity. It is made available under aCC-BY 4.0 International license.
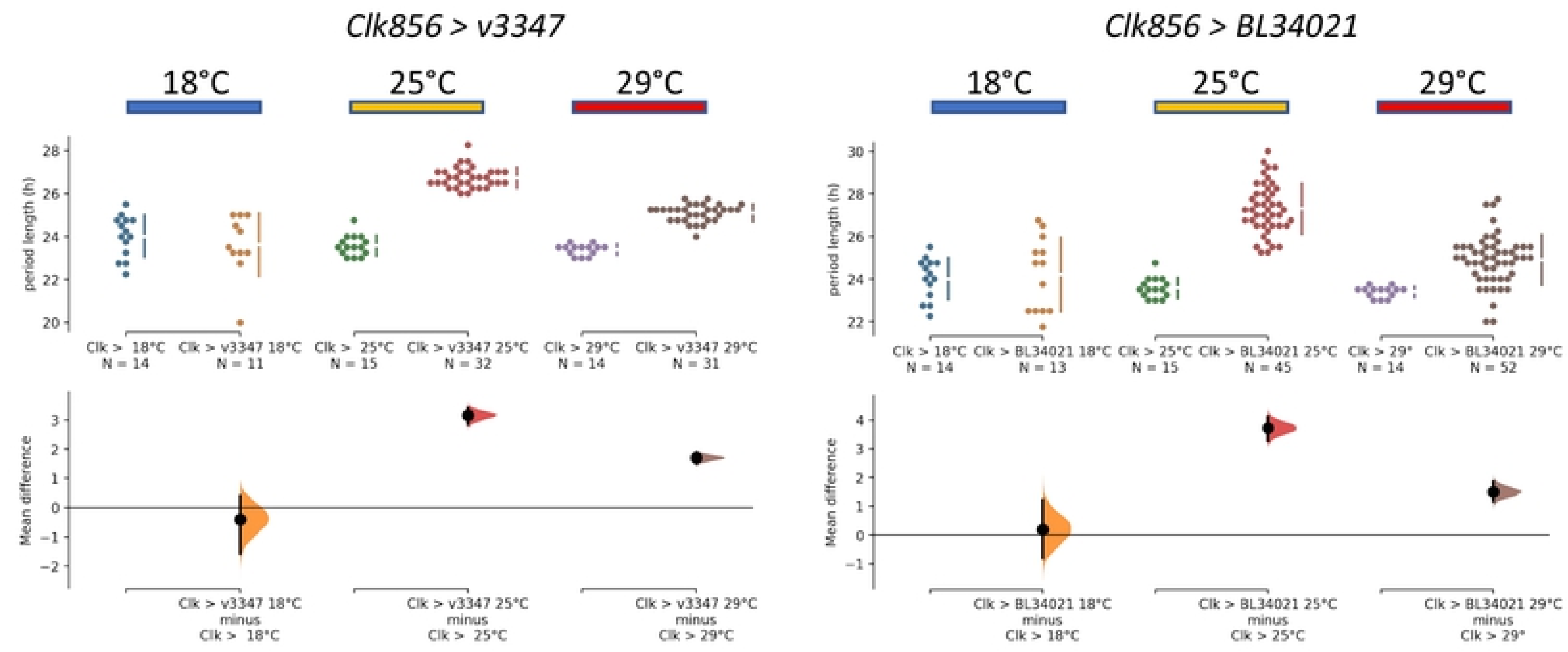

C
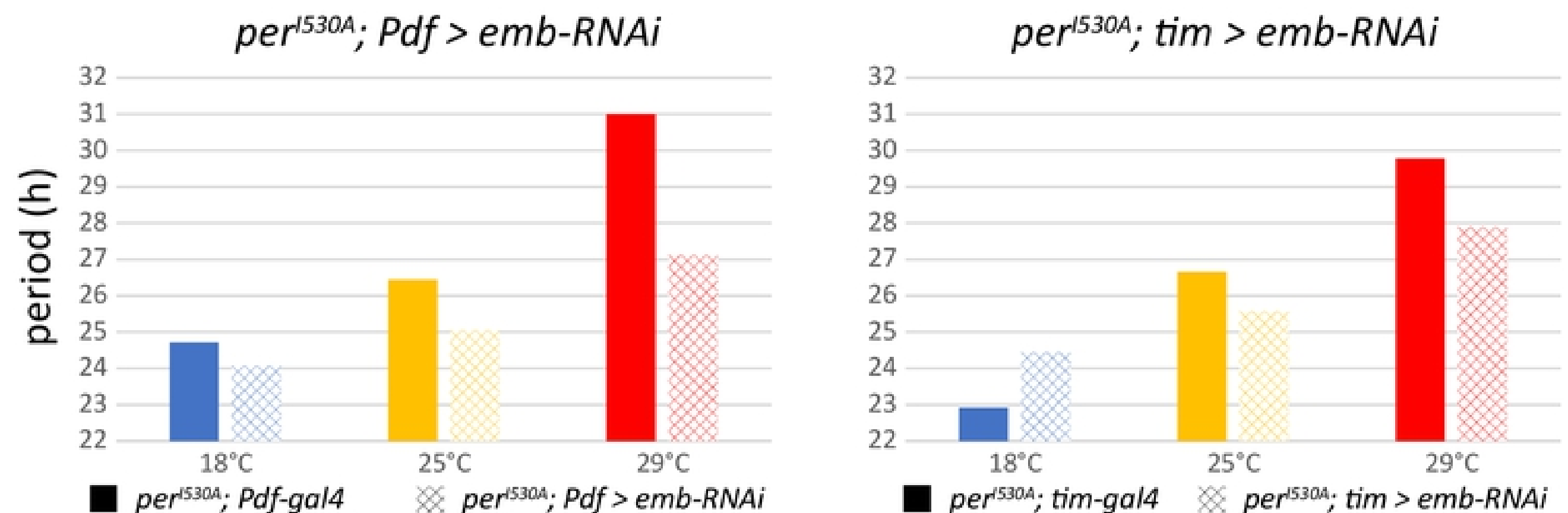